\title{
PHOTOINITIATED TRANSFORMATION OF NANOCRYSTAL SUPERLATTICE POLYMORPHS ASSEMBLED AT A FLUID INTERFACE
}

\author{
A Thesis \\ Presented to the Faculty of the Graduate School \\ of Cornell University \\ In Partial Fulfillment of the Requirements for the Degree of \\ Master of Science
}

by

Yingjie Gao

August 2020 
(C) 2020 Yingjie Gao 


\begin{abstract}
Spatial and temporal control the self-assembly of inorganic nanocrystals is both fundamentally interesting and technically challenging. Here, we apply photochemistry-based self-assembly to fabricate electronically coupled and locally ordered $\mathrm{PbS}$ quantum dots thin films at a liquid-air interface. We demonstrate the assembly process can be triggered by the activation of a photoacid generator, $\mathrm{Ph}_{2} \mathrm{I}-$ pyranine. The altered surface chemistry of the quantum dots allows for positive or negative pattern generation using quantum dots assemblies down to monolayer thickness. We show that the film structure can be tuned between six-fold and four-fold symmetry by controlling the exposure time. The influence of photo dosage on local assembly structures, optical properties and electronic conductivity is studied in details. We propose the photo-triggered strategy as a new approach to design nanocrystal assemblies for advanced fundamental studies and emerging technological applications.
\end{abstract}




\section{BIOGRAPHICAL SKETCH}

Yingjie Gao is from China. She got her Bachelor degree in Hunan University. There she joined in various research groups, built strong interest in continuing research work in Chemical Engineering. And she graduated as the Top student in the department because of her academic excellence and strong research ability.

Then she enrolled in the Master of science program in Cornell University in the Fall of 2018. Here, she joined Prof. Tobias Hanrath's Energy Lab and started her research project on Photoinitiated transformation of nanocrystal superlattice polymorphs assembled at a fluid interface. Her academic objective is to do advanced fundamental studies of nanoscience and develop her discoveries into technological applications. She will graduate with a M.S. in chemical engineering in June 2020. After graduation, she would move back to China and secure an R\&D position in nanotechnology. 


\section{ACKNOWLEDGMENTS}

Special thanks are due to my supervisor of the thesis, Prof. Tobias Hanrath, who gave me the chance to work on that particularly exciting research project and to benefit from his long experience in this field of nanoscience. Thanks Jen-Yu Huang for his outstanding support, instruction, inspiration, and advice which were essential to this thesis. Thanks Dr. Daniel Balazs for his expert knowledge of the field, recommendation of textbooks, and careful review of this thesis. Thanks for Yuanze $\mathrm{Xu}$ for his suggestions during my research work and completing this thesis. Thanks for the kindness help from all the group members in HELIOS's group.

And to my parents, Jianshe and Liqin, and brother, Jiajie, without their support, financially and mentally, this thesis would never have been completed. Thank them. 


\section{TABLE OF CONTENTS}

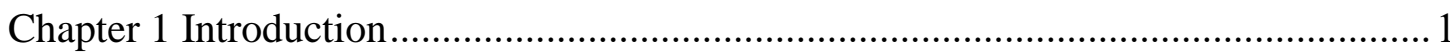

1.1 Building Blocks of Nanotechnology ........................................................... 1

1.2 Oriented attachment of nanoscale building blocks........................................... 3

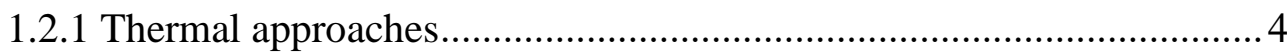

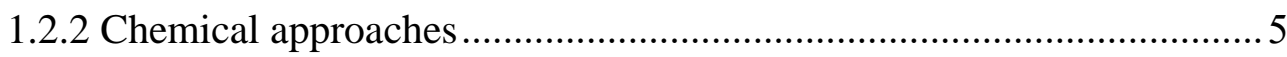

1.2.3 Additional methods ............................................................................

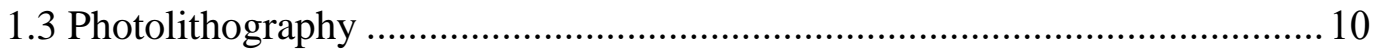

1.3.1 Direct optical lithography of functional inorganic nanomaterials....... 10

1.4 Nanoparticle self-assembly at fluid interfaces ............................................. 13

1.5 Optically-driven reorganization of colloidal assemblies ................................. 14

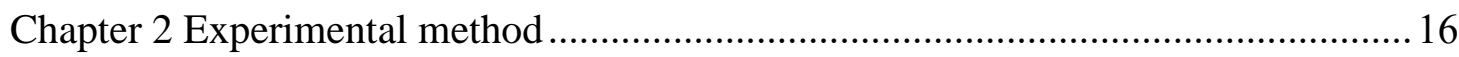

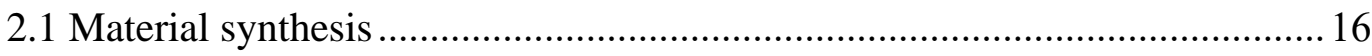

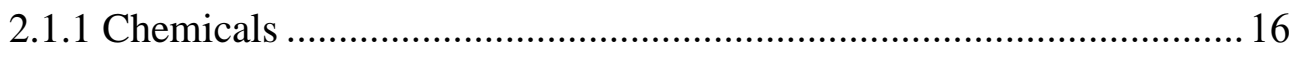

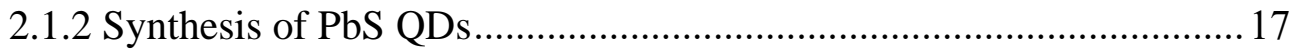

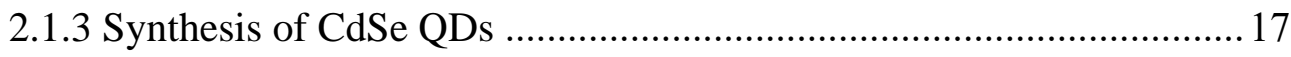

2.1.4 Synthesis of photoacid generator (PAG) ............................................ 18

2.2 Verification experiment of the chemistry ……………................................ 18

2.3 Design of experimental setup and experimental material conditions............. 19

2.4 Modify operation conditions for ORCA process..............................................21

2.4.1 Concentration range of photoacid generator (PAG) ............................ 21 
2.4.2 Concentration range of $\mathrm{PbS}$...........................................................22

2.4.3 Limiting thickness of PbS QDs ............................................................ 23

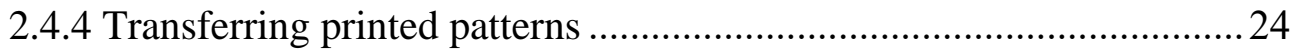

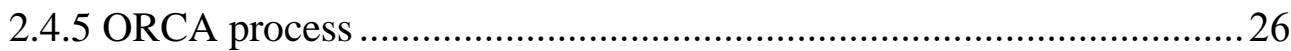

2.5 Characterization techniques and sample preparation ...................................22

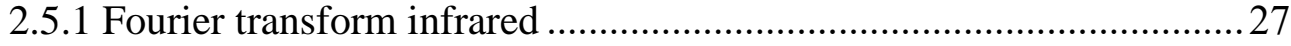

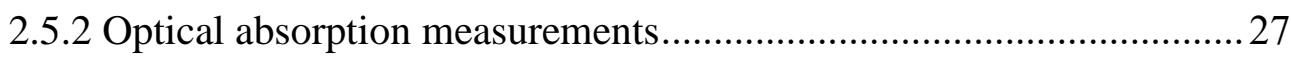

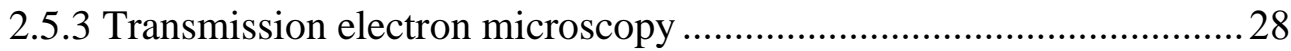

2.5.4 Two-terminal conductivity measurement.........................................29

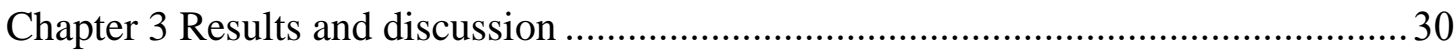

3.1 Reaction mechanism of ORCA process .......................................................... 30

3.1.1 Analysis of generation photolysis and ligand removal rate...................32

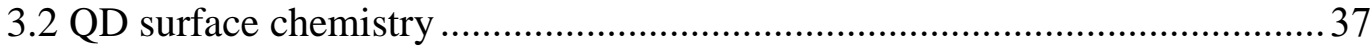

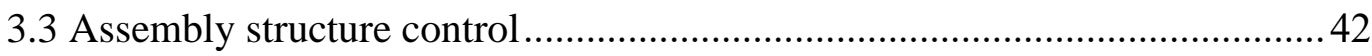

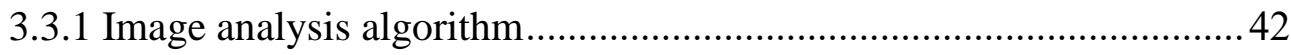

3.3.2 Assembly structure transformation...................................................... 43

3.3.3 Surface chemistry involved structure transformation............................ 45

3.4 QD size and interparticle spacing reduction..................................................4

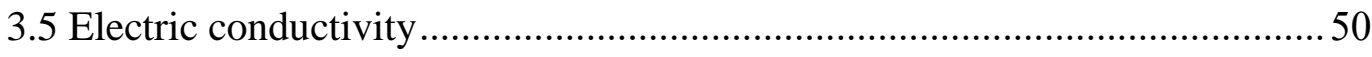

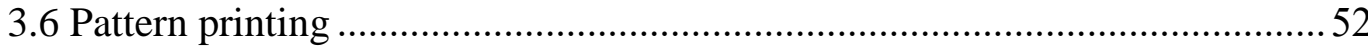

3.6.1 Positive pattern and negative pattern ..................................................52

3.6.2 Resolution of printed patterns ............................................................. 54

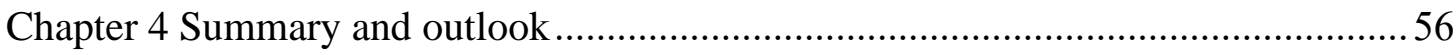




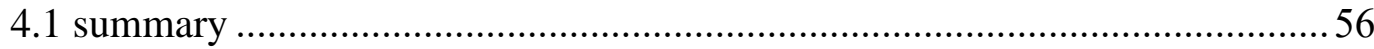

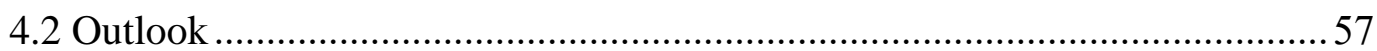

4.2.1 Improvement of printing resolution of ORCA process .....................57

4.2.2 Build photochemistry library for ORCA process .............................59

4.2.3 Layer-by-layer multicomponent of ORCA process ...........................6 60

4.2.4 ORCA process with two-photon lithography ................................6 61

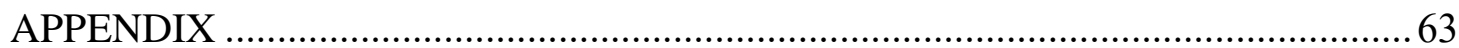

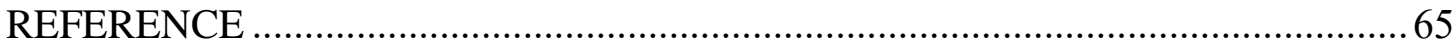




\section{LIST OF FIGURES}

Figure 1. Scheme illustrating the scale-range of nanoscience and two types of fabrication approaches. Some natural entities are included, for didactic

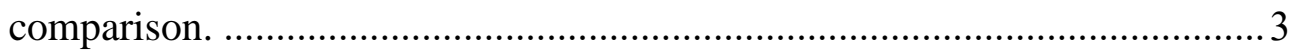

Figure 2. Influences of the reaction parameters on the structures formed by oriented attachment of PbSe QDs. ${ }^{16}$ a. Influence of the original particle concentration in the QD suspension. Reaction temperature: $50{ }^{\circ} \mathrm{C}$. The initial particle concentrations from left to right are: $7.0 \times 10^{-7}, 11.3 \times 10^{-7}$, $28 \times 10^{-7} \mathrm{~mol} / \mathrm{L}$. The insets show cartoons of the linear structures, honeycomb superlattices, and ultrathin sheets with square nanostructure. $b$. Influence of reaction temperature. The original particle concentration in the suspension was $14 \times 10^{-7} \mathrm{~mol} / \mathrm{L}$. The reaction temperature from left to right

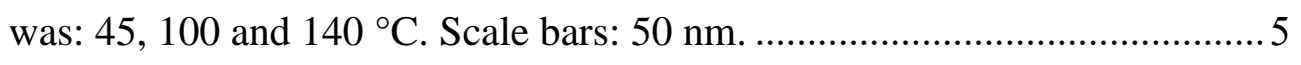

Figure 3. A schematic illustration of the experimental method. ${ }^{17}$....................... 6

Figure 4. Physicochemical processes at the liquid interface play a key role in the irreversible oriented attachment of adjacent QDs. ${ }^{12}$ a. Schematic representation of how the adding of ethylene diamine causing the formation and desorption of PbSe QD surface and fusing with a proximate particle. b. Representation of two particles at a fluid interface forming an epitaxial interdot bond. 7

Figure 5. Representation of the nanocrystal plasma polymerization process. ${ }^{18} \ldots .8$

Figure 6. Schematic illustration of the balance between quantum-confinement and quantum-coupling. ${ }^{20}$ a. Colloidal QDs passivated with organic ligands. b. 
Confined-but-connected structures. c. Complete coupling of the QDs leads

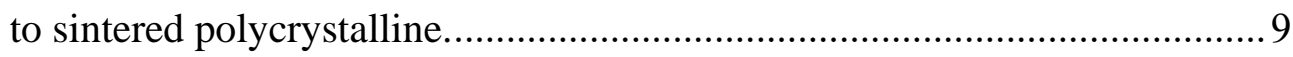

Figure 7. Schematic illustration of single scan laser spike annealing. ${ }^{21}$ a. Cartoon of single scan laser spike annealing fusing particles. b. Illustration of the three steps of QD fusion: ligand degradation, neck formation, and surface diffusion. c. PL spectra showing a quantized transition during the first QD fusion event. PL spectra are normalized by the total emission. The inset shows the change in particle diameter during the monomer to dimer fusion event. 9

Figure 8. Direct optical lithography of functional inorganic nanomaterials with photosensitive inorganic ligands. ${ }^{23}$ a. Schematic illustration of QDs with ion pair surface ligands. b. Two complementary approaches to design of photosensitive inorganic ligands. c. Different materials patterned by using $\mathrm{PAG}^{+}$-based ligands $\left(\mathrm{CdTe}, \mathrm{ZrO}_{2}, \mathrm{Fe}_{2} \mathrm{O}_{3}\right.$, and IGZO) and $\mathrm{CS}_{2} \mathrm{~N}_{3}{ }^{-}$ligands (CdSe, InP, Au, and $\mathrm{Al}_{2} \mathrm{O}_{3}$ ). Scale bars, $100 \mathrm{~mm}$ (left column); $5 \mathrm{~mm}$ (right column). (d) $\mathrm{CeO}_{2}$ nanocrystals patterned on a glass substrate by using $\left(\mathrm{Ph}_{2} \mathrm{I}\right)_{2} \mathrm{MoO}_{4}$ ligands. Scale bar, $5 \mathrm{~mm}$. e. (Top) Positive patterns of $\mathrm{CdSe} / \mathrm{ZnS}$ (red), InP/ZnS (green), and $\mathrm{ZnSe} / \mathrm{ZnS}$ (blue) core-shell QDs patterned with $\mathrm{NH}_{4} \mathrm{CS}_{2} \mathrm{~N}_{3}$ ligands. (Bottom) Negative patterns of oleatecapped $\mathrm{InP} / \mathrm{ZnS}$ (red), $\mathrm{CdSe} / \mathrm{ZnS}$ (green), and $\mathrm{ZnSe} / \mathrm{ZnS}$ (blue) core-shell QDs patterned by using $\left(\mathrm{p}-\mathrm{CH}_{3} \mathrm{~S}_{-}-\mathrm{C}_{6} \mathrm{H}_{4}\right)\left(\mathrm{C}_{6} \mathrm{H}_{5}\right)_{2} \mathrm{~S}^{+} \mathrm{OTf}^{-}$(OTf, triflate) PAG. Scale bars, $5 \mathrm{~mm}$ 12 
Figure 9. a. QD orientation (top) and multi-particle interactions at the fluid interface (middle) dictates the QD superstructure. TEM of square, line, and hexagonal structures. b. Directed assembly and attachment of QD at a liquid-liquid interface. Scale bars: 50nm (bottom). ${ }^{9}$................................ 14

Figure 10. UV-vis absorption spectrum of $\mathrm{Ph}_{2} \mathrm{I}$-pyranine.............................. 18

Figure 11. Spin coating results on silicon wafer. (A star, Letter A, Chinese character '远” and Cornell university logo were on the wafer).....

Figure 12. Sketch and procedure for ORCA process. a. Adding subphase $\left(\mathrm{Ph}_{2} \mathrm{I}-\right.$ pyranine in EG) and top phase (PbS capped with OA ligands in hexane). b. UV exposure after evaporation of hexane with covered quartz. c. Transferring. 20

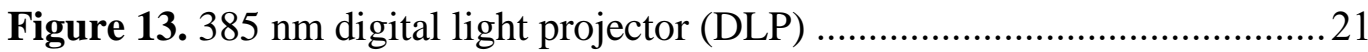

Figure 14. TEM image of $6.5 \mu \mathrm{L} 3 \mathrm{~g} / \mathrm{L} \mathrm{PbS}$ QDs in hexane evaperting on EG. Scale bar: $200 \mathrm{~nm}$. 23

Figure 15. Printed pattern (star) on wafer. a. 1 2 layers of PbS. b. $\sim 3$ layers of PbS. c. 4 5 layers of PbS. d. 6 layers of PbS. Scale bars: $1 \mathrm{~mm}$. 24

Figure 16. Comparison results of dry and wet cases. (a, d) Patterns after exposure, (b, e) After transferring to silicon wafer, (c, h) Patterns on silicon wafer. Scale bars: $5 \mathrm{~mm}$. 25

Figure 17. Comparison six kinds of substrates. a. HMDS functionalized wafer. b. MPTMS functionalized wafer. c. undoped silica wafer. d. wet thermal oxide

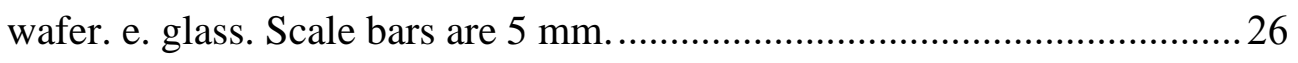

Figure 18. FTIR spectra for Pyranine and oleic acid.....................................2 27 
Figure 19. A schematic cross-sectional view of devices for measuring twoterminal conductivity 29

Figure 20. Mechanistic sequence of PAG photolysis and QD ligand removal of ORCA process. 32

Figure 21. Sequence of coupled sub-processes involved in ORCA processing. . 33

Figure 22. $\mathrm{PbS}$ QD model and surface area. 38

Figure 23. FTIR spectra of four different $\mathrm{PbS}$ thin films with different UV exposure time (Number shows the ligand coverage transformation). 39

Figure 24. Digital light processing enables local definition of the superlattice structure. (a) TEM image outside the exposed area. (b) photograph of the pattern transferred onto silicon wafer. (c) TEM image in the exposed area. Scale bars for (a) and (c) are $50 \mathrm{~nm}$. Scale bar for (b) is $1 \mathrm{~mm}$.

Figure 25. Symmetry transformation with different light dosage. The bottom left section of each panel shows the original TEM image and the rest of the panel shows the Voronoi cells color coded according to the local bond ordering parameter (blue scale of $\Psi_{6}$ if $\Psi_{6}>\Psi_{4}$ and red scale of $\Psi_{4}$ if $\Psi_{6}<$ $\Psi_{4}$ ). (a) $0 \mathrm{~s}$ with $8.9 \% \Psi_{4}$ symmetry. (b) $15 \mathrm{~s}$ with $26 \% \Psi_{4}$ symmetry. (c) 30 s with $60 \% \Psi_{4}$ symmetry. (d) $120 \mathrm{~s}$ with $59 \% \Psi_{4}$ symmetry. Scale bars for a$\mathrm{d}$ are $100 \mathrm{~nm}$. 44

Figure 26. Symmetry transformation with different PAG concentration. (blue scale of $\Psi_{6}$ if $\Psi_{6}>\Psi_{4}$ and red scale of $\Psi_{4}$ if $\Psi_{6}<\Psi_{4}$ ). (a) $0.25 \mathrm{~g} / \mathrm{L}$ with $28 \%$ $\Psi_{4}$ symmetry. (b) $0.5 \mathrm{~g} / \mathrm{L}$ with $60 \% \Psi_{4}$ symmetry. (c) $1 \mathrm{~g} / \mathrm{L}$ with $61 \% \Psi_{4}$ 
symmetry. (d) $2 \mathrm{~g} / \mathrm{L}$. Scale bars for a and b are $100 \mathrm{~nm}$, c and d are $50 \mathrm{~nm}$.

Figure 27. NIR absorption spectra.................................................................. 47

Figure 28. Comparison of QD size obtained from NIR measurements and TEM

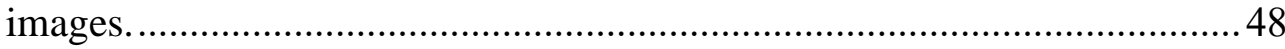

Figure 29. Radial distribution function analysis of TEM images......................... 49

Figure 30. ID-VD (output) characteristics of five devices..................................50

Figure 31. Resistivity of $\mathrm{PbS}$ thin films with different light dosage....................51

Figure 32. FTIR spectra of $\mathrm{PbS}$ film at $0 \mathrm{~s}$ and $60 \mathrm{~s}$ of $\mathrm{UV}$ exposure..................53

Figure 33. a. Negative pattern on silicon wafer. b. Positive pattern on silicon wafer. Scale bars: $1 \mathrm{~mm}$. 54

Figure 34. Printed pattern on silica wafer with different photodosage. a. 15 s. b. 30 s. c. 120 s. Bottom right section in a is the original exposed pattern for a, b and c. d-f are cooresponding enlarged views of a-c. Scale bars for a-c are 1 $\mathrm{mm}, \mathrm{d}-\mathrm{f}$ are $0.2 \mathrm{~mm}$ 55

Figure 35. Printed paterns on silica wafer with $\mathrm{PbS}$ or CdSe QDs. a. Star (CdSe). b. $\mathrm{PbS}(\mathrm{PbS})$. c. Checkerboard (PbS). 55

Figure 36. Printed star at the liquid surface after $30 \mathrm{~s}$ of UV exposure with different overall sitting time (Black dash line presents original printed edge and yellow dash line presents extended edge after certain siting time). a. 3 mins. b. 5 mins. c. 7 mins. Scale bars for a-c are $1 \mathrm{~mm}$..............................58

Figure 37. Diffusion condition on the liquid surface..........................................59 
Figure 38. Chemical Structures of Designed Photoactive Ligands Used for DOLFIN $^{1}$ 60

Figure 39. Experimental process of LbL assembly of ORCA. a. Adding subphase and top phase. b. UV exposure with covered quartz. c. Transferring. d. LbL atachment 61

Figure 40. Process for 3D printing of inorganic nanocrystals. 62

Figure S1. FTIR spectra of PbS QD thin films after 120 s of UV exposure and $\mathrm{Ph}_{2} \mathrm{I}$-pyranine 63

Figure S2. Negative pattern of a thick PbS QD film. a. Negative pattern on silica wafer. b. Negative pattern under microscope. c. Large version of film in the yellow dash-line box. d. Large version of film in the yellow dash-line box. Darker areas are empty. Scale bars: $\mathrm{a}$ and b are $1 \mathrm{~mm}, \mathrm{c}$ is $0.1 \mathrm{~mm}$ and $\mathrm{d}$ is $0.05 \mathrm{~mm}$ 63

Figure S3. Radial distribution function analysis of TEM images. 64 


\section{LIST OF TABLES}

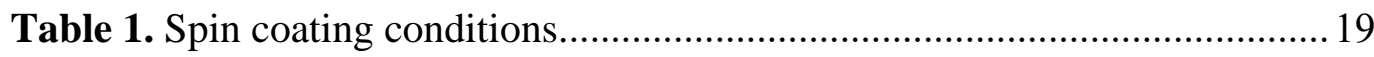

Table 2. Experimental results for different $\mathrm{Ph}_{2} \mathrm{I}$-pyranine concentration. (Adding $1 \mathrm{ml}$ subphase $20 \mu 11.01 \mathrm{~g} / \mathrm{l} \mathrm{PbS}$ (in toluene), observing results after 2 mins

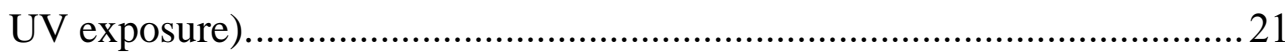

Table 3. Printed conditions for each case. ........................................................ 23

Table 4. Ligand coverage after certain dosage of UV exposure ....................... 40 


\section{LIST OF ABBREVIATIONS}

\begin{tabular}{|c|c|}
\hline QDs & Quantum dots \\
\hline QLEDs & QDs-based light emitting diodes \\
\hline EG & Ethylene glycol \\
\hline NPP & Nanocrystal plasma polymerization \\
\hline PL & Photoluminescence \\
\hline DOLFIN & $\begin{array}{l}\text { Direct optical lithography of } \\
\text { functional inorganic nanomaterials }\end{array}$ \\
\hline FTIR & Fourier transform infrared \\
\hline SEM & Scanning Electron Microscope \\
\hline TEM & Transmission electron microscopy \\
\hline OA & Oleic acid \\
\hline DLP & Digital light projector \\
\hline ODE & 1-octadecene \\
\hline$(\mathrm{TMS})_{2} \mathrm{~S}$ & Bis(trimethylsilylmethyl) sulfide \\
\hline ORCA & $\begin{array}{c}\text { Optically-driven reorganization of } \\
\text { colloidal assemblies }\end{array}$ \\
\hline PAG & Photoacid generator \\
\hline MPTMS & (3-mercaptopropyl) trimethoxysilane \\
\hline HMDS & Hexamethyldisilazane \\
\hline NIR & Near infrared \\
\hline RDF & radial distribution function \\
\hline
\end{tabular}


$\mathrm{LbL}$

Layer-by-layer

TPL

Two-photon lithography 


\section{Chapter 1 Introduction}

This chapter is devoted to explaining the motivation for the study of photoinitiated transformation of nanocrystal superlattice polymorphs assembled at a fluid interface in this thesis. The concepts of building block of nanotechnology, semiconductor nanocrystals and quantum dots (QDs) are introduced. And the current approaches to create superstructures of these materials in which constituent dots can purposefully interact are discussed, setting out the interesting challenge from a fundamental scientific perspective since there are many predictions of new emerging properties. A broad description of the nanostructures studied in this thesis and the main objectives of the study are shown. At last, the perspective application fields in the future to extend this project are demonstrated.

\subsection{Building Blocks of Nanotechnology}

Nanotechnology is a field precisely manipulating matter on an atomic, molecular, and supramolecular scale to fabricate functional new materials and novel nanoscale devices. ${ }^{1}$ Nanocrystals that are usually regarded as "artificial atoms" are the nanoscale 
building blocks for the fabrication. The nano-building blocks can be classified by dimensionalities: zero-dimensional (0D) quantum dots (QDs) including spheres, cubes, and tetrahedrons, one-dimensional (1D) nanorods and wires, two-dimensional (2D) nanodiscs and plates, and other advanced shapes such as rod-based multipods and nanostars. ${ }^{2}$ Zero-dimensional nanocrystals, QDs, are one of the biggest success in nanotechnology. They are a central topic in nanotechnology after they were discovered in 1980 by Russian physicist Alexei Ekimov. QDs are semiconductor nanocrystals composed of groups II to VI or III to V elements and have properties intermediate between bulk materials and discrete atoms or molecules. They have discrete energy levels and the band gaps can be tuned by size. ${ }^{3}$ Thus they exhibit size-dependent optical and electronic properties. ${ }^{4}$ Generally, there are two approaches to synthesize QDs, top-down processing and bottom-up methods as shown in Figure 1. Top-down methods is more familiar to engineers. It carves out materials lithographically or electrochemically from a semiconductor substrate such as e-beam lithography, and Xray lithography. ${ }^{5}$ Bottom-up approach, preparing QDs in solution as colloids by reacting precursors together, is more familiar to chemists. ${ }^{5}$ QDs are widely used in various fields of technology, such as electroluminescence device, QDs-based light emitting diodes (QLEDs) and solar cells. ${ }^{6}$ They also have biological applications such as fluorescent labelling of cellular proteins and cell tracking. ${ }^{7}$ Recent advances in the availability of nanostructured materials with controlled size, shape and composition have created an exciting new opportunity space to create novel materials and enabled applications. We're inspired and intrigued by the broad prospects of creating complex hierarchical multifunctional structures by leveraging concurrent advances 
nanofabrication methods and the availability of increasingly sophisticated nanomaterial-derived building blocks.

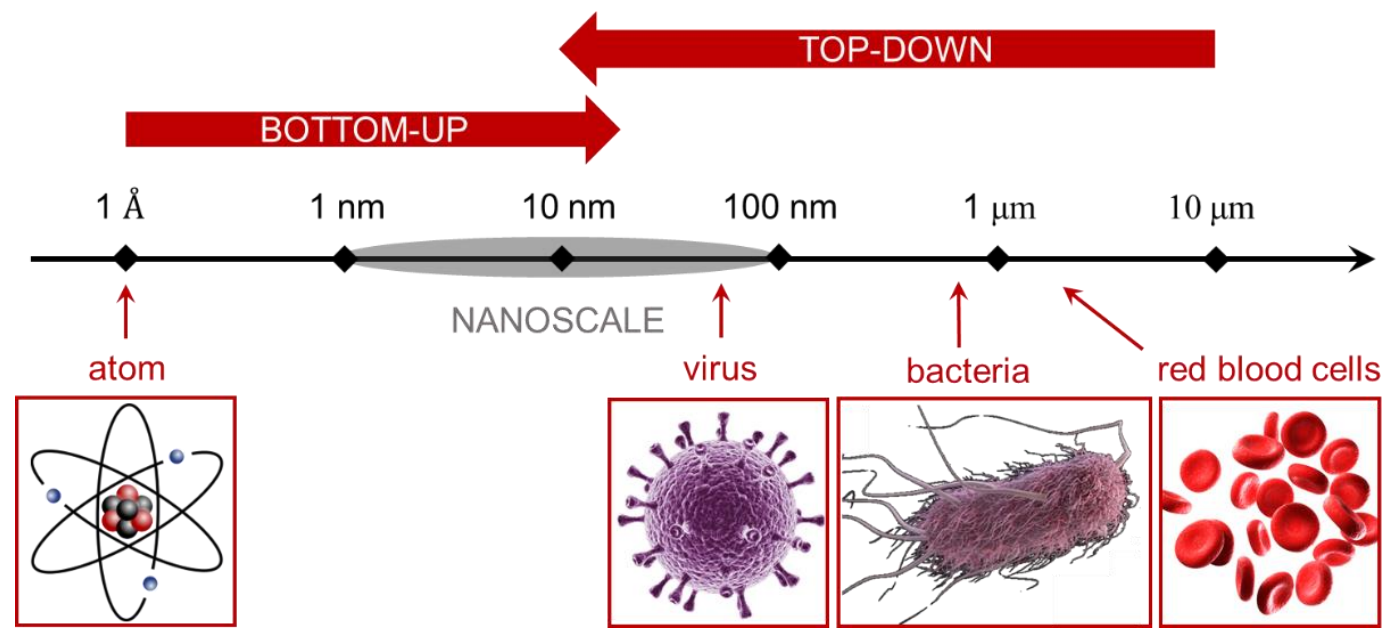

Figure 1. Scheme illustrating the scale-range of nanoscience and two types of

fabrication approaches. Some natural entities are included, for didactic comparison.

\subsection{Oriented attachment of nanoscale building blocks}

Nanofabrication is an ensemble method to fabricate different kinds of individual building blocks into hierarchic systems. New functional materials and novel devices can be created by different kinds of nanofabrication methods. Oriented attachment of QDs has generated significant interest recently as a nanofabrication method to connect individual building blocks via atomic matching of opposing crystal facets. Oriented attachment of QDs is different from QD self-assembly in the strength of the driving forces. The former involves interatomic bonding but the latter case involves van der Waals interactions or entropic factors. ${ }^{8}$ During oriented attachment, the internanocrystalline connection achieved through the "necking process"9-11 is indispensable for adjusting the coherent coupling effect in the superlattice, ${ }^{12}$ and thus can achieve 
various novel properties. ${ }^{12-14}$ For instance, epitaxially connected quantum dots combine size-quantization effects with high charge carrier mobilities which would lead to highly performing QD devices. To date, several strategies covering physical and chemical procedures have been adopted, such as thermal and chemical treatment.

\subsubsection{Thermal approaches}

Cho et al. reported the formation of nearly defect-free crystal lattice and high uniformity of micrometer-long $\mathrm{PbSe}$ wires at elevated temperature, formed by the consecutive fusing of PbSe nanocrystals along identical crystal faces. ${ }^{15}$ The entire process of nanowire formation is within one minute after the start of reaction and easy for solution-processing. The essential factors of nanowire formation are the controlling of concentration of the reactants, reaction temperature, and concentration and composition of stabilizing agents they used during the reaction. Besides the formation of 1D nanowire, Evers et al. obtained $1 \mathrm{D}$ and 2D PbSe single crystals by control of the temperature and particle concentration. ${ }^{16}$ They oriented attached of PbSe QDs in a thin film casted on an immiscible liquid and varied reaction concentration and temperature to vary symmetry of attachment from linear to $2 \mathrm{D}$ and even to $3 \mathrm{D}$ as shown in Figure 2. Figure $2 \mathrm{a}$ presents that by simply increasing the particle concentration, the QD attachment developed from nanocrystal wires to a $2 \mathrm{D}$ honeycomb lattice and finally to a $2 \mathrm{D}$ square lattice. Figure $2 \mathrm{~b}$ shows the formation of square $2 \mathrm{D}$ lattice at lower temperature $\left(45^{\circ} \mathrm{C}\right)$ and formation of $1 \mathrm{D}$ nanorods at higher temperatures as the particle concentration is sufficiently high. Thermal approaches present promising ability to epitaxially connected quantum dots. However, thermal treatments may cause the loss of single particle characteristics and consequently attenuate quantum 
confinement.

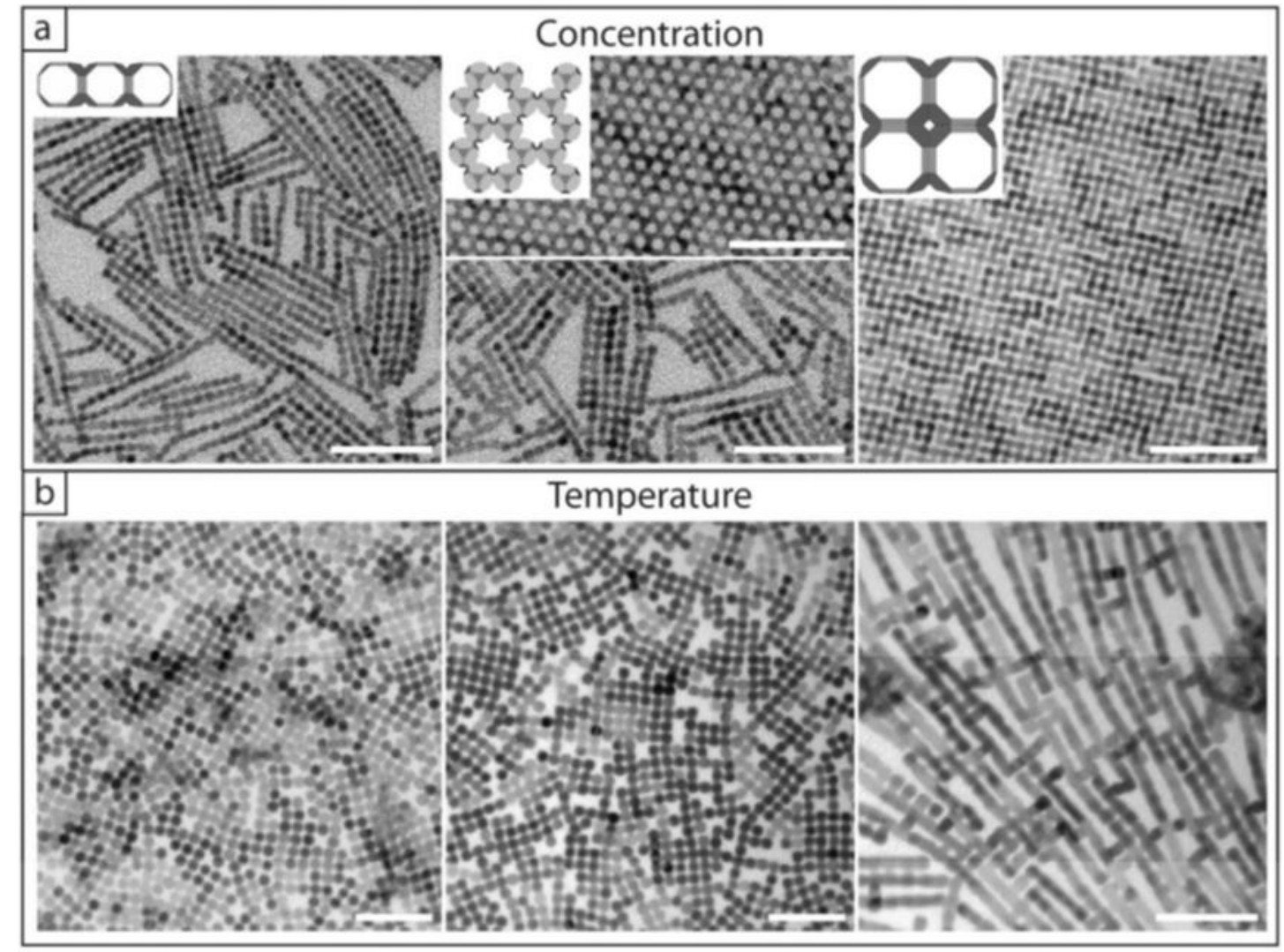

Figure 2. Influences of the reaction parameters on the structures formed by oriented

attachment of PbSe QDs. ${ }^{16}$ a. Influence of the original particle concentration in the QD suspension. Reaction temperature: $50^{\circ} \mathrm{C}$. The initial particle concentrations from left to right are: $7.0 \times 10^{-7}, 11.3 \times 10^{-7}, 28 \times 10^{-7} \mathrm{~mol} / \mathrm{L}$. The insets show cartoons of the

linear structures, honeycomb superlattices, and ultrathin sheets with square nanostructure. b. Influence of reaction temperature. The original particle concentration in the suspension was $14 \times 10^{-7} \mathrm{~mol} / \mathrm{L}$. The reaction temperature from left to right was: 45, 100 and $140{ }^{\circ} \mathrm{C}$. Scale bars: $50 \mathrm{~nm}$.

\subsubsection{Chemical approaches}

Using chemical triggers to remove initial surface binding ligands is another highly 
used approach recently to form epitaxially connected QD lattice. Walravens et al. reported chalcogen-adding and lead oleate displacing additives induced small area epitaxial superlattices of $\mathrm{PbSe}$ quantum dots with two kinds of chemical triggers, sodium sulfide and amines, respectively. ${ }^{17}$ Figure 3 demonstrates the experimental method used in their work. After spreading a QD monolayer on ethylene glycol (EG), the chemical trigger sodium sulfide or amines is injected. This is seen visually as a contraction and darkening of the QD monolayer in Figure 3. The insets cartoons show the structure transformation before and after injection from honeycomb superlattices to square nanostructure.

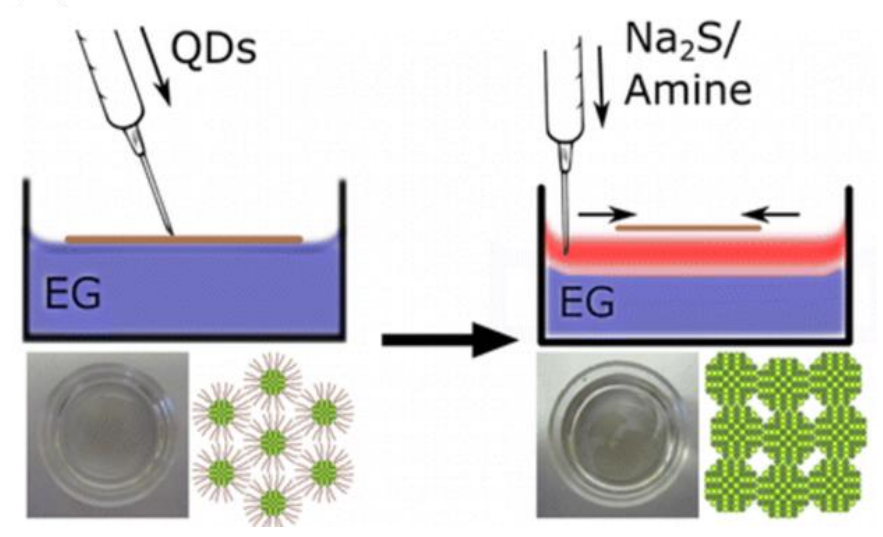

Figure 3. A schematic illustration of the experimental method. ${ }^{17}$

Whitham and Prof. Hanrath used ethylene diamine as a chemical trigger to deprotect PbSe QD surface and fuse the particles as displayed in Figure $4 .^{12}$ They also studied the basic mechanism of formation of epitaxially connected QD solids in the context of a coherent phase transition with distinct nucleation and propagation steps. 

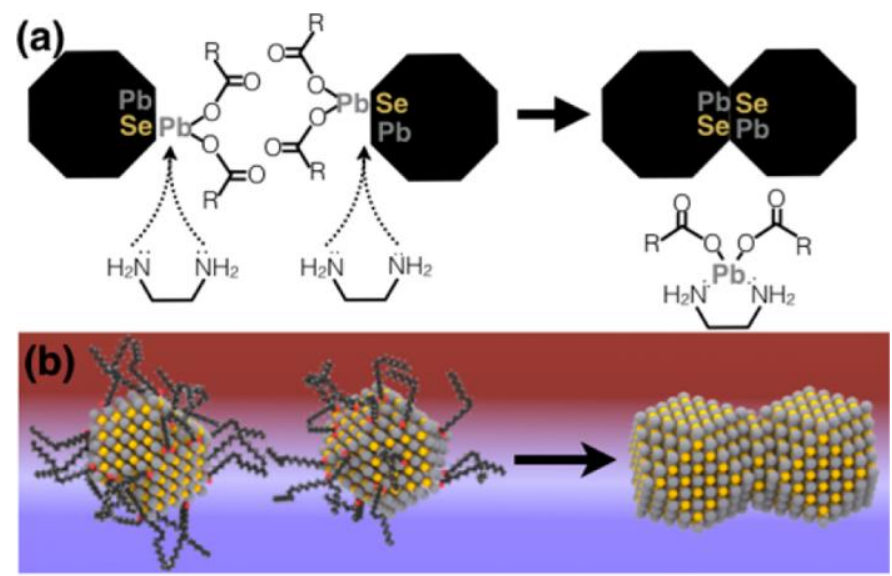

Figure 4. Physicochemical processes at the liquid interface play a key role in the irreversible oriented attachment of adjacent QDs. ${ }^{12}$ a. Schematic representation of how the adding of ethylene diamine causing the formation and desorption of $\mathrm{PbSe} \mathrm{QD}$ surface and fusing with a proximate particle. $b$. Representation of two particles at a fluid interface forming an epitaxial interdot bond.

\subsubsection{Additional methods}

Additional methods employed to interconnect QDs include plasma processing and pulsed laser annealing. Cademartiri et al. studied nanocrystal plasma polymerization to assemble QDs through an inorganic matrix as shown in Figure 5. ${ }^{18}$ Nanocrystal plasma polymerization (NPP) is a plasma treatment of nanostructure assemblies, preserving the size-dependent properties and individuality while consolidating them within a host matrix. NPP enabled the formation of free-standing $1 \mathrm{D}, 2 \mathrm{D}$, and $3 \mathrm{D}$ architectures composed of QDs. 


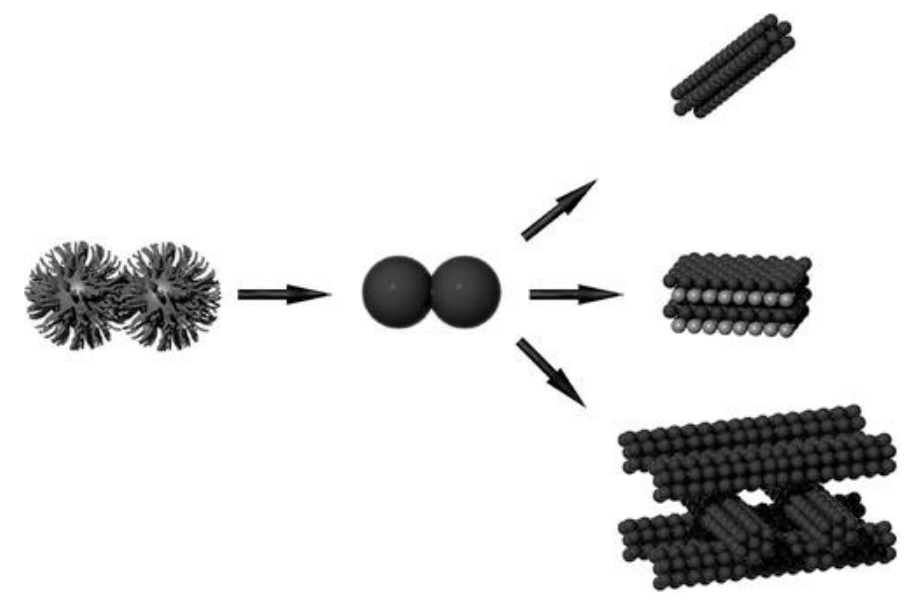

Figure 5. Representation of the nanocrystal plasma polymerization process. ${ }^{18}$

Laser annealing shows plenty advantages in the fabrication of semiconductor thin films. ${ }^{19}$ Baumgardner et al. applied pulsed laser annealing to process QD thin films into electrically coupled nanostructures. ${ }^{20}$ Figure 6a shows as-synthesis QDs coated with long-chain organic ligands passivated the QD surfaces. Figure $6 \mathrm{~b}$ presents confined-but-connected structures that balance quantum confinement and coupling. Figure 1.6c shows the completed coupling QD solids after pulsed laser annealing. After this work, Baumgardner et al. employed single scan laser spike annealing on CdSe QD thin films as an experimental test bed to demonstrate how the sizedependent photoluminescence (PL) emission can be tuned throughout the visible range and in spatially defined profiles during a single annealing step. ${ }^{21}$ The process involved three steps as displayed in Figure 7b, ligand degradation, neck formation, and surface diffusion to form a fully fused dimer. The PL spectra in Figure 7c indicates the change in particle diameter during the monomer to dimer fusion event. 

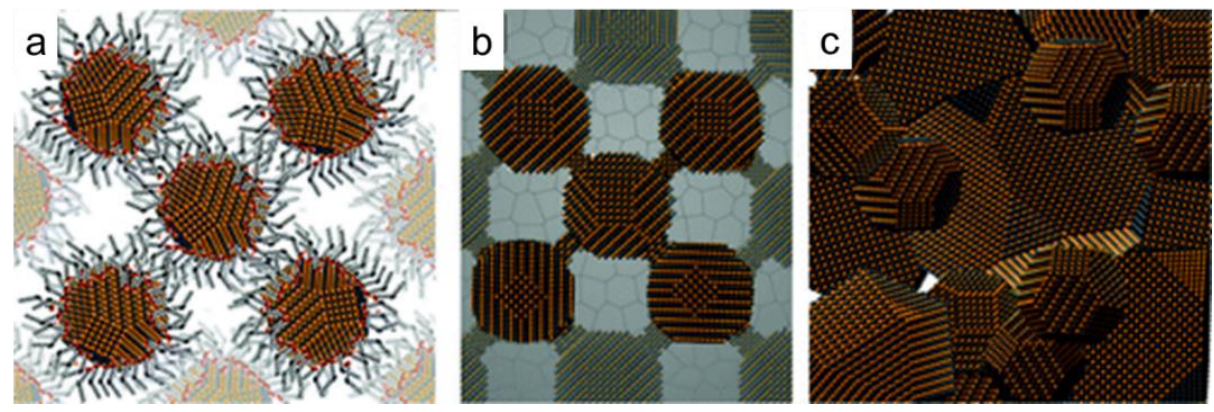

Figure 6. Schematic illustration of the balance between quantum-confinement and quantum-coupling. ${ }^{20}$ a. Colloidal QDs passivated with organic ligands. b. Confinedbut-connected structures. c. Complete coupling of the QDs leads to sintered polycrystalline.
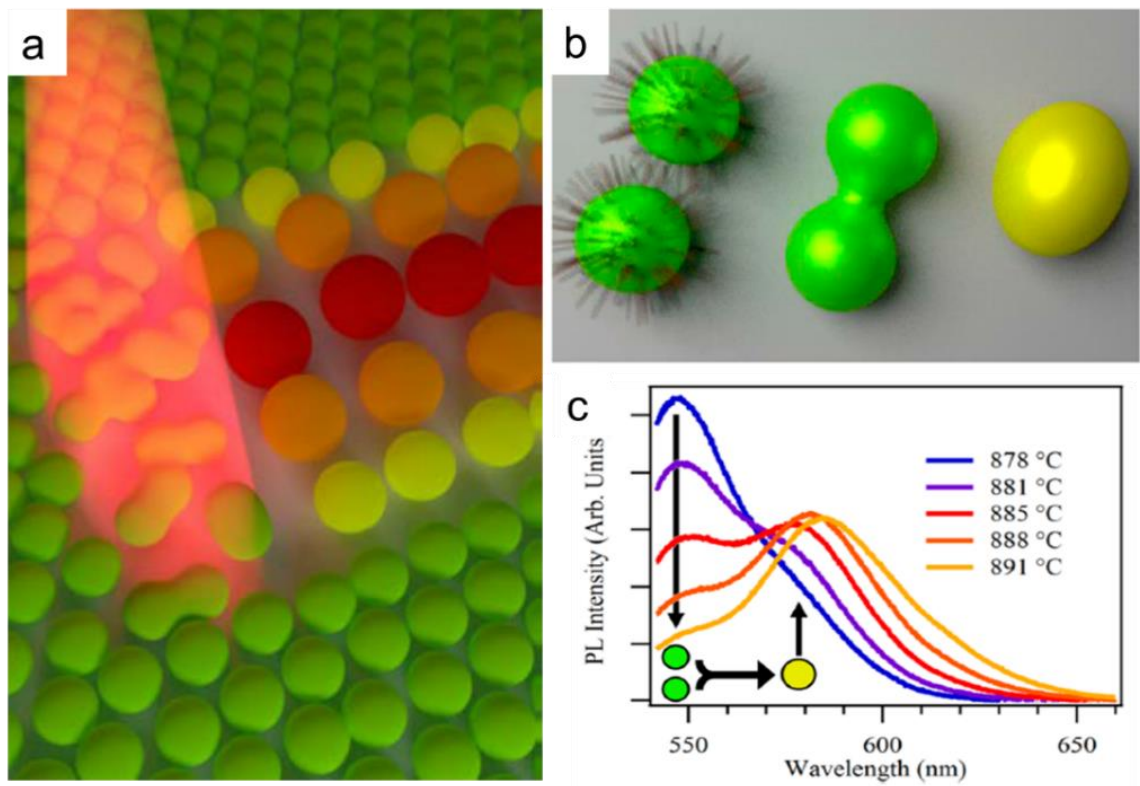

Figure 7. Schematic illustration of single scan laser spike annealing. ${ }^{21}$ a. Cartoon of single scan laser spike annealing fusing particles. b. Illustration of the three steps of QD fusion: ligand degradation, neck formation, and surface diffusion. c. PL spectra showing a quantized transition during the first QD fusion event. PL spectra are normalized by the total emission. The inset shows the change in particle diameter during the monomer to dimer fusion event. 


\subsection{Photolithography}

Within current approaches discussed above, the complex transport processes involved in the initiation and subsequent transformation presents several challenges limiting the formation of high-fidelity assemblies. ${ }^{22}$ There remains a large gap between locally controlling the formation of epitaxial connections and the rational fabrication of large scale, 2D superlattices usable for applications. To advance beyond thermal and chemical triggers, we hypothesized that adopting photolithographic patterning concepts provide an opportunity to achieve spatial and temporal control over the transformation. Photolithography, also called UV lithography, is a important process used in microfabrication to pattern parts on a thin film or the bulk of a substrate. Conventionally, photolithography uses light to transfer a geometric pattern from a photomask to a photosensitive chemical photoresist on the substrate. Photoresists, typically polymer formulations, would change solubility when illuminated with ultraviolet light. However, the application is restricted because of the reliance on photoresist during the fabrication process.

\subsubsection{Direct optical lithography of functional inorganic nanomaterials}

Yuan et al. demonstrated a method called direct optical lithography of functional inorganic nanomaterials (DOLFIN). ${ }^{23}$ This process combines multiple benefits of traditional photolithography and is designed toward efficient patterning of inorganic nanocrystals without diluting or contaminating them with organic photoresists and other by-products. To implement DOLFIN, they designed a library of QDs ion pair surface ligands, photoactivated $\mathrm{X}^{-}$and $\mathrm{Cat}^{+}$groups as displayed in Figure 8b. The photoactive anionic or cationic ligands show strong UV absorption bands and alter QD 
solubility in polar and nonpolar solvents when illuminated with UV light. Patterns can be created by illuminating QD films through a mask and then washing off the unexposed QDs. Figure 1.8c-e shows various patterns created by different kinds of QD materials with different kinds of ligands. In their later work, they extended the versatility of DOLFIN by designing a series of photoactivated QD surface ligands for direct patterning using various photon energies including DUV, near-UV (i-line, 365 $\mathrm{nm}$ ), blue (h-line, $405 \mathrm{~nm}$ ), and visible (450 nm) light. The DOLFIN approach, relying on photo-released chemicals to locally trigger solubility change of QDs thin films on solid substrates, has created new prospects to spatially pattern QD assemblies. Although promising, the reported methods focus on micron-scale pattern generation based on disordered arrays of QDs, and do not take guided self-assembly and particle ordering into consideration. There remains a large knowledge gap and huge potential in connecting photochemistry with self-assembly. 

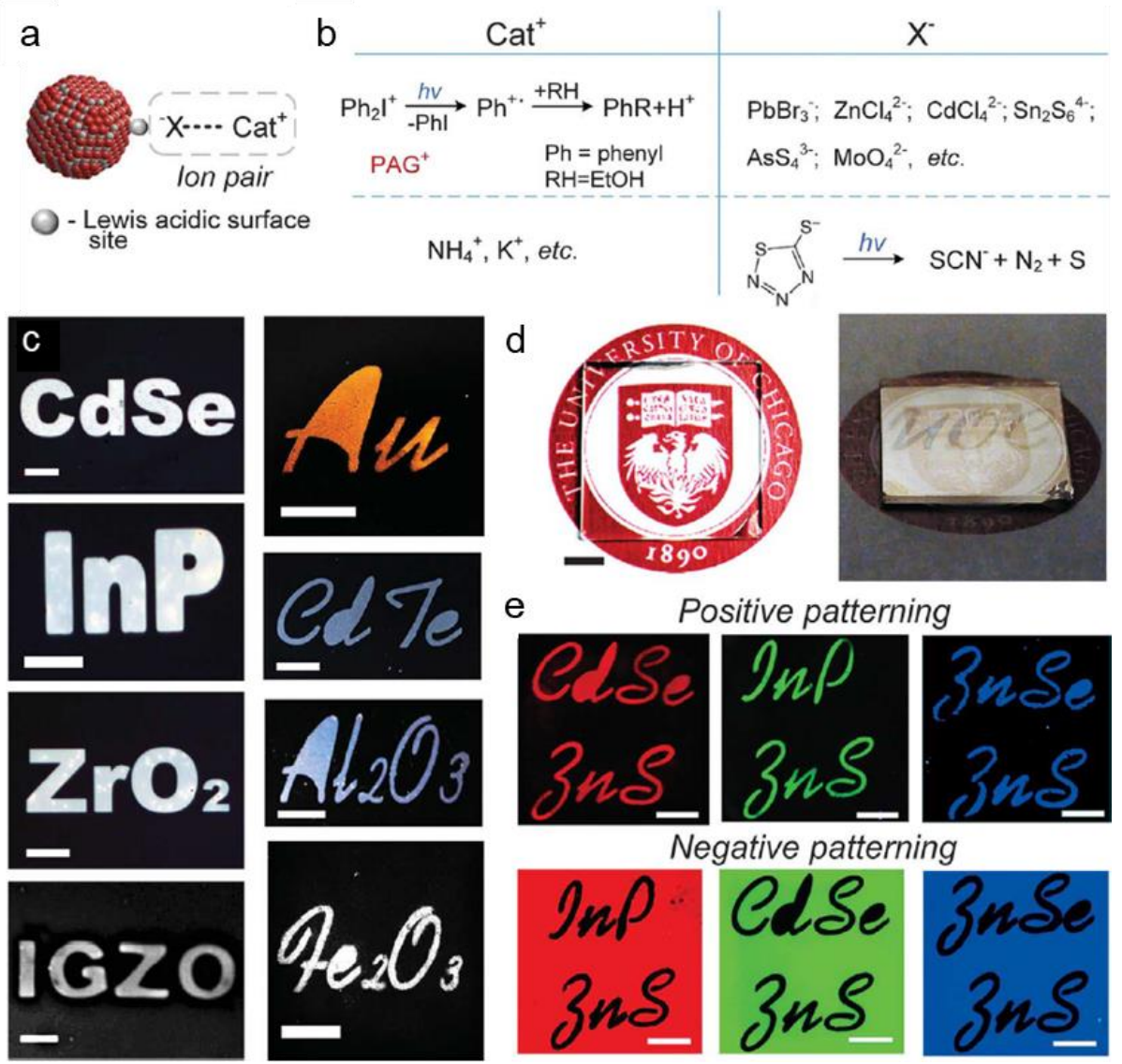

Figure 8. Direct optical lithography of functional inorganic nanomaterials with photosensitive inorganic ligands. ${ }^{23}$ a. Schematic illustration of QDs with ion pair surface ligands. b. Two complementary approaches to design of photosensitive inorganic ligands. c. Different materials patterned by using $\mathrm{PAG}^{+}$-based ligands $(\mathrm{CdTe}$, $\mathrm{ZrO}_{2}, \mathrm{Fe}_{2} \mathrm{O}_{3}$, and IGZO) and $\mathrm{CS}_{2} \mathrm{~N}_{3}{ }^{-}$ligands (CdSe, InP, $\mathrm{Au}$, and $\left.\mathrm{Al}_{2} \mathrm{O}_{3}\right)$. Scale bars, $100 \mathrm{~mm}$ (left column); $5 \mathrm{~mm}$ (right column). (d) $\mathrm{CeO}_{2}$ nanocrystals patterned on a glass substrate by using $\left(\mathrm{Ph}_{2} \mathrm{I}\right)_{2} \mathrm{MoO}_{4}$ ligands. Scale bar, $5 \mathrm{~mm}$. e. (Top) Positive patterns of $\mathrm{CdSe} / \mathrm{ZnS}$ (red), InP/ZnS (green), and $\mathrm{ZnSe} / \mathrm{ZnS}$ (blue) core-shell QDs patterned with $\mathrm{NH}_{4} \mathrm{CS}_{2} \mathrm{~N}_{3}$ ligands. (Bottom) Negative patterns of oleate-capped $\mathrm{InP} / \mathrm{ZnS}$ (red), $\mathrm{CdSe} / \mathrm{ZnS}$ (green), and $\mathrm{ZnSe} / \mathrm{ZnS}$ (blue) core-shell QDs patterned by using $\left(\mathrm{p}-\mathrm{CH}_{3} \mathrm{~S}-\mathrm{C}_{6} \mathrm{H}_{4}\right)\left(\mathrm{C}_{6} \mathrm{H}_{5}\right)_{2} \mathrm{~S}^{+} \mathrm{OTf}^{-}$(OTf, triflate) PAG. Scale bars, $5 \mathrm{~mm}$. 


\subsection{Nanoparticle self-assembly at fluid interfaces}

The most fundamental approach to create a large scale of self-assembled QD superlattice is by drop-casting the colloidal nanocrystal suspension onto a target substrate or a liquid subphase and allowing the solvent to evaporate. The evaporation rate of the solvent can be tuned by adjusting the volatility of the solvent or by controlling the evaporation rate in a sub-saturated vapor environment. Several works have previously shown that the self-assembly, disordering and reassembly of superlattices can be controlled through dynamic adjustments of the solvent vapor saturation. ${ }^{24-26}$ Interface effects are known to play a key role in the nucleation and growth processes of atomic crystals and QD superlattices. The formation of QD superlattices from thin liquid films is generally dominated by heterogeneous nucleation at a two-phase interface, e.g., the interface between two immiscible liquids, ${ }^{27}$ liquid/solid (l/s), ${ }^{28}$ or liquid/gas (l/g). ${ }^{29}$ Earlier studies by Whitham et al. demonstrated self-assembly of colloidal nanocrystals results in distinct superlattice polymorphs formed at $1 / \mathrm{g}$ interface shown in Figure $9 .{ }^{9}$ One attractive advantage of QD assembly at a fluid interface is the ability to tune the interfacial energies and modify ligand chemistries of the colloidal particles adsorbed to the fluid interface. 


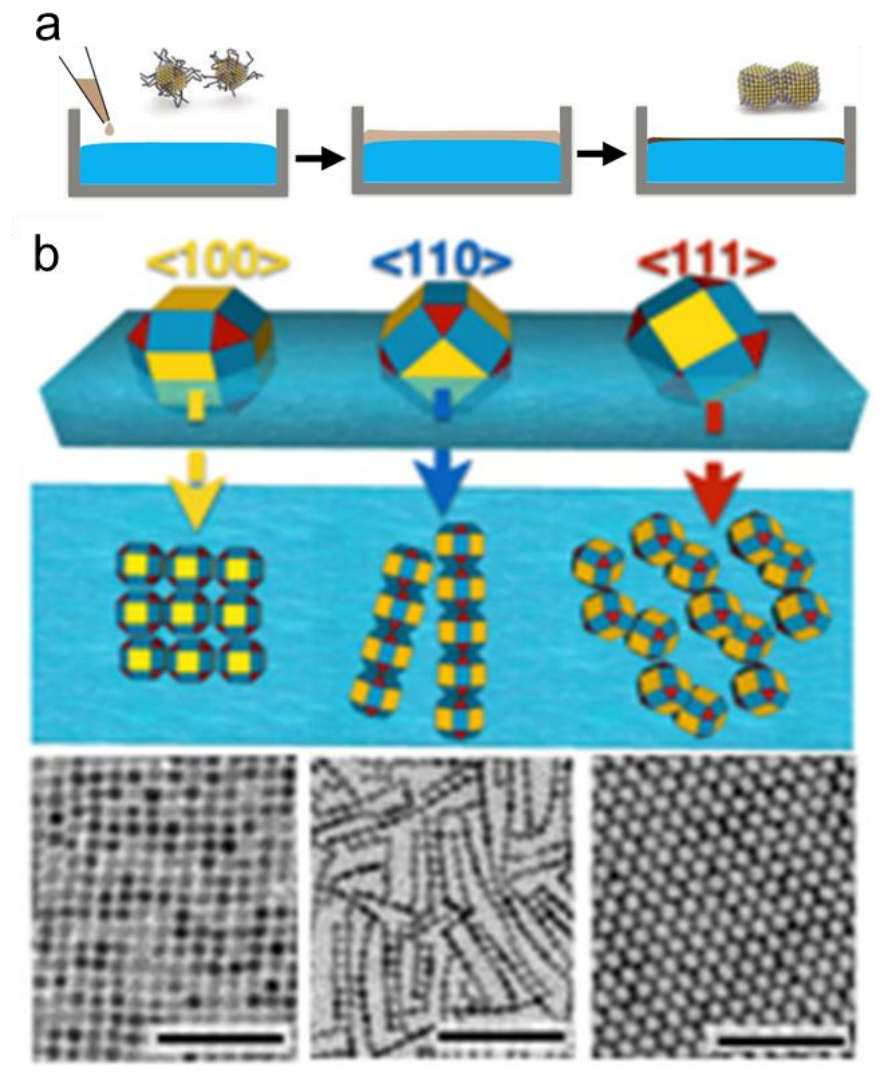

Figure 9. a. QD orientation (top) and multi-particle interactions at the fluid interface (middle) dictates the QD superstructure. TEM of square, line, and hexagonal structures. b. Directed assembly and attachment of QD at a liquid-liquid interface.

Scale bars: 50nm (bottom) ${ }^{9}$

\subsection{Optically-driven reorganization of colloidal assemblies}

In this work, we exploit the advantageous aspect of forming a Langmuir layer of colloidal QDs at a liquid interface and novel photolithographic patterning concepts to drive the superlattice structure transformation of QDs and designed pattern formation at the same time. We demonstrate that photogenerated acids 'deprotect' the colloidal QDs by reducing their ligand coverage, which in turn effects the interaction potential 
between proximate QDs and drives the transformation from a hexagonal to a square superlattice symmetry. We describe the processing method introduced in this work as optically-driven reorganization of colloidal assemblies (ORCA). Beyond the opportunity to gain an in-depth understanding of the fundamental relationship between QD ligand coverage and superlattice structure, we see the ORCA method and a promising protocol to create photo-programmable superlattice structure with significant technological potential by virtue of the underlying relationship between the superlattice structure and their emerging optoelectronic properties. 


\section{Chapter 2 \\ Experimental method}

In this chapter we report on the experimental methods used during this thesis both in the sample patterning and characterization. An important part will be focused on the experimental setup of ORCA process at liquid interface and best operation conditions for ORCA process such as concentration and volume. Spectroscopy (UV-vis and FTIR), microscopy (SEM and TEM) techniques used during the work and conductivity measurements are included in this chapter. Most of the instruments described are located in the School of Chemical and Biomolecular Engineering and at Cornell Center for Materials Research (CCMR).

\subsection{Material synthesis}

\subsubsection{Chemicals}

Oleic acid (OA, 90\%, Aldrich), 1-octadecene (ODE, 90\%, Aldrich), bis(trimethylsilylmethyl) sulfide ((TMS) ${ }_{2} \mathrm{~S},>98 \%$, Aldrich), trioctylphosphine (TOP, 98\%, Strem), lead oxide (PbO, Acros organics, > 99.9\%), 8-Hydroxypyrene-1,3,6trisulfonic acid trisodium salt (Pyranine, $\geq 97 \%$, Sigma), Diphenyliodonium chloride 
$\left(\mathrm{Ph}_{2} \mathrm{I}-\mathrm{Cl}, \geq 98.0 \%\right.$, Aldrich), Ethylene glycol (EG, 99.8\%, Sigma), methanol ( $\geq 99.8 \%$, VWR), toluene (99.9\%, Sigma), hexane (Sigma, 95\%).

\subsubsection{Synthesis of PbS QDs}

OA capped PbS QDs were synthesized following a procedure with high-accuracy size control. ${ }^{30}$ First, in a $100 \mathrm{ml}$ three-neck flask, $0.45 \mathrm{~g} \mathrm{PbO}, 20 \mathrm{~g} \mathrm{OA}$, and $10 \mathrm{~g}$ ODE were loaded and heated to $105^{\circ} \mathrm{C}$ for 20 min under vacuum to obtain a clear solution. Then the temperature was adjusted to $120{ }^{\circ} \mathrm{C}$. When reached the desired temperature, $210 \mu \mathrm{L}$ of (TMS) 2 S diluted in $5 \mathrm{~mL}$ of ODE was quickly injected into the flask (within 30 seconds). Immediately before injection, the heating mantle was removed. And the flask was cooled down by cold water 1.5 mins after injection to quench the reaction. Purifying PbS QDs is done through centrifugation with hexane (or toluene) as the solvent and ethanol (or methanol) as the counter solvent. Finally, PbS QDs was redissolved in toluene and stored in glovebox.

\subsubsection{Synthesis of CdSe QDs}

OA- capped CdSe QDs were synthesized by a hot-injection method. ${ }^{31}$ First, in a 100 $\mathrm{ml}$ three-neck flask, $64 \mathrm{ml}$ octadecene (ODE), $5.54 \mathrm{~g}$ OA and $0.51 \mathrm{~g} \mathrm{CdO}$ was loaded and heated gradually to $180{ }^{\circ} \mathrm{C}$ to form a clear solution under $\mathrm{N}_{2}$ atmosphere. At same time, TOP-Se solution was prepare in the glovebox by dissolving $1.3 \mathrm{mmol}$ Se powder and $0.5 \mathrm{~g}$ trioctylphosphine in $10 \mathrm{ml} \mathrm{ODE}$ and stirring more than $1 \mathrm{~h}$. When reached the desired temperature, TOP-Se solution was quickly injected into the flask. Then, the heater was removed $1.5 \mathrm{~min}$ later and cooled the system by immersed the flask in cold water to stop the reaction. Purifying CdSe QDs was done through centrifugation with hexane (or toluene) as the solvent and ethanol (or methanol) as the counter solvent. 
Finally, the particles were dissolved in toluene for future use.

\subsubsection{Synthesis of photoacid generator (PAG)}

$\mathrm{Ph}_{2} \mathrm{I}$-pyranine was synthesized following Tarumoto's method ${ }^{32} 262 \mathrm{mg}(0.5 \mathrm{mmol})$ Pyranine and $474.8 \mathrm{mg}(1.5 \mathrm{mmol}) \mathrm{Ph}_{2} \mathrm{I}-\mathrm{Cl}$ were mixed in DI water and stirred at $40{ }^{\circ} \mathrm{C}$ for $2 \mathrm{~h}$. The mixture would become cloudy. The precipitate was separated by centrifugation and washed three times with DI water. The purified product was dissolved into ethanol and stocked in the dark. The UV-vis absorption spectrum of $\mathrm{Ph}_{2} \mathrm{I}$-pyranine is shown in Figure 10.

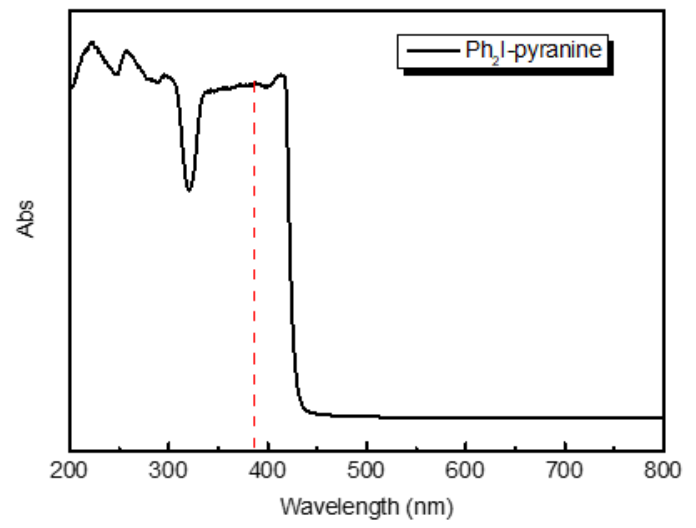

Figure 10. UV-vis absorption spectrum of $\mathrm{Ph}_{2} \mathrm{I}$-pyranine.

\subsection{Verification experiment of the chemistry}

Before designing and installing the experimental setup of ORCA process, we first spin coated materials on a Si wafer and expose the wafer to $385 \mathrm{~nm}$ UV light with designed pattern (A star, Letter A, Chinese character '远' and Cornell university logo). The materials used for spin coating are list in Table 1. As shown in Figure 11, we can clearly see the designed pattern on the wafer after 30 seconds of UV exposure which 
verified that this chemistry does work with $\mathrm{PbS}$ QDs (with oleic acid ligands) and $\mathrm{Ph}_{2} \mathrm{I}$-pyranine. Thus, we can continue our research work after that.

Table 1. Spin coating conditions.

\begin{tabular}{cccc}
\hline $\mathrm{V}_{\text {Methanol }}(\mu \mathrm{L})$ & $\mathrm{V}_{\text {toluene }}(\mu \mathrm{L})$ & $\mathrm{C}_{\mathrm{PbS}}(\mathrm{g} / \mathrm{L})$ & $\mathrm{C}_{\text {Ph2I-pyranine }}(\mathrm{g} / \mathrm{L})$ \\
\hline 65 & 65 & 2.7 & 4 \\
\hline
\end{tabular}

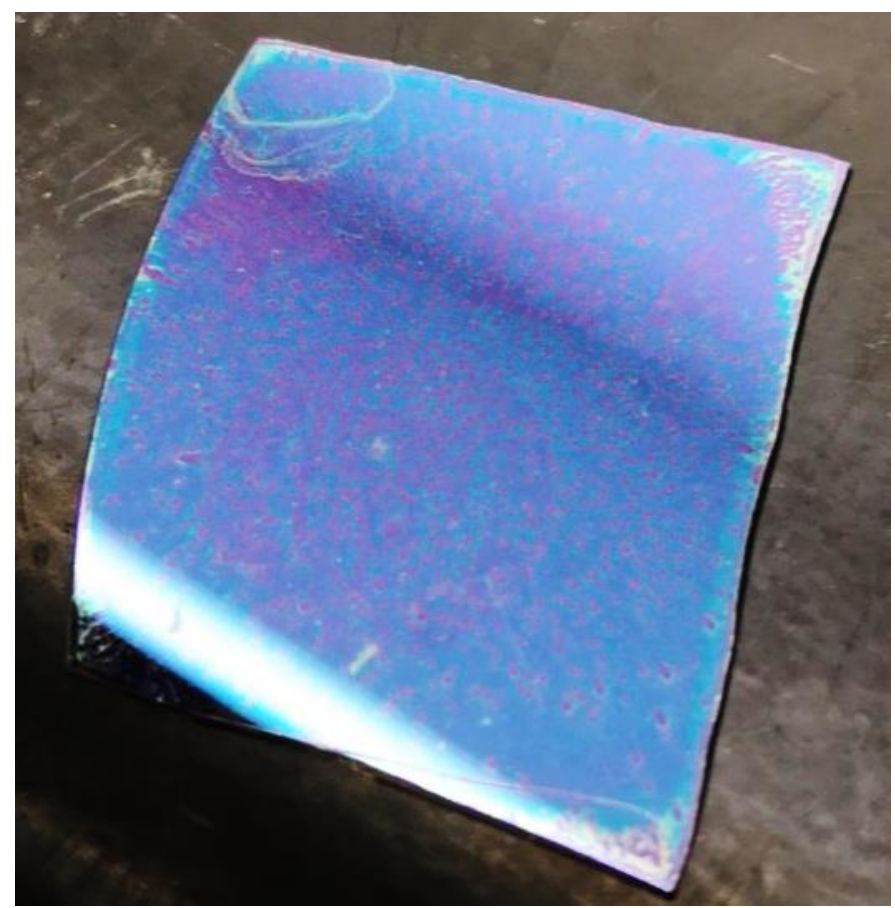

Figure 11. Spin coating results on silicon wafer. (A star, Letter A, Chinese character '远' and Cornell university logo were on the wafer)

\subsection{Design of experimental setup and experimental material conditions}

Interfaces of immiscible liquids as ideal means for the self-assembly of nanoparticles. ${ }^{33}$ It offers adequate stabilization of the nanoparticles with a high degree of organizational selectivity. Besides ultra-thin structures such as monolayer with large 
lateral sizes can also be generated at liquid-liquid interface. ${ }^{34}$ Therefore, we conducted the ORCA process at a liquid interface formed by hexane and ethylene glycol (EG). The sketch of setup and overall procedure are shown in Figure 12.

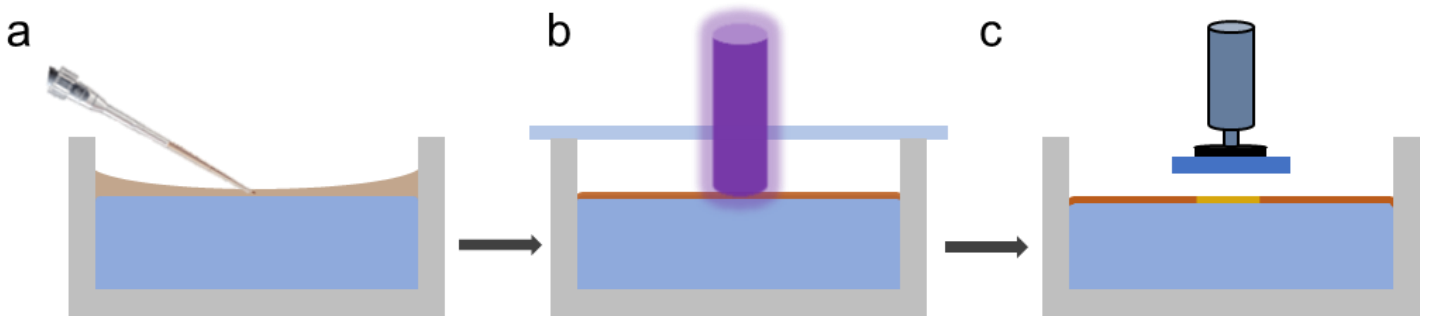

Figure 12. Sketch and procedure for ORCA process. a. Adding subphase $\left(\mathrm{Ph}_{2} \mathrm{I}-\right.$ pyranine in EG) and top phase (PbS capped with OA ligands in hexane). b. UV exposure after evaporation of hexane with covered quartz. c. Transferring.

As displayed, photoacid generator (PAG), $\mathrm{Ph}_{2} \mathrm{I}-$ pyranine solution was added to $1.5 \mathrm{~cm}$ $* 1.5 \mathrm{~cm} * 1.5 \mathrm{~cm}$ Teflon well. Then oleic acid (OA) capped PbS QDs in hexane was spread onto EG and quickly covered by quartz. $\mathrm{Ph}_{2} \mathrm{I}$-pyranine has a wide absorption peak from around $440 \mathrm{~nm}$ to $200 \mathrm{~nm}$ as displayed in Figure 10. Thus, we employed a $385 \mathrm{~nm}$ digital light projector (DLP) to emit a certain pattern of UV light top down. The DLP we used is shown in Figure 13. After tens of seconds of light exposure, the pattern is formed on top of the EG, visible to eye due to a darker color than the surrounding area. The films are transferred to a substrate using stamping (LangmuirSchaeffer method). 


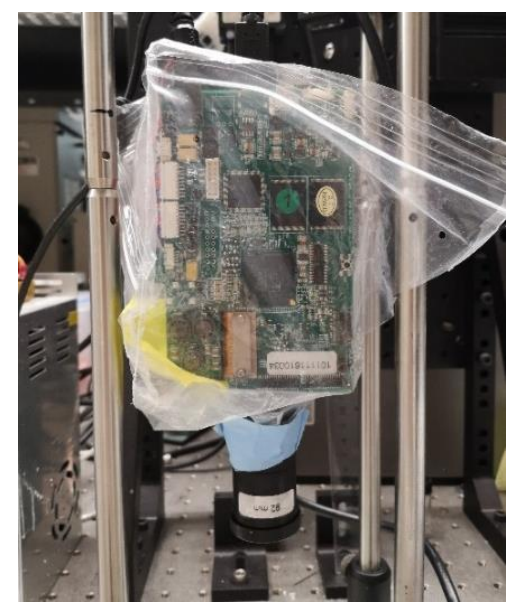

Figure 13. $385 \mathrm{~nm}$ digital light projector (DLP)

\subsection{Modify operation conditions for ORCA process}

\subsubsection{Concentration range of photoacid generator (PAG)}

To get a clear pattern at the liquid interface, we first exposed the system under UV light with fixed concentrate of $\mathrm{PbS} \mathrm{QDs}$ solution and exposure time. By varing the concentration of PAG and comparing the printed pattern, we could find the best concentration of PAG.

Table 2. Experimental results for different $\mathrm{Ph}_{2} \mathrm{I}$-pyranine concentration. (Adding $1 \mathrm{ml}$ subphase $20 \mu 11.01 \mathrm{~g} / \mathrm{PbS}$ (in toluene), observing results after 2 mins UV exposure).

\begin{tabular}{ccc}
\hline Set & Results & C 2 Ph2I-pyranine $(\mathrm{g} / \mathrm{l})$ \\
\hline 1 & None & 0.17 \\
2 & None & 0.34 \\
3 & Hollow heart & 0.67 \\
4 & Not clear & 1.34 \\
5 & Yellow chemicals & 2.07 \\
\hline
\end{tabular}


In this case, the volume of subphase was $1 \mathrm{~mL}$ and $20 \mu 11.01 \mathrm{~g} / \mathrm{L} \mathrm{PbS}$ (in toluene) was added. Then observe the experimental results after 2 mins UV exposure.

We could see patterns formed when concentration of $\mathrm{Ph}_{2} \mathrm{I}$-pyranine was $0.67 \mathrm{~g} / \mathrm{l}$, but no clear patterns when the concentration was larger or smaller than $0.67 \mathrm{~g} / \mathrm{l}$. Thus, it was concluded that the best concentration of $\mathrm{Ph}_{2} \mathrm{I}$-pyranine was at somewhere around $0.67 \mathrm{~g} / \mathrm{l}$.

\subsubsection{Concentration range of $\mathrm{PbS}$}

To find the best concentration range of $\mathrm{PbS}$ QDs solution, we fitst calculated the number of $\mathrm{PbS}$ QDs particles for a monolayer at the liquid interface in Teflon well. For $8.3 \mathrm{~nm}$ PbS QDs, $2.15 \times 10^{12} \mathrm{PbS}$ QDs will be needed to form a monolayer on EG surface ideally. The evaporation of colloidal solvents would result in superlattice crystallization, thus the evaporation rate of the solvent dissolving PbS QDs would effect the process. ${ }^{35}$ We used hexane to dissolve PbS QDs because of its evaporation rate. The QD concentration was determined with optical absorption spectroscopy using the procedure of Moreels et al. ${ }^{36}$ We controled the volume of subphase within $5 \sim 10 \mu$ l, the evaporation time was within 10 minutes. As calculated, to form a 3-layer PbS QDs on EG, we need $10 \mu \mathrm{l}$ of $1.9 \mathrm{~g} / \mathrm{l} \mathrm{PbS}$ solution. We introdued $6.5 \mu \mathrm{L} 3 \mathrm{~g} / \mathrm{l} \mathrm{PbS}$ QDs in hexane on EG, and after hexane evaporating, we took a sample for Transmission electron microscopy (TEM). From Figure 14, we can see that PbS form a homogenious monolayer. 


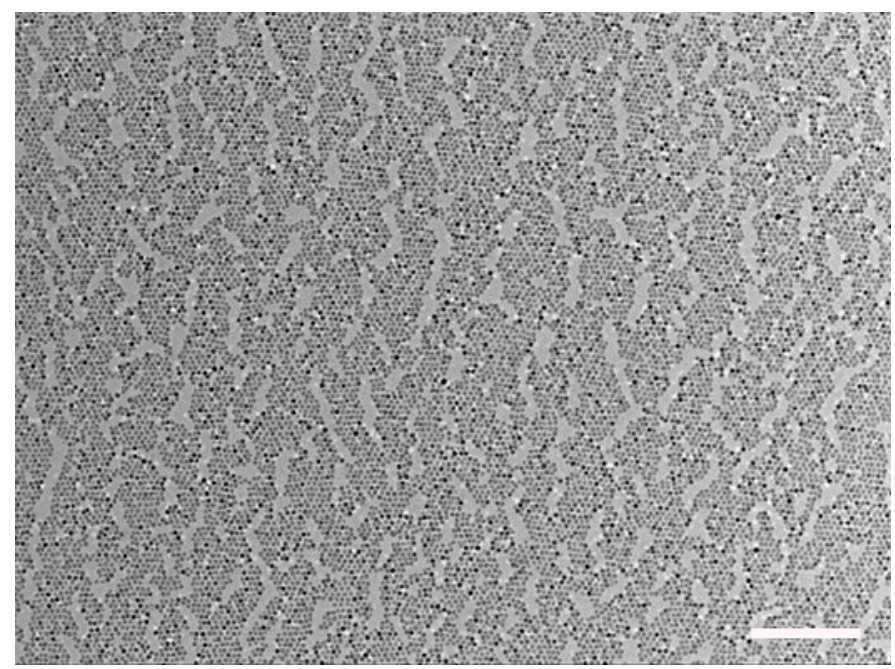

Figure 14. TEM image of $6.5 \mu \mathrm{L} 3 \mathrm{~g} / \mathrm{L}$ PbS QDs in hexane evaperting on EG. Scale bar: $200 \mathrm{~nm}$.

\subsubsection{Limiting thickness of PbS QDs}

To find the limited printing thickness of ORCA, we fixed the concentration of $\mathrm{PbS}$ QDs and varied the volume introdued on subphase. Meanwhile, other parameters such as concentation of PAG and exposure time remained the same. All samples were washed by methanol after transferring to remove EG. Figure 15 shows patterns on wafer of different cases and Table 3 shows the printed conditions for each case.

Table 3. Printed conditions for each case.

\begin{tabular}{ccccccc}
\hline & $\mathrm{V}_{\mathrm{PbS}}(\mathrm{ul})$ & $\mathrm{C}_{\mathrm{PbS}}(\mathrm{g} / \mathrm{l})$ & $\begin{array}{c}\text { Expected } \\
\text { layers }\end{array}$ & $\mathrm{V}_{\text {subphase }}(\mathrm{uL})$ & $\begin{array}{c}\mathrm{C}_{\text {Ph2I- }} \\
\text { pyranine }(\mathrm{g} / \mathrm{l})\end{array}$ & $\begin{array}{c}\text { Exposure } \\
\text { time }(\mathrm{s})\end{array}$ \\
\hline $\mathrm{a}$ & 10 & & $1 \sim 2$ & & & \\
$\mathrm{~b}$ & 20 & $3 \mathrm{~h}$ & $\sim 3$ & & & \\
$\mathrm{c}$ & 30 & & $4 \sim 5$ & 1000 & 0.50 & 120 \\
$\mathrm{~d}$ & 40 & & $\sim 6$ & & & \\
\hline
\end{tabular}



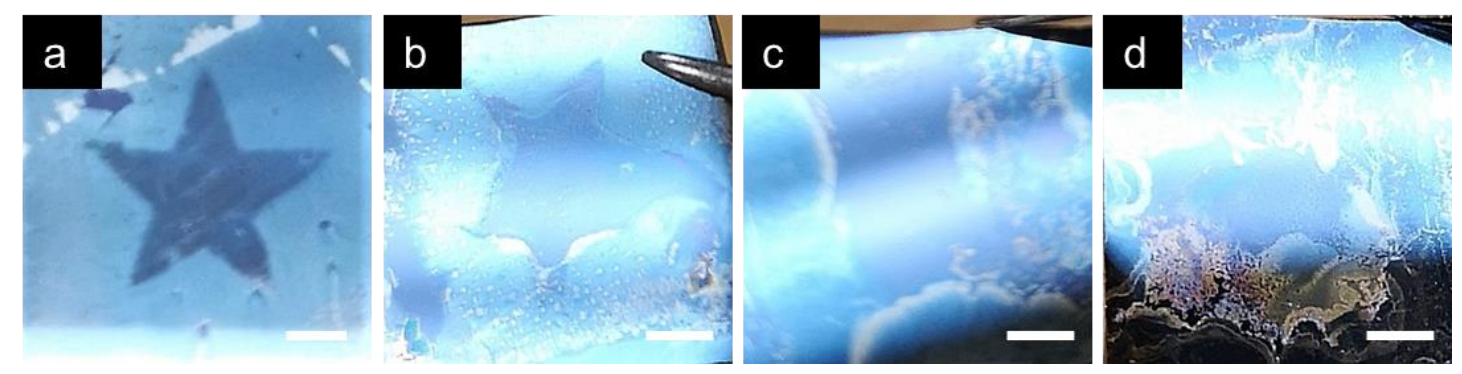

Figure 15. Printed pattern (star) on wafer. a. 1 2 layers of $\mathrm{PbS}$. b. $\sim 3$ layers of $\mathrm{PbS}$. c. 4 5 layers of PbS. d. 6 layers of PbS. Scale bars: $1 \mathrm{~mm}$.

In case $a$, we can see a clear star on wafer. In case b, the star became out of shape. And as there were more $\mathrm{PbS}$ layers, it can not form a clear pattern on EG. The results are repeatable. Thus, in this research work, we target on $\mathrm{PbS}$ monolayer. We will mainly discuss the limited resolution of printed patterns in Charpter 3.

\subsubsection{Transferring printed patterns}

How to transfer printed patterns reliably was the main concern in the ORCA process. We used Langmuir-Schaeffer method to stamp printed patterns to substrates. There are two approaches we think could improve the transferring process. First, it was assumed that nanoparticles would have more mobility as they are in the solution and make the transferring more unstable. Thus, we compared transferring patterns in dry and wet conditions. Dry condition refers to exposing to UV and transferring patterns after evaporation of hexane, while wet condition means exposing and transferring with hexane remaining. The results are displayed in Figure 16. For a, b and d, e, we added $15 \mu \mathrm{l} 3 \mathrm{~g} / \mathrm{l} \mathrm{PbS}$ QDs on $1 \mathrm{ml} 0.8 \mathrm{~g} / \mathrm{l} \mathrm{Ph} 2 \mathrm{I}-$ pyranine and exposed to UV for 70 seconds. $\mathrm{b}$ and e are figures of liquid interface after pattern transferring, $\mathrm{c}$ and $\mathrm{h}$ show patterns on undoped silica wafer. In e, we observed that after attaching silica wafer to the liquid 
interface, the pattern was still at the interface and became out of shape. Besides, there was no pattern on wafer (Figure 16h). Thus, we confirmed that, to transfer patterns reliably, exposing and transferring after hexane is evaporated is more reasonable.
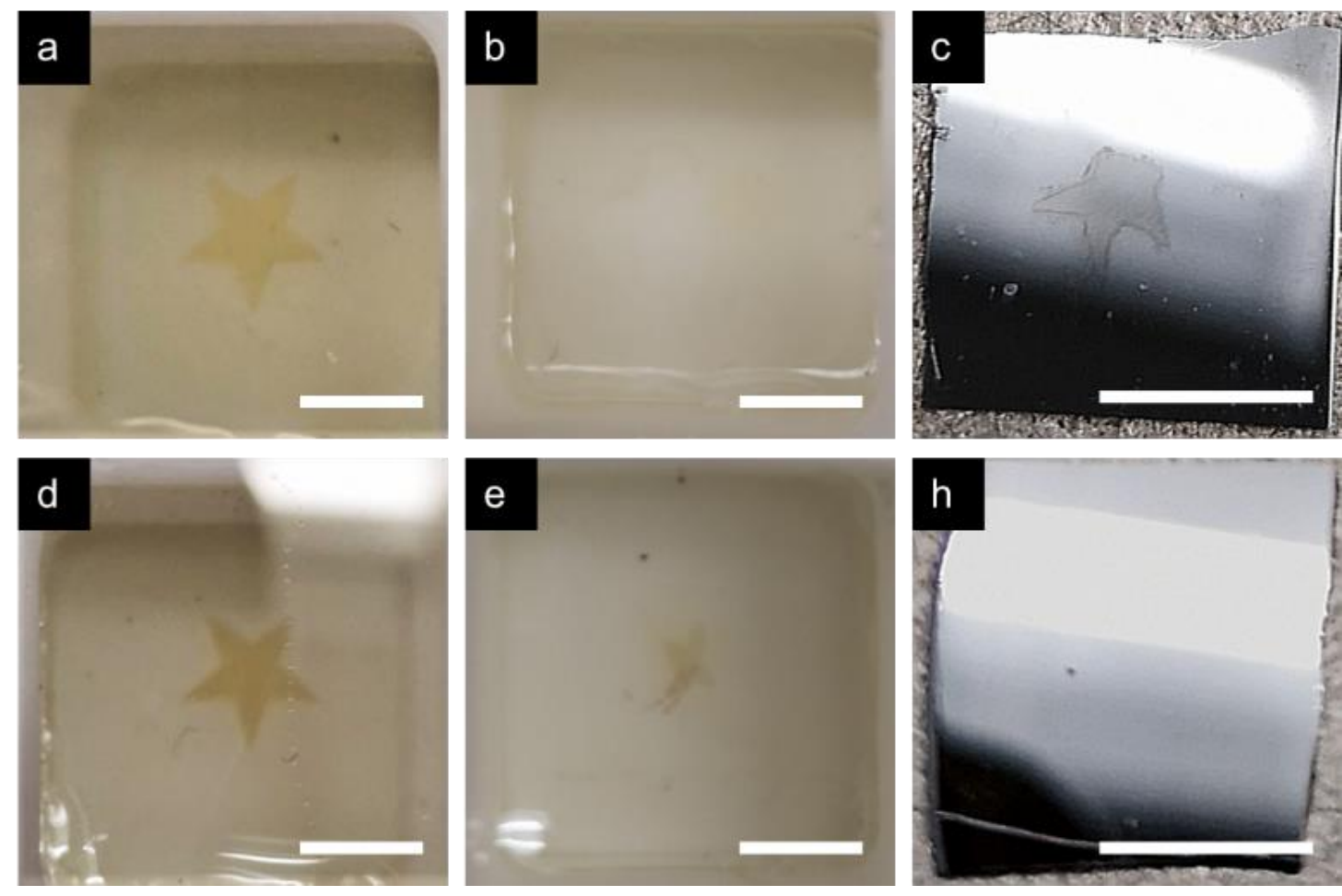

Figure 16. Comparison results of dry and wet cases. (a, d) Patterns after exposure, (b,

e) After transferring to silicon wafer, (c, h) Patterns on silicon wafer. Scale bars: $5 \mathrm{~mm}$.

Second, we compared transferring reliability of different kinds of substrates, HMDS functionalized wafer (a), MPTMS functionalized wafer (b), undoped silica wafer (c), wet thermal oxide wafer (d) and glass. (3-mercaptopropyl) trimethoxysilane (MPTMS) and hexamethyldisilazane (HMDS) are often used to construct an adhesive layer on silica surface which helps to form nanocrystals monolayer. The results are presented in Figure 17. Compared a, b and c, HMDS and MPTMS do improve the adhesive ability but the transferred patterns were still unsatisfying. However, wet thermal oxide wafer 
shows good ability to transfer patterns reliably.
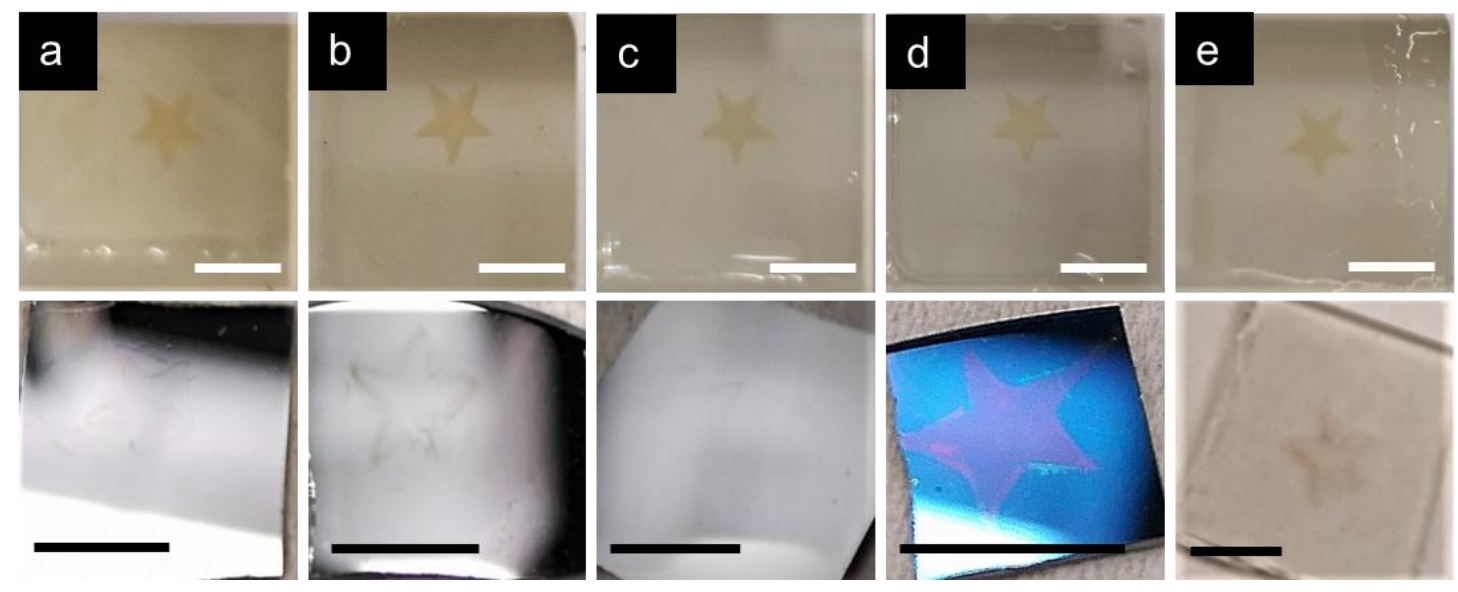

Figure 17. Comparison six kinds of substrates. a. HMDS functionalized wafer. b.

MPTMS functionalized wafer. c. undoped silica wafer. d. wet thermal oxide wafer. e. glass. Scale bars are $5 \mathrm{~mm}$.

\subsubsection{ORCA process}

In a typical experiment, firstly, the Teflon trough $(15 \mathrm{~mm} \times 15 \mathrm{~mm} \times 15 \mathrm{~mm})$ was filled with $1 \mathrm{ml}$ of a $0.5 \mathrm{~g} / \mathrm{L}$ PAG solution. Then PbS QDs dissolved in hexane were added to the fluid surface via a micropipette. In a typical experiment $6.5 \mu 1$ of a 3 $\mathrm{mg} / \mathrm{ml}$ solution was added. The amount of QDs was calculated to yield a single $\mathrm{PbS}$ monolayer. As the hexane evaporating, a flat film of PbS QDs can be formed on top of the EG, wherein the thickness is determined by the diffusion region and the volume introduced. ${ }^{37}$ The quality of the flat film would be influenced by factors such as surface tension and contact angle. Immediately after addition of the QD solution, the top of the trough was covered with a quartz slide to slow down the solvent evaporation rate and ensure formation of a uniform QD layer. After hexane were evaporated, we exposed it to UV light and certain pattern can formed. 


\subsection{Characterization techniques and sample preparation}

\subsubsection{Fourier transform infrared}

Fourier transform infrared (FTIR) spectra were acquired in transmission mode using a Bruker Vertex V80V Vacuum FTIR system. Four samples (0s, 15s, 30s, 120s) for FTIR characterization were prepared on quartz substrates. For the original sample (0s), we introduced $6.5 \mu \mathrm{L} 3 \mathrm{~g} / \mathrm{L} \mathrm{PbS}$ QDs solution on $0.5 \mathrm{~g} / \mathrm{L}$ PAG solution. Transferred QD film to quartz after hexane evaporation. Repeated five times to attach five layers on the same quartz. For the sample after ORCA process (15s, 30s, 120s), same volume and concentration of subphase and top phase were introduced. After hexane evaporation, QD films on top of PAG solution were exposed to UV light with corresponding time. Also repeating five times to make multilayer samples. Remaining EG on the quartz was rinsed by toluene. The results would be discussed in Chapter 3. Here, we attached measured FTIR spectra of Pyranine and OA as a reference (Figure 18).

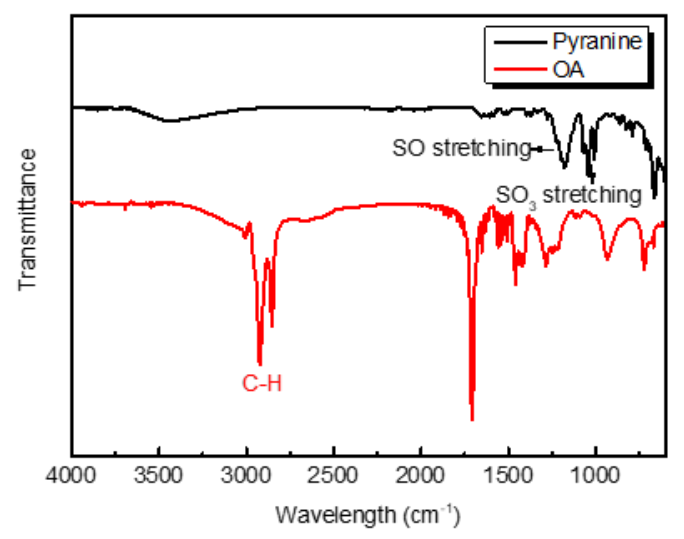

Figure 18. FTIR spectra for Pyranine and oleic acid.

\subsubsection{Optical absorption measurements}


Near-infrared (NIR) absorption spectra were acquired using a Cary 5000 UV-Vis-NIR spectrophotometer at room temperature. The spectra were baseline corrected.

Samples for optical characterization were prepared on glass substrates. In these four cases, the volume and concentration of subphase and top phase remained the same, namely $1 \mathrm{ml} 0.5 \mathrm{~g} / \mathrm{L} \mathrm{Ph}_{2} \mathrm{I}$-pyranine in $\mathrm{EG}$ and $10 \mu \mathrm{L} 2 \mathrm{~g} / \mathrm{L} \mathrm{PbS}$ in hexane. Other procedures of preparation were same as those for FTIR samples. The results would be discussed in Chapter 3.

\subsubsection{Transmission electron microscopy}

Transmission electron microscopy (TEM) images were obtained using a FEI T12 Spirit TEM STEM.

The results are displayed in Chapter 3. For Figure 24a, the subphase was $1 \mathrm{~g} / \mathrm{L} \mathrm{Ph}_{2} \mathrm{I}-$ pyranine solution and top phase was $4 \mu \mathrm{L} 3.82 \mathrm{~g} / \mathrm{L}$ PbS solution. Without UV exposure, but PbS QDs seated on subphase for 120 s after drying. Figure 24c was from same batch with Figure 25d. For Figure 25b-d, we fixed the concentration of $\mathrm{Ph}_{2} \mathrm{I}-$ pyranine and $\mathrm{PbS}$ at $0.5 \mathrm{~g} / \mathrm{L}$ and $3 \mathrm{~g} / \mathrm{L}$ and varied the photo-dosage by changing the exposure time. We added 6.5 $\mu \mathrm{L} \mathrm{PbS}$ ink to ensure the formation of $\mathrm{PbS}$ monolayer at liquid-liquid interface. Directly after different time of UV exposure (15s, 30s, 120s), we attached the carbon film coated, 200 Mesh, $\mathrm{Cu}$ TEM grids to the exposed EG surface. For the sample with 0s UV exposure (Figure 25a), we just attached the TEM grid to the liquid interface outside the printed star after $30 \mathrm{~s}$ UV exposure. Excess EG was removed by immersing the TEM grid into methanol and then dried each grid in the air. In Figure 26a-d, we quantified the 4-fold symmetry with different concentration of $\mathrm{Ph}_{2} \mathrm{I}$-pyranine. We fixed the exposure time at $30 \mathrm{~s}$ and varied the 
concentration from $0.25 \mathrm{~g} / \mathrm{L}$ to $2 \mathrm{~g} / \mathrm{L}$. The transferring and washing procedures were same as Figure 26a-d. And the samples were kept in glovebox before measuring.

\subsubsection{Two-terminal conductivity measurement}

To measure the difference of charge transport of $\mathrm{PbS}$ thin film before and after ORCA process, we constructed devices with different exposure time. The scheme of the devices is demonstrated in Figure 19.

\begin{tabular}{|c|c|c|}
\hline Conductor & QD (channel) & Conductor \\
\hline \multicolumn{3}{|c|}{ Substrate } \\
\hline
\end{tabular}

Figure 19. A schematic cross-sectional view of devices for measuring two-terminal conductivity.

For samples with ORCA process $(0 \mathrm{~s}, 15 \mathrm{~s}, 30 \mathrm{~s}, 60 \mathrm{~s})$, we transferred 5 layers of $\mathrm{PbS}$ thin film onto $\mathrm{Si}^{++} / \mathrm{SiO}_{2}(200 \mathrm{~nm})$ substrates carrying the electrode pattern $(50 \mathrm{~nm} \mathrm{Au})$. Lithographically defined interdigitated electrodes forming channels $5 \mu \mathrm{m}$ long and 1 $\mathrm{cm}$ wide were used. And the total area of each device including the electrodes is 6 $\mathrm{mm}^{2}$. Each PbS film was prepared in the same manner as that for FTIR and had same seating time on subphase. And after transferring, toluene was used to remove EG on 15s, 30s and 60s exposure devices. For comparison, we dropped cast original OA capped $\mathrm{PbS}$ solution onto another device. Then we tested the electronic conductivity for those devices by scanning drain voltage at fixed gate voltages $(0 \mathrm{~V})$ in the air. The output characteristics were reported in Figure 30 and would be discussed in Section 3.5 . 


\section{Chapter 3 \\ Results and discussion}

This chapter reports the results and discussions of optically-driven reorganization of colloidal assemblies (ORCA). The discussions are divided into four parts: mechanism of chemistry, macroscale study, microscale study and study of properties. The macroscale study focuses on resolution of printed patterns. The microscale study focuses on local assembly structures and the influence of light dosage on structure transformation. We show that the film structure can be tuned between six-fold and four-fold symmetry by controlling the exposure time. We study the optical and electrical properties of printed thin films and show the influence of photodosage on optical properties and electronic conductivity in details.

\subsection{Reaction mechanism of ORCA process}

The proposed mechanistic sequence of PAG photolysis and QD ligand removal is schematically illustrated in Figure 20. PAGs form acids via photodissociation upon illumination which is widely exploited in photolithography and recently reported to

direct pattern inorganic materials. ${ }^{23}$ Within the photoexposed regions, $\mathrm{Ph}_{2} \mathrm{I}$-pyranine 
decomposes to a pyranine group and three diphenyliodinium moieties which generate acid upon photolysis. The mechanism of acid generation is intramoleculor electron transfer from the excited pyranine group to the diphenyliodinium moieties. ${ }^{32}$ The photogenerated acid shifts the dynamic equilibrium of bound and free oleate ligands, ${ }^{38}$, 39 which results in the oleate on the QD surface to be replaced with oleic acid and diffuse from the QD surface into the EG subphase. Considering the large excess of the monoanion of ethylene glycol at the fluid interface, we assume that the 2hydroxyethanolate bind to freshly vacated sites on the QD surface. Hassinen et al. have previously reported that short-chain alcohols can induce substitution of X-type carboxylate ligands. ${ }^{38}$ Besides, Wang et al. reported that pyranine group could bind to $\mathrm{PbS}$ QD surface, which forms an ion pair with metal sites. ${ }^{23}$ However, we can not confirm that from FTIR. (see Figure S1, the exposed PbS QD thin flims don't have characteristic signal of $\mathrm{SO}$ and $\mathrm{SO}_{3}$ stretching of pyranine groups) 


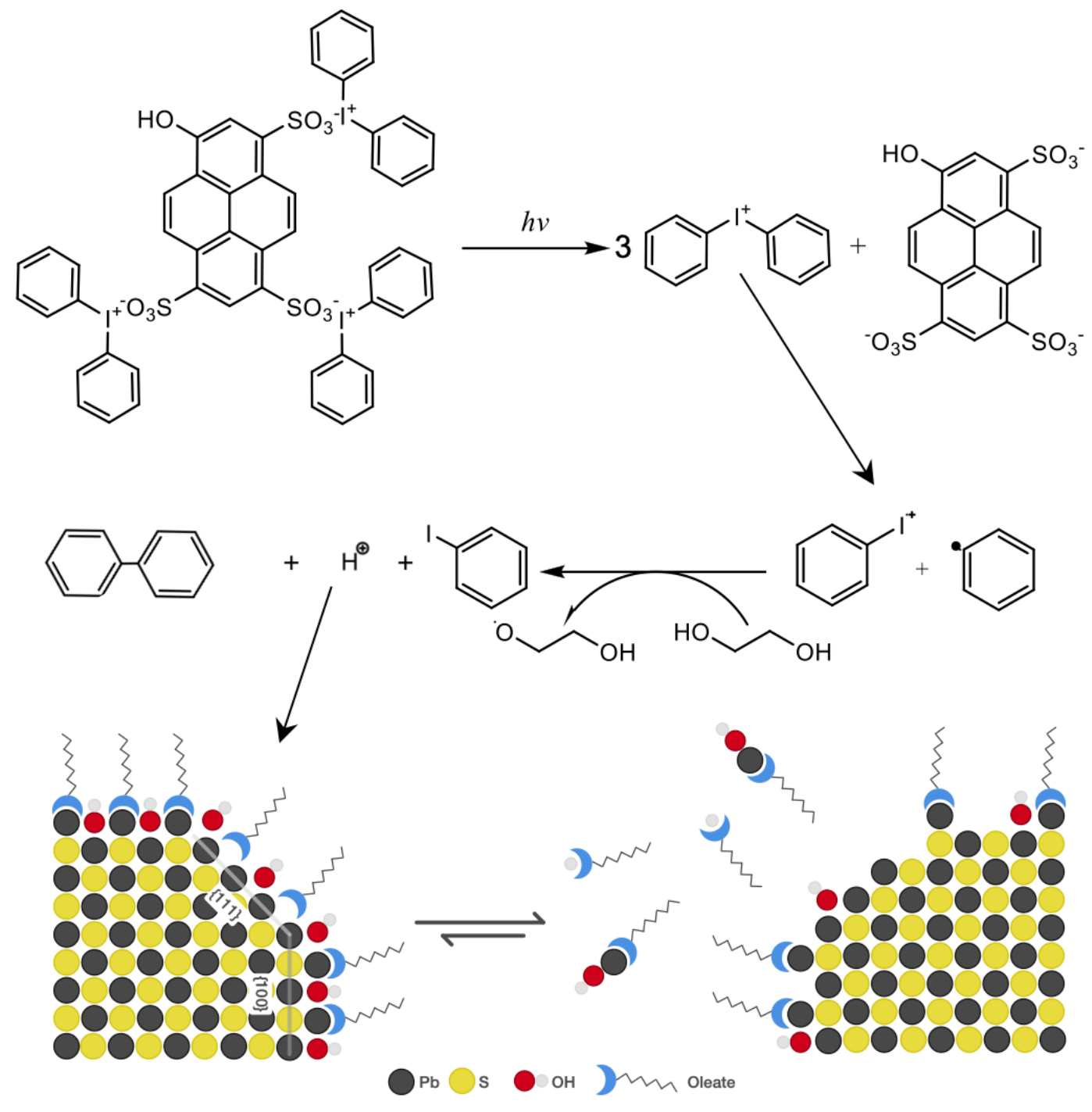

Figure 20. Mechanistic sequence of PAG photolysis and QD ligand removal of ORCA process.

\subsubsection{Analysis of generation photolysis and ligand removal rate}

As illustrated in Figure 21, the PAG initiated 'de-protection' of QD at the interface involves a series of sub-processes including:

(1) photogeneration of the acid,

(2) transport of the acid from volume of the subphase fluid to the QD at the interface, 
(3) acid catalyzed de-protection. (i.e., removal of surface-bound ligands), and

(4) transport of detached ligands (oleic acid and lead oleate) from the interface to the bulk EG subphase solution.

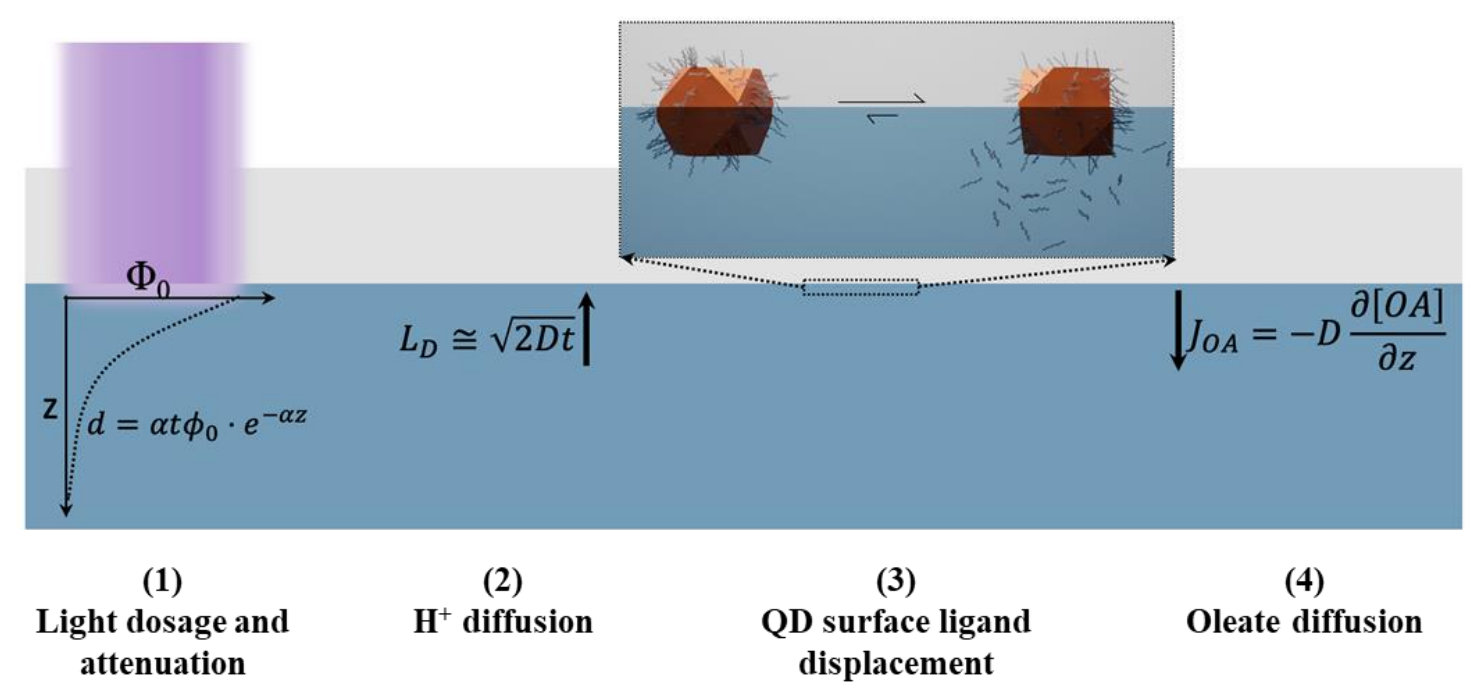

Figure 21. Sequence of coupled sub-processes involved in ORCA processing.

With the aid of the analytical model detailed below, we sought to understand which of these sub-processes limit the overall process.

We determined the extent of ligand removal in the photochemical reaction. FTIR spectroscopy has been previously used to estimate oleate coverage. ${ }^{40,41}$ Based on FTIR data discussed in Figure 23, we can approximate the ligand removal based on the reduction of $\mathrm{CH}$ stretching intensity.

Accordingly, the ligand coverage is reduced from 1000 / QD to 400/ QD during the $15 \mathrm{~s}\left(75 \mathrm{~mJ} / \mathrm{cm}^{2}\right)$ photodosage. Assuming a $\sim 8 \mathrm{~nm} \mathrm{PbS}$ QDs with oleate ligand coverage and a $\sim 11 \mathrm{~nm}$ center-to-center spacing, we have surface coverage of about $100 \mathrm{~nm}^{2} /$ dot. This means that during the $15 \mathrm{~s}$ exposure, $\sim 6 \times 10^{14} \mathrm{OA} / \mathrm{cm}^{2}$ are 
removed, corresponding to a flux of oleate ligands on the order of $4 \times 10^{13} \mathrm{OA} / \mathrm{cm}^{2} / \mathrm{s}$. Below, we examine which sub-process limits this overall flux.

The light used to initiate the ligand removal is attenuated by the PAG in the EG subphase. To understand how deep into the EG subphase PAG photolysis occurs, we modeled the light attenuation as follows:

$\Phi=$ photon flux, $\Phi_{0}=$ initial photon flux on EG surface, $5 \mathrm{~mW} \cdot \mathrm{cm}^{-1}$

$\varepsilon=$ molar attenuation coefficient, $2.24 \times 10^{4} \mathrm{M}^{-1} \cdot \mathrm{cm}^{-1}[3]$

$\mathrm{c}=$ concentration of PAG, $3.7 \times 10^{-4} \mathrm{M}$

$\alpha=$ absorptivity

$\mathrm{A}=$ absorbance

$\mathrm{d}=$ absorbed dosage

$\mathrm{z}$ presents the downward direction of the photon flux from the EG surface. The $\mathrm{QD} / \mathrm{EG}$ interface is defined as $\mathrm{z}=0$. The attenuation of photon flux along $z$ direction:

$$
\begin{aligned}
& \phi(z)=\phi_{0} \cdot e^{-\alpha z} \\
& A=\frac{\partial \phi}{\partial z}=\alpha \phi_{0} \cdot e^{-\alpha z} \\
& d=A t=\alpha t \phi_{0} \cdot e^{-\alpha z}
\end{aligned}
$$

critical dosage $\left(d_{c}\right)$ up to $z_{\mathcal{c}}$ should equal to the energy needed for PAG decomposing.

$$
\begin{gathered}
d_{c}=\alpha t \phi_{0} \cdot e^{-\alpha z_{c}}=310 / 0.63 \mathrm{~kJ} \cdot \mathrm{mol}^{-1}=492 \mathrm{~kJ} \cdot \mathrm{mol}^{-132} \\
\alpha=\varepsilon \cdot c=2.24 \times 10^{4} \mathrm{M}^{-1} \cdot \mathrm{cm}^{-1} \times 3.7 \times 10^{-4} \mathrm{M}=8.3 \mathrm{~cm}^{-1} \\
\phi_{0}=5 \mathrm{~mW} \cdot \mathrm{cm}^{-2}=5 \times 10^{-3} \mathrm{~J} \cdot \mathrm{s}^{-1} \cdot \mathrm{cm}^{-2} \\
\lambda=385 \mathrm{~nm} \rightarrow E=3.22 \mathrm{eV}=5.16 \times 10^{-19} \mathrm{~J} / \text { photon }
\end{gathered}
$$




$$
\begin{aligned}
& \phi_{0}=\left(5 \times 10^{-3} \mathrm{~J} \cdot \mathrm{s}^{-1} \cdot \mathrm{cm}^{-2}\right) \times\left(\frac{\text { photon }}{5.16 \times 10^{-19} \mathrm{~J}}\right) \\
= & 9.6 \times 10^{15} \text { photon } \cdot \mathrm{cm}^{-2} \cdot \mathrm{s}^{-1}
\end{aligned}
$$

We define the critical depth, $\mathrm{z}_{\mathrm{c}}$ as the depth to which the local light intensity is sufficient to degrade the PAG.

For a $15 \mathrm{~s}$ of UV exposure $t=15 \mathrm{~s}$,

$$
\begin{aligned}
& d_{c}=(\left.8.3 \mathrm{~cm}^{-1}\right) \times(15 \mathrm{~s}) \times\left(9.6 \times 10^{15} \text { photon } \cdot \mathrm{cm}^{-2} \cdot \mathrm{s}^{-1}\right) \times e^{-8.3 z_{c}} \\
&=\left(492 \mathrm{~kJ} \cdot \mathrm{mol}^{-1}\right) \times(c) \\
&=\left(8.3 \mathrm{~cm}^{-1}\right) \times(15 \mathrm{~s}) \times\left(5 \times 10^{-3} \mathrm{~J} \cdot \mathrm{s}^{-1} \cdot \mathrm{cm}^{-2}\right) \times e^{-8.3 z_{c}} \\
&=\left(\frac{492 \mathrm{~kJ}}{\mathrm{~mol}}\right) \times\left(\frac{3.7 \times 10^{-4} \mathrm{~mol}}{1000 \mathrm{~cm}^{3}}\right)=0.621 \mathrm{~J} \cdot \mathrm{cm}^{-3} \times e^{-8.3 z_{c}} \\
&=0.182 \mathrm{~J} \cdot \mathrm{cm}^{-3} \\
& z_{c}=0.15 \mathrm{~cm}=1.5 \mathrm{~mm}
\end{aligned}
$$

Therefore, up to a depth of $1.5 \mathrm{~mm}$ PAG dissociates under these conditions. Note that this model assumes a quantum yield (QY) of unity, but even with a QY of about 0.5 , the critical dosage depth would still be on the order of a millimeter.

The second sub-process illustrated in Figure 21 is the transport of photogenerated acid from the bulk EG subphase to the surface at which QDs are assembled. We modeled this transport as basic Fickian diffusion. In this case the mean diffusion length is given by:

$$
\begin{gathered}
L_{D} \cong \sqrt{2 D t} \\
t=15 s, D \approx 10^{-6} \mathrm{~cm}^{2} \cdot s^{-1} \\
L_{D} \simeq 55 \mu m^{42}
\end{gathered}
$$


This analysis shows that the diffusion length is much shorter than the critical light dosage depth $\left(\mathrm{L}_{\mathrm{D}}<<\mathrm{z}_{\mathrm{c}}\right)$. We can therefore conclude that PAG within the $55 \mu \mathrm{m}$ depth $\left(\mathrm{L}_{\mathrm{D}}\right)$ is degraded. Furthermore, we conclude that the process is not limited by light dosage (under these conditions). The amount of photogenerated acid that can diffuse to the QD interface during the process is given by:

$$
\begin{aligned}
& {\left[H^{+}\right]=\left((\text {area }) \cdot\left(L_{D}\right)\right)[P A G]=3 \times\left(1 \mathrm{~cm}^{2}\right) \times(55 \mu \mathrm{m}) \times\left(\frac{3.7 \times 10^{-4} \mathrm{~mol}}{1000 \mathrm{~cm}^{3}}\right)} \\
& {\left[\mathrm{H}^{+}\right]=3.6 \times 10^{15} \mathrm{~cm}^{-2}}
\end{aligned}
$$

Thus, on a per area basis the available acid concentration is $3.6 \times 10^{15} \mathrm{H}^{+} / \mathrm{cm}^{2}$, which exceeds the oleate removal $\left(6 \times 10^{14} \mathrm{OA} / \mathrm{cm}^{2}\right.$, vide supra $)$ by about a factor of 6 ; we can therefore conclude that the ORCA process is not limited by transport of $\mathrm{H}^{+}$to the interface.

The last step in the model illustrated in Figure 23 involves the transport of oleate from the interface to the bulk EG subphase. The approximate the flux of oleate $\left(J_{O A}\right)$, we again applied a basic Fickian diffusion model as follows:

$$
\begin{gathered}
J_{O A}=-D \frac{\partial[O A]}{\partial z} \cong \frac{[O A]_{b}-[\mathrm{OA}]_{i}}{Z} \\
D=10^{-6} \mathrm{~cm}^{2} \cdot \mathrm{s}^{-1}
\end{gathered}
$$

We can approximate the oleate concentration at the interface $\left([\mathrm{OA}]_{\mathrm{i}}\right)$ by treating the interface as a QD monolayer. The volume occupied by a 8nm PbS QD within the ligand corona is approximately $1100 \mathrm{~nm}^{3} / \mathrm{QD}$, given an initial ligand coverage of 1000 OA/QD, we have: 


$$
\begin{aligned}
& {[O A]_{i} \sim \frac{1000 \frac{O A}{N C}}{1100 \frac{\mathrm{nm}^{3}}{N C}}=9.1 * 10^{20} \frac{O A}{\mathrm{~cm}^{3}},[O A]_{b}=0} \\
& \frac{\partial[O A]}{\partial z}=\frac{[O A]_{b}-[O A]_{i}}{Z}=\frac{-9.1 \times 10^{20} \frac{O A}{\mathrm{~cm}^{3}}}{5 \times 10^{-3} \mathrm{~cm}}=-1.6 \times 10^{23} \frac{O A}{\mathrm{~cm}^{4}} \\
& J=-D \frac{\partial[O A]}{\partial z} \sim 1.6 \times 10^{17} \frac{O A}{\mathrm{~cm}^{2} \mathrm{~S}}
\end{aligned}
$$

Note that the oleate flux determined from this model exceeds the actual removal ( $4 \times 10^{14} \mathrm{OA} \cdot \mathrm{cm}^{-2} \cdot \mathrm{s}^{-1}$, vide supra) rate by $\sim 10^{3}$. We can therefore conclude that the transport of detached ligands from the interface to the EG subphase is not the overall rate-limiting step of the process.

By process of elimination, we thus conclude that the overall oleate removal during the ORCA process is limited by the rate at which ligands are removed from the QD surface (i.e., step 3 shown in Figure 23). We note that the average ligand removal rate $(\sim 40 / \mathrm{s})$ during the first $15 \mathrm{~s}$ of the process is slower than rates that have been reported for oleyl amine ligands probed with NMR experiments by Hens. ${ }^{43}, 44$ Given that carboxylate ligands bind more strongly to the $\mathrm{PbS}$ QD surface than the corresponding amine ligands, the ligand removal rates observed in the ORCA process are consistent with expectations.

\subsection{QD surface chemistry}

Analysis the effect of ligands on QDs is critical for having a better understanding of charge transport, ${ }^{45}, 46$ optical properties $^{47}$ and assembly of QDs into ordered structures. $^{25,48}$ To study the dynamic oleate ligand chemistry during ORCA process, 
we first we adopted a model described by Roberto et al to describe the shape and composition of our $\mathrm{PbS} \mathrm{QDs}^{49}$ The $\mathrm{PbS} \mathrm{QD}$ structure is consistent with an Archimedean truncated octahedron. The particle surface is terminated by $\mathrm{Pb}$ cations, which lay at the $\{111\}$ facets and above every $S$ anion of the $\{100\}$ facets independently from the PbS core. There also binds hydroxy groups on the QD surface as displayed in Figure 20. The local photoacid generation alters the original dynamic equilibria at the surface of the polyhedral $\mathrm{PbS} \mathrm{QD}$. The thermodynamic stability and kinetic lability of ligand/core adducts is affected by ligand-ligand and ligand-subphase interactions. ${ }^{50}$ In the specific case of oleate-passivated PbS QD at surface of an EG subphase, the dynamic ligand equilibrium is also coupled to mass transport in the form of diffusion of oleic acid and lead oleate into the EG subphase.

Second, the binding energy of the oleate ligand on the $\{100\}$ is less than that on the $\{111\}$ facets ${ }^{51}$ which leads to the more pronounced ligand displacement on the $\{100\}$ facets compared to the $\{111\}$ facets of the polyhedral PbS QD core. We adopted a model demonstrated in a recent paper by Abelson et al. ${ }^{52}$ to describe the relative ligand coverage on $\{100\}$ and $\{111\}$ facets. The model of $\mathrm{PbS}$ is shown in Figure 22. The total area of per PbS QD is calculated from edge length:

$$
\text { Area_total }=6 \times a^{2}+8 \times \frac{\sqrt{3}}{4} a^{2}=(6+2 \sqrt{3}) a^{2}
$$

$\begin{array}{cc}\text { QD Diameter (d) } & 8 \mathrm{~nm} \\ \text { Edge length (a) } & 5.66 \mathrm{~nm} \\ \text { Area_100 } & 192 \mathrm{~nm}^{2} \\ \text { Area_111 } & 110.9 \mathrm{~nm}^{2} \\ \text { Area_total } & 303 \mathrm{~nm}^{2}\end{array}$

Figure 22. $\mathrm{PbS}$ QD model and surface area. 
We used FTIR spectroscopy to estimate the average number of oleate ligands bound to each QD. The FTIR spectra in Figure 23 show the results of four PbS thin films with different UV exposure time. The initial PbS QD film (prior to photoexposure) exhibits characteristic signatures of the oleate ligand covering the QD surface including asymmetric and symmetric $\mathrm{CH}_{2}$ stretches at 2921 and $2852 \mathrm{~cm}^{-1}$, a shoulder peak at $2958 \mathrm{~cm}^{-1}$ from the asymmetric $\mathrm{CH}_{3}$ stretch and a weak peak at $\sim 3004 \mathrm{~cm}^{-1}$ from the alkene $\mathrm{C}-\mathrm{H}$ of the oleate ligand. $\mathrm{PbS}$ QD films subjected to ORCA processing with varying photodosage exhibit progressively decreasing oleate ligand coverage. The extent of ligand removal can be approximately related to the integrated peak intensity in the $\mathrm{C}-\mathrm{H}$ stretching regime; this analysis shows that brief (15 s) photoexposure removes $\sim 60 \%$ of the initial ligand coverage whereas extended (120 s) photoexposure reduces the ligand coverage by approximately $90 \%$.

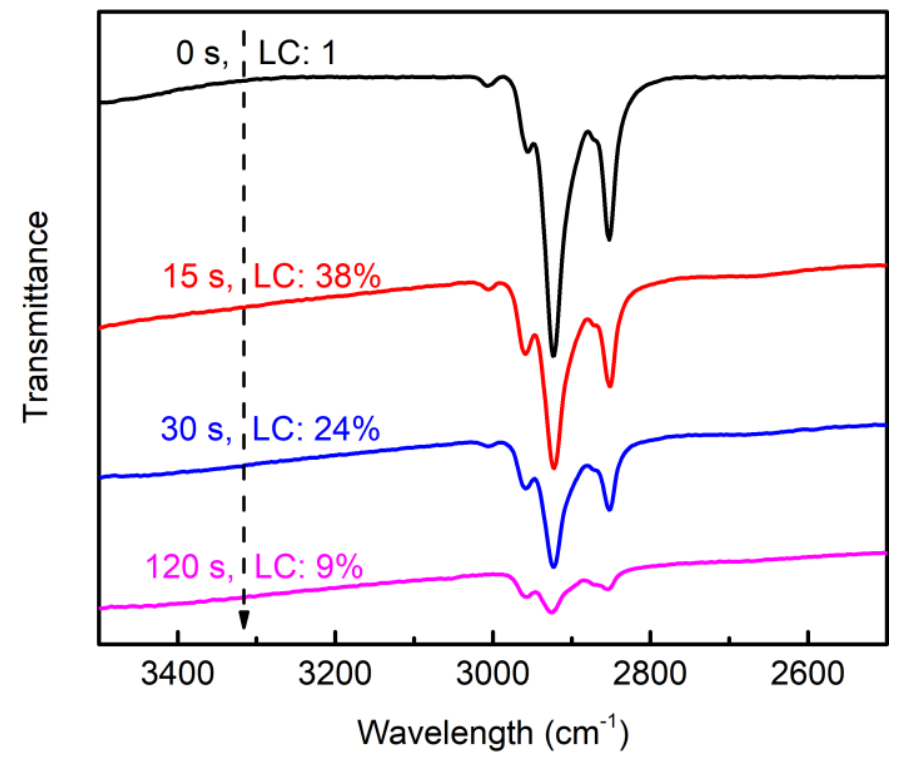

Figure 23. FTIR spectra of four different $\mathrm{PbS}$ thin films with different UV exposure time (Number shows the ligand coverage transformation). 
Based on the QD model and calculated oleate loading, we can demonstrate the relative ligand coverage on $\{111\}$ and $\{100\}$ facets as summarized in Table 4 . We converted exposure time to respective photodosage.

Table 4. Ligand coverage after certain dosage of UV exposure.

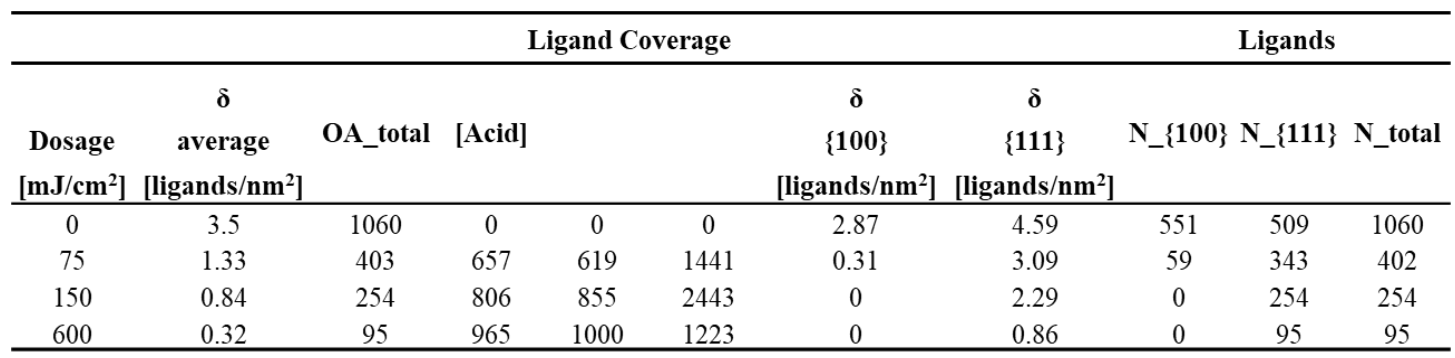

We assumed that the initial PbS QD has an averaged ligand coverage density of 3.5 ligands $/ \mathrm{nm}^{2}$. With the initial ligand coverage density, we can calculate the total numer of oleate ligands and generated acid per PbS QD after certain dosage of UV exposure. Note that as the average ligand coverage is reduced to less than $\sim 35 \%$ of the initial coverage after 30 seconds of UV exposure $\left(150 \mathrm{~mJ} / \mathrm{cm}^{2}\right)$, the $\{100\}$ facets are effectively 'bare'. With acid concentration in reaction system, we used isotherms for equilibrium coverage of oleate ligands on QD surfaces ${ }^{52}$ to determine oleate remaining ligand density and number of ligands on QDs after certain dosage of UV exposure.

$$
\begin{gathered}
\delta_{\text {avg }} \times A_{\text {total }}=\delta_{100} \times A_{100}+\delta_{111} \times A_{111} \\
N_{\text {total }}=N_{100}+N_{111}
\end{gathered}
$$

We can see more obvious ligand removal on $\{100\}$ facets from the results. The more pronounced removal of ligands from $\{100\}$ than from $\{111\}$ influences the nature of the QD-QD interaction potential which helps explain experimentally observed changes 
in the symmetry of QD assembly (Figure 24).
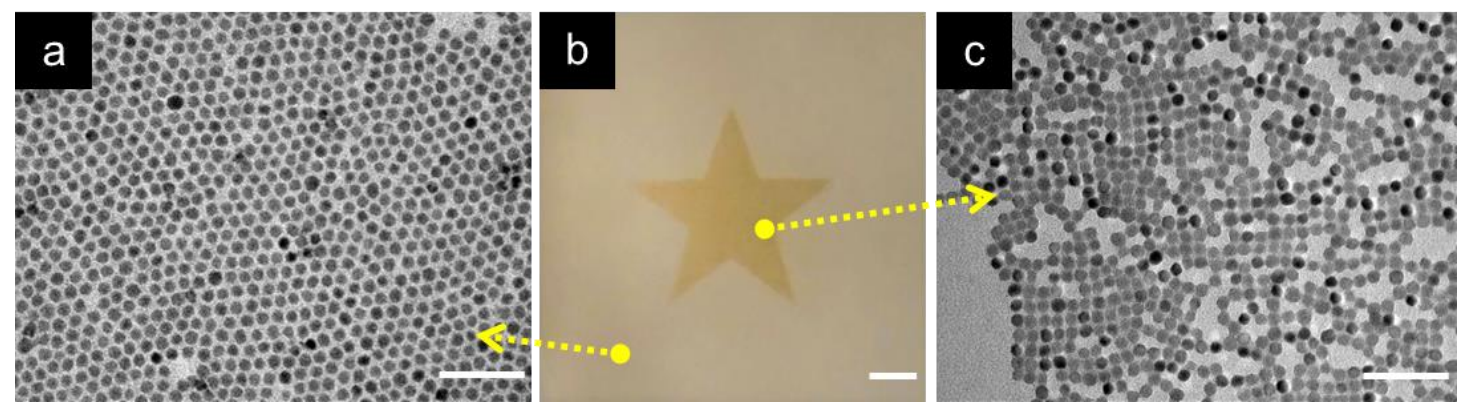

Figure 24. Digital light processing enables local definition of the superlattice structure.

(a) TEM image outside the exposed area. (b) photograph of the pattern transferred onto silicon wafer. (c) TEM image in the exposed area. Scale bars for (a) and (c) are $50 \mathrm{~nm}$. Scale bar for (b) is $1 \mathrm{~mm}$.

QD assemblies within the photoexposed regions rearrange to change in the local ordering compared to QDs in the unexposed regions. Figure 24b provides an optical photograph of the star-patterned QD film and corresponding TEM images illustrating the detailed QD structure inside and outside the photoexposed regions. The observed transformation from a hexagonally ordered assembly of isolated QDs to a predominantly square assembly of connected dots is consistent with recent reports of the formation of epitaxially connected quantum dot solids. ${ }^{16,53,54}$ This transformation involves an interesting interplay of changes in the translational QD ordering and oriented attachment. ${ }^{12}$ In previously reported systems, this transformation has been driven either by introduction of a chemical trigger or elevated temperature. ${ }^{12,17,53,55,56}$ The results in Figure 3.4a-c confirm our hypothesis that similar superlattice structure transformations can also be driven by modifying the QD ligand chemistry equilibrium 
through a PAG as the chemical trigger.

\subsection{Assembly structure control}

Beyond the ability to spatially define the regions for QD hexagonal-to-square superlattice structure transformation, the ORCA approach also provides control over the extent of the transformation. Since the structure of the QD superlattice is sensitive to the facet-specific ligand coverage on constituent polyhedral $\mathrm{QDs},{ }^{57}$ the dynamic balance of binding and desorption ligands can be adjusted by the local $\mathrm{pH}$. Both photodosage and PAG concentration provide two experimental degrees of freedom to locally tune ligand coverage and QD superlattice structure.

\subsubsection{Image analysis algorithm}

To quantitatively describe the hexagonal-to-square transformation observed in the photoexposed regions of the QD film, we analyzed the global bond order parameter, $\Psi_{n}$ with an image analysis algorithm. ${ }^{58}$ The order parameter can quantify the deviation of a specific bond order from ideal symmetry. The first step in the algorithm involves identifying the QD location and radius, which was used to convert the QD film into a set of Voronoi cells. The resulting Voronoi cells were subsequently analyzed for the symmetry by weighting angles and each vertices' lengths. Specifically, the single site $j$ with $n_{j}$ of neighbored particles for the bond order number $\mathrm{n}$ can be weighted by the length of the facet $l_{k}$ where $\mathrm{L}$ is the perimeter of the Voronoi cell enclosing site $j$. The angle of the line connecting site $j$ to site $k$ relative to an arbitrary axis is given by $\theta_{j k}$. The global order parameter can be calculated by summing $\Psi_{n j}$ for all sites. The imaginary number is given by $i=\sqrt{ }-1$. 


$$
\Psi_{j}^{n}=\sum_{k=1}^{n_{j}} \frac{l_{k}}{L} \exp \left(i n \theta_{j k}\right)
$$

$\Psi_{4}$ and $\Psi_{6}$ therefore quantify the extent of four-fold and six-fold nearest neighbor ordering within the QD assembly, respectively. Since we're interested in the transition from six-fold to four-fold symmetric regions, we have color coded the TEM data on the blue scale of $\Psi_{4}$ if $\Psi_{4}>\Psi_{6}$ and red scale of $\Psi_{6}$ if $\Psi_{4}<\Psi_{6}$. We quantified the fraction of four-fold ordering as:

$$
\frac{N_{\Psi_{4}}}{N_{\Psi_{4}}+N_{\Psi 6}}
$$

\subsubsection{Assembly structure transformation}

We used constructed algorithm to study the relationship between photodosage, PAG concentration and the superlattice structure transformation. The color maps of the bond order parameter shown in Figure 25 compare the transformation from predominantly six-fold $\left(\Psi_{6}\right)$ to four-fold $\left(\Psi_{4}\right)$ ordering of the QD films with increasing photo exposures from $0,15,30$ to 120 seconds $\left(0,75,150\right.$ and $\left.600 \mathrm{~mJ} / \mathrm{cm}^{2}\right)$. The bottom left section of each panel shows the original TEM image which provides important initial insights into the structure of QDS films. After carrying out algorithm, more 6-fold symmetry part of the superlattice shows blue $\left(\Psi_{6}>\Psi_{4}\right)$ and more 4-fold symmetry part of the superlattice shows red $\left(\Psi_{6}<\Psi_{4}\right)$. There is a clear trend that selfassembly turns from $\Psi_{6}$ symmetry to $\Psi_{4}$ symmetry correlating to exposure time. The $\Psi_{4}$ symmetry grows from less than $10 \%$ to around $60 \%$. In addition, the space between particles also becomes closer due to oleic acid removal. The correlation validates our hypothesis that self-assembly can be controlled over time by photodosage. The status 
of transition can be precisely segmented which is unachievable by traditional heat or chemical treatment. However, there seems to be a maximum interval close to $60 \%$ of $\Psi_{4}$ symmetry. The same phenomenon was also observed in the study of PAG concentration effect on structure transformation. Figure 26 below summarizes the effect of varying PAG concentration at fixed photodosage. We fixed the exposure time at $30 \mathrm{~s}\left(150 \mathrm{~mJ} / \mathrm{cm}^{2}\right)$ and varied the concentration from $0.25 \mathrm{~g} / \mathrm{L}$ to $2 \mathrm{~g} / \mathrm{L}$. The $\Psi_{4}$ symmetry grows from around $28 \%$ to around $60 \%$. In the condition of high PAG concentration $(2 \mathrm{~g} / \mathrm{L})$, particles were dissolved by continuously released acid and the edge can be barely seen (Figure 26d).

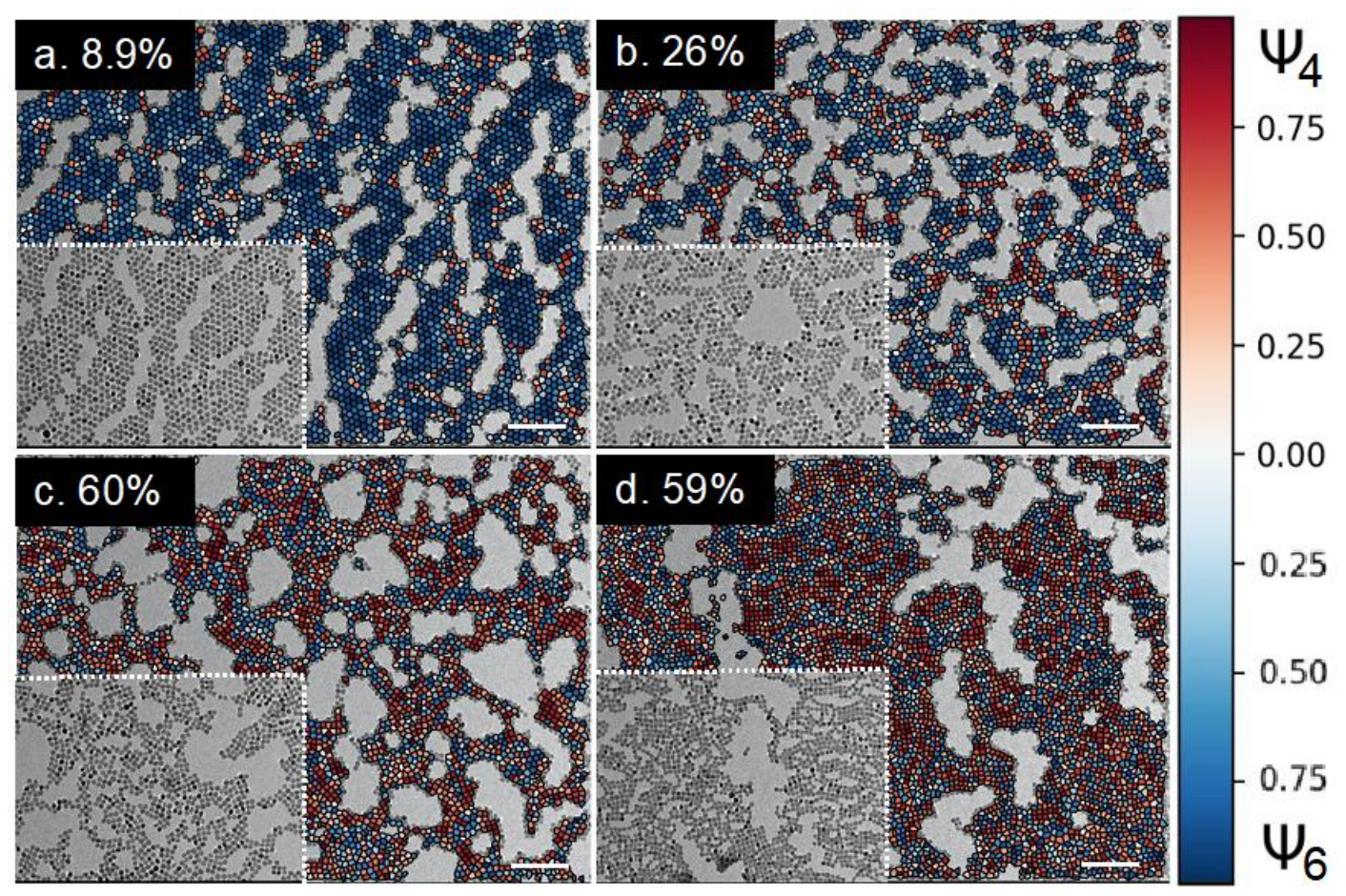

Figure 25. Symmetry transformation with different light dosage. The bottom left section of each panel shows the original TEM image and the rest of the panel shows the Voronoi cells color coded according to the local bond ordering parameter (blue scale of $\Psi_{6}$ if $\Psi_{6}>\Psi_{4}$ and red scale of $\Psi_{4}$ if $\Psi_{6}<\Psi_{4}$ ). (a) 0 s with $8.9 \% \Psi_{4}$ symmetry. 
(b) $15 \mathrm{~s}$ with $26 \% \Psi_{4}$ symmetry. (c) $30 \mathrm{~s}$ with $60 \% \Psi_{4}$ symmetry. (d) $120 \mathrm{~s}$ with $59 \%$

$\Psi_{4}$ symmetry. Scale bars for a-d are $100 \mathrm{~nm}$.
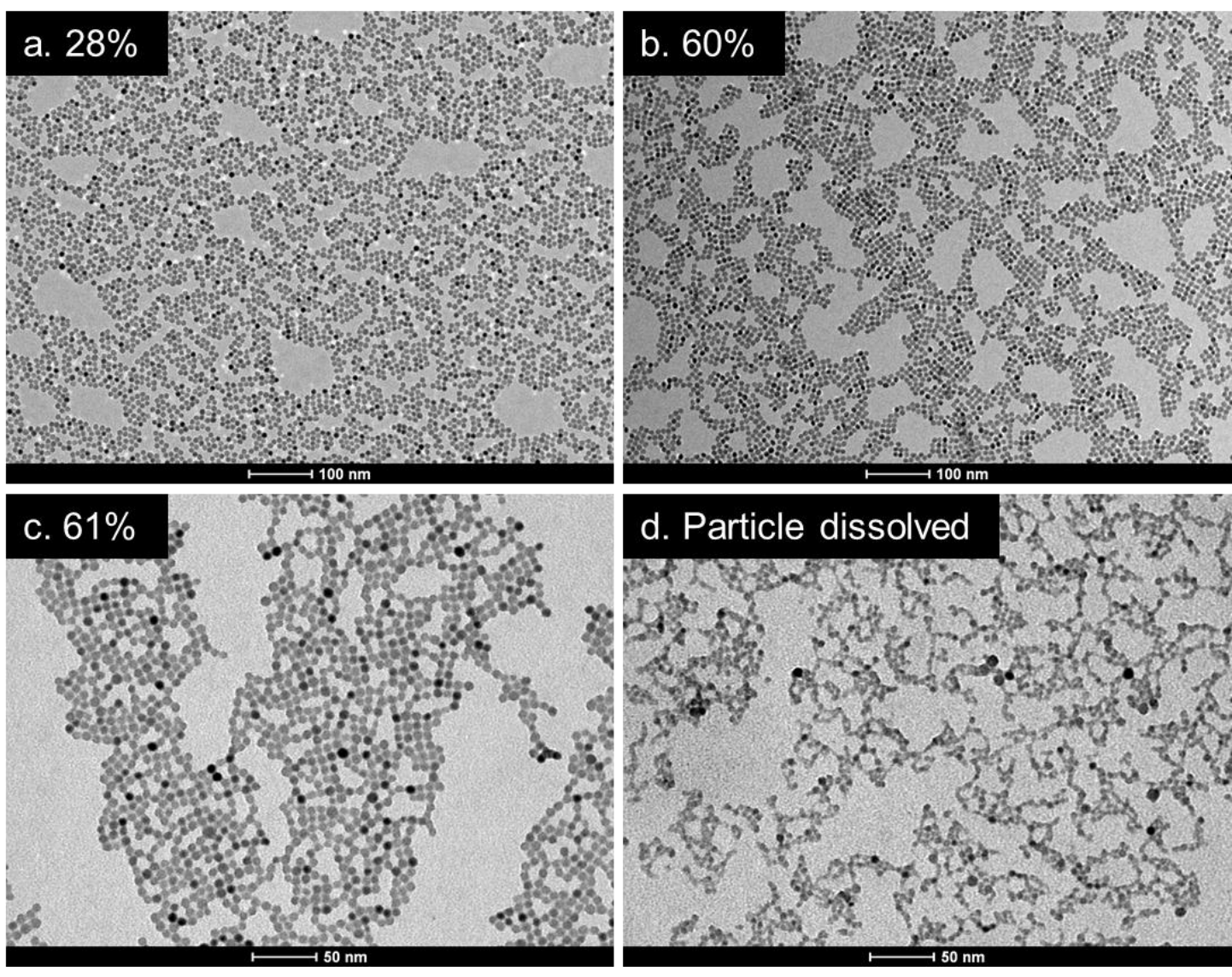

Figure 26. Symmetry transformation with different PAG concentration. (blue scale of

$\Psi_{6}$ if $\Psi_{6}>\Psi_{4}$ and red scale of $\Psi_{4}$ if $\Psi_{6}<\Psi_{4}$ ). (a) $0.25 \mathrm{~g} / \mathrm{L}$ with $28 \% \Psi_{4}$ symmetry. (b)

$0.5 \mathrm{~g} / \mathrm{L}$ with $60 \% \Psi_{4}$ symmetry. (c) $1 \mathrm{~g} / \mathrm{L}$ with $61 \% \Psi_{4}$ symmetry. (d) $2 \mathrm{~g} / \mathrm{L}$. Scale bars for $\mathrm{a}$ and $\mathrm{b}$ are $100 \mathrm{~nm}, \mathrm{c}$ and $\mathrm{d}$ are $50 \mathrm{~nm}$.

\subsubsection{Surface chemistry involved structure transformation}

As we analyzed above, ORCA process shows the outstanding ability to control superlattice structure transformations that driven by modifying the QD ligand chemistry equilibrium through a PAG as the chemical trigger. The oriented attachment of PbS QDs can be understood in context of selective ligand displacement from the 
$\{100\}$ facets of the polyhedral shaped inorganic PbS QD core. The displacement of oleate ligands is more pronounced from $\{100\}$ than from $\{111\}$ facets which impacts the sphericity of the QDs and the interaction between them at the fluid interface. Whereas ligand shell of the initial (i.e., fully ligated) QDs is approximately spherical, ligand deprotection from specific facets leads to a ligand shell with an effective sphericity that more closely reflects the polyhedral (i.e., truncated cube) shape of the inorganic QD core. The change in the effective sphericity of the QD ligand shell drives the $\Psi_{6}$-to- $\Psi_{4}$ structure transformation of the superlattice and ultimately leads to the directed attachment of mutually exposed $\{100\}$ facets of proximate QDs in the film. To provide a mechanistic picture of how the photogenerated acid impacts the QD ligand chemistry and QD film structure transformation it is important to recognize that the ligand chemistry of colloidal QDs is in dynamic equilibrium with the surrounding fluid. ${ }^{49}, 59$ Figure 21 illustrates the dynamic equilibrium between oleate bound to the $\mathrm{PbS}$ NC surface and lead oleate as well as oleic acid in the surrounding fluid. ${ }^{56}$ The photogenerated acid influences the thermodynamic stability and kinetic lability of the oleate/PbS QD core adduct at the fluid surface. Extended photodosage progressively lowers the $\mathrm{pH}$ near the fluid surface which shifts the equilibrium towards the lead oleate and oleic acid in the surrounding fluids. Both moieties subsequently diffuse from the interface into the bulk EG subphase. However, high PAG concentration lowers the surrounding $\mathrm{pH}$ too much that destructed the PbS QD layers (Figure 26d).

\subsection{QD size and interparticle spacing reduction}

As shown in Figure 21, the ligand is displaced in the form of oleic acid and lead oleate. 
The removal ligands in the form of lead oleate results in a small reduction of the QD core size. Besides, the releasing proton forms an acidic atmosphere in the solution which would also etch the $\mathrm{PbS}$ core and cause size reduction. ${ }^{40}$ And the etching phenomenon would be more notable as the $\mathrm{Ph}_{2} \mathrm{I}$-pyranine solution becomes more concentrated. As shown in Figure 26d, the PbS QDs seem to be melted in case of high concentration, $2 \mathrm{~g} / \mathrm{L}, \mathrm{Ph}_{2} \mathrm{I}$-pyranine.

We can monitor size changes of $\mathrm{PbS}$ QDs on the basis of size-dependent optical properties $^{36}$. UV-vis absorption spectroscopy is an efficient technique to probe the size-dependent optical properties of QDs. Figure 27 shows the measured Near infrared (NIR) transmission spectra of four PbS QD films with different UV exposure time. We observed that exposing to UV light caused slight blueshift and the blueshift is more obvious with the increasing UV exposure time. The small blue shift in the excitonic peak proves the size reduction.

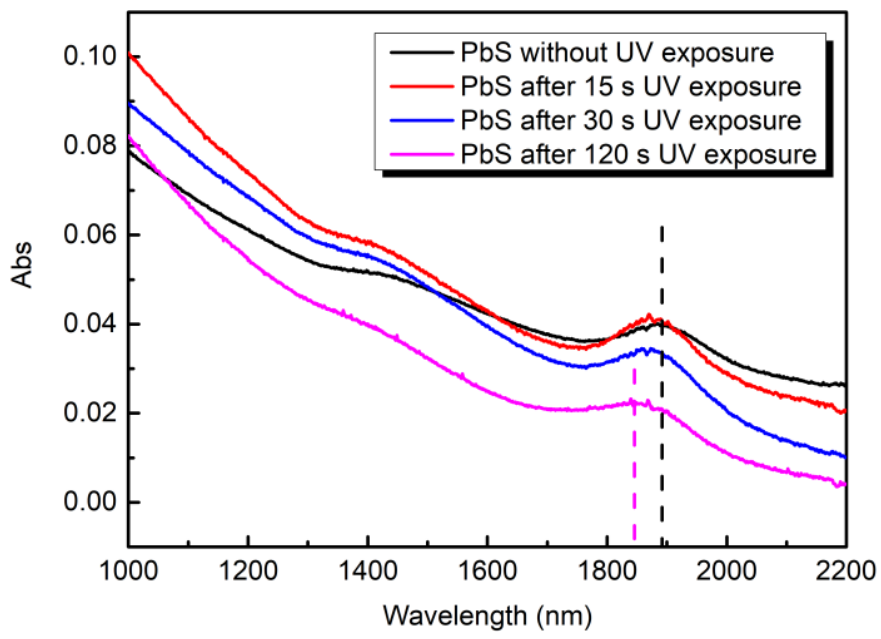

Figure 27. NIR absorption spectra.

We also did statistical analysis of TEM images (same batch with Figure 25) performed by using image analysis software packages, ImageJ. The results are demonstrated in 
Figure 28 (black dot curve) which shows a trend of size reduction.

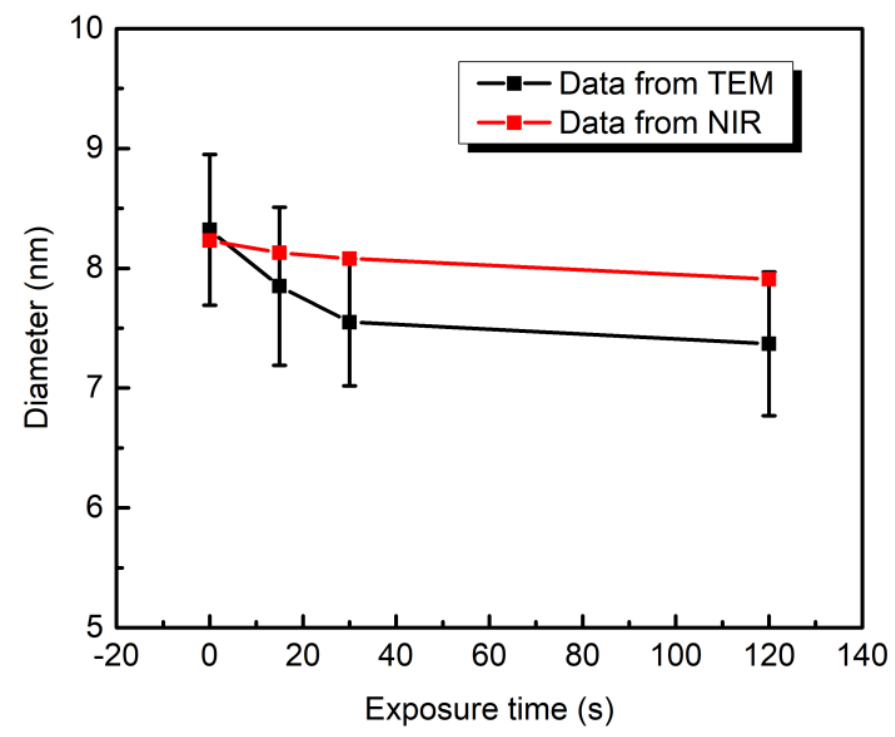

Figure 28. Comparison of QD size obtained from NIR measurements and TEM images.

We also collected size information from NIR transmission spectra (red dot curve) by a polynomial fit. They both clearly show a trend of size reduction, however, there's an interesting deviation between NIR transmission spectra and TEM results. Apart from the original particle size, with the raising UV exposure time, the particle size inferred from the NIR transmission spectra is significantly larger than the QD diameter collected from TEM. The QD size derived from NIR transmission spectra is larger than the particle size inferred from TEM. The reason causes to the bias between NIR and TEM results is that measured NIR transmission spectra do not reflect the average QD size but the largest QDs in attached films. ${ }^{21}$

As a further evidence, we obtained the radial distribution function (RDF) based on QD locations directly collected from TEM images (Figure 25). In general, RDF is a 
measure of the probability of finding a particle at a distance of $r$ in a shell $\mathrm{dr}$ away from a given reference particle, relative to that for an ideal gas. ${ }^{60}$ Due to its intuitive nature, we can get significant insight of nanoparticle structure from the analysis of RDF including interparticle distance, assembly symmetry and packing disorder. We compared RDF of two PbS QD thin films, original film and film after ORCA process (120 s). The results are reported in Figure 29 and more comparison are shown in Figure S3.

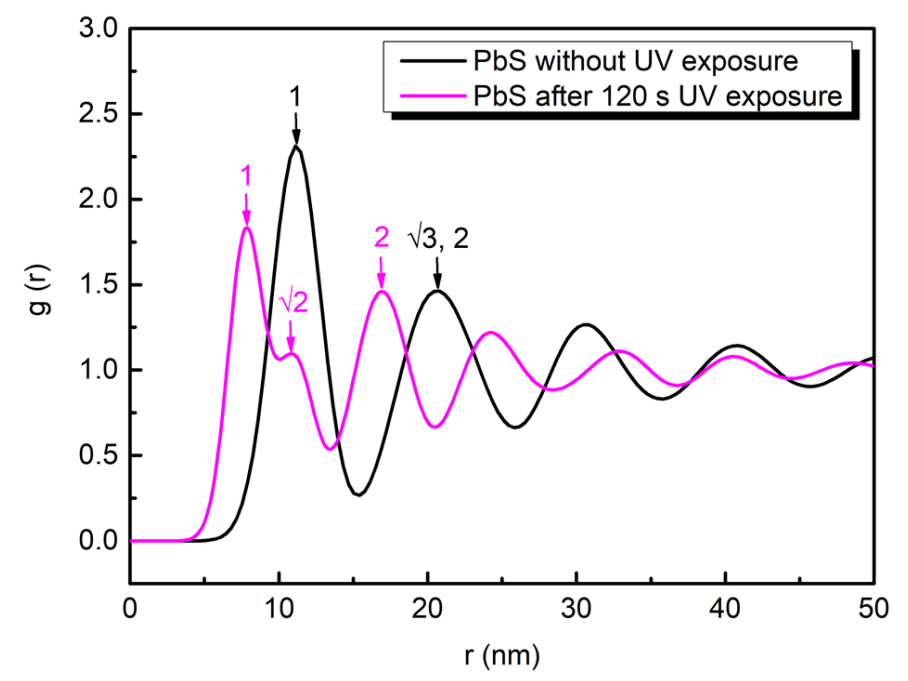

Figure 29. Radial distribution function analysis of TEM images.

The first peak of the RDF reflects the center-to-center spacing which is reduced from $11.1 \mathrm{~nm}$ in the initial PbS QD film to around $7.9 \mathrm{~nm}$ in the QD film subjected to a 600 $\mathrm{mJ} / \mathrm{cm}^{2}(120 \mathrm{~s})$ photodosage. It is also a prove of significant reduction in inter-QD spacing due to reduced ligand coverage. The RDF analysis also corroborates the change in QD superlattice structure. The initial film shows a broad second order peak incorporating both ' $\sqrt{3}$ ' and ' 2 ' peaks of a hexagonal lattice whereas the ORCA treated 
film shows a higher order peak at $10.8 \mathrm{~nm}$ corresponding to the expected ' $\sqrt{2}$ ' peak of a square lattice.

\subsection{Electric conductivity}

The reduced interparticle spacing that results from removing long hydrocarbon molecules would facilitate strong electronic coupling between neighboring QDs. ${ }^{61}$ As discussed in Figure 28, estimating the effective particles size from the absorption peaks, and comparing it to the value obtained from TEM images show a consistent, growing mismatch with increasing exposure. This suggests an increasing electronic coupling between the neighboring QDs. To analyze the effect of ORCA process on electronic properties, we measured two-terminal conductivity of PbS QD films with varied light dosage (Drop cast PbS, 0 s UV exposure, 15 s UV exposure, $30 \mathrm{~s} \mathrm{UV}$ exposure and $60 \mathrm{~s}$ UV exposure). The output characteristics are reported in Figure 30.

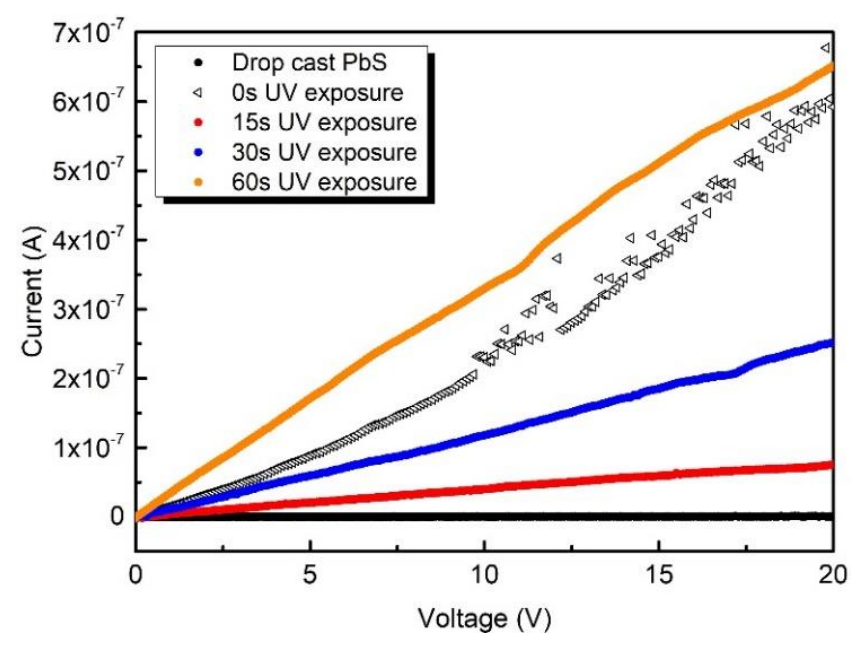

Figure 30. ID-VD (output) characteristics of five devices.

The results of drop cast device and 15s, 30s, 60s devices show the ORCA process 
enhances the electronic conductivity; increasing conductance suggests that electronic coupling between QDs in the film increases with increasing photodosage. However, we note that the 0s device (i.e., control QD film assembled at fluid interface without photoexposure) also showed improved conductivity compared to the drop-cast control sample. Since the unexposed film cannot be washed with toluene, we attribute this enhanced conductivity to residual EG in the unexposed QD film. Accordingly, we could not compare this device with other exposure devices because of uncontrolled variables. Consequently, we compared calculated resistivity, $\rho$, of drop cast devices and devices after ORCA process (15s, 30s, 60s).

$$
\rho=R \cdot S / L
$$

$\mathrm{R}$ is resistance of corresponding devices and $\mathrm{L}$ equals to $5 \mu \mathrm{m}$ here. The measured QD thin film channel is around $75 \mathrm{~nm}$, so the cross-sectional area $\mathrm{S}$ is $7.5 \times 10^{-10} \mathrm{~m}^{2}$.

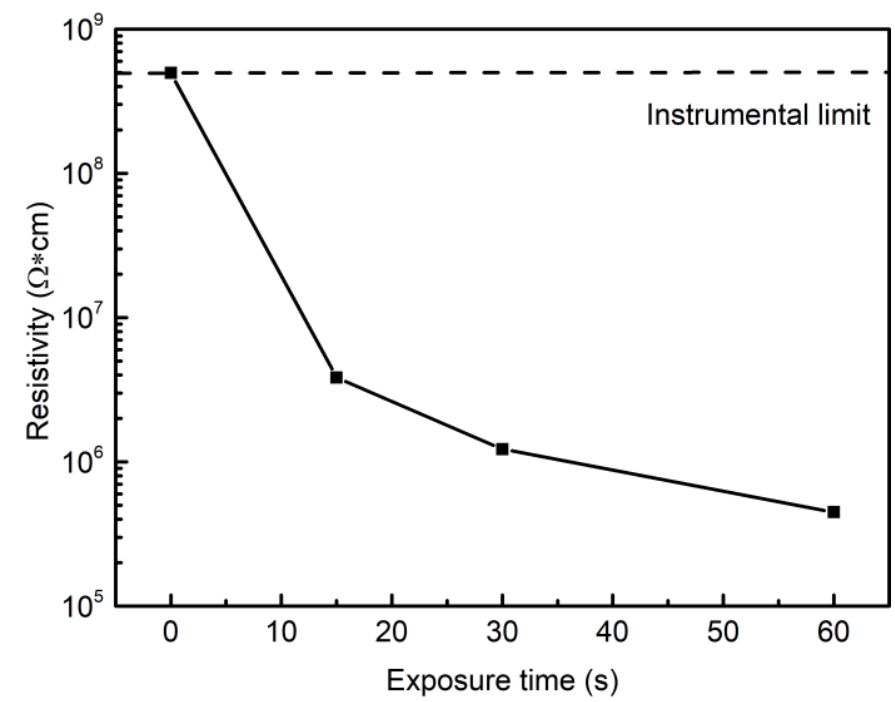

Figure 31. Resistivity of $\mathrm{PbS}$ thin films with different light dosage.

Figure 31 summarizes the trend in reduced resistivity with increasing photodosage 
treatment. We can relate extent of ORCA process with superlattice spacing and structure change and charge transport enhancement.

\subsection{Pattern printing}

The ORCA process enables directly light activated patterning of inorganic nanocrystals at a liquid interface. With the digital light projector (DLP), we can shine a light with a certain shape on the liquid-liquid interface. The formed pattern at the interface is visible to eye due to a darker color than the surrounding area as shown in Figure 24. In the exposed area, the nanoparticles undergo the ORCA process, the original ligand on the particles is peeled off and forms an epitaxially connected solid. In contrast, in unexposed areas, there are still unconnected particles with long hydrocarbon ligands. We then estimated the resolution of the printed patterns. In this project, we tried $\mathrm{PbS}$ and $\mathrm{CdSe}$ QDs as ink.

\subsubsection{Positive pattern and negative pattern}

As discussed in section 3.1, because of the large excess of the monoanion of ethylene glycol at the fluid interface, we assume that the 2-hydroxyethanolate bind to freshly vacated sites on the QD surface. The strong hydroxide vibrational signal in FTIR spectra of the photoexposed QD film (Figure 32) proved our assumption. The sample with $60 \mathrm{~s}$ of UV exposure was cleaned by toluene and put in the $85{ }^{\circ} \mathrm{C}$ Muffle furnace

to remove remaining $\mathrm{EG}$. The strong $\mathrm{O}-\mathrm{H}$ stretch in spectrum is from 2hydroxyethanolate bind to the QD surface. 


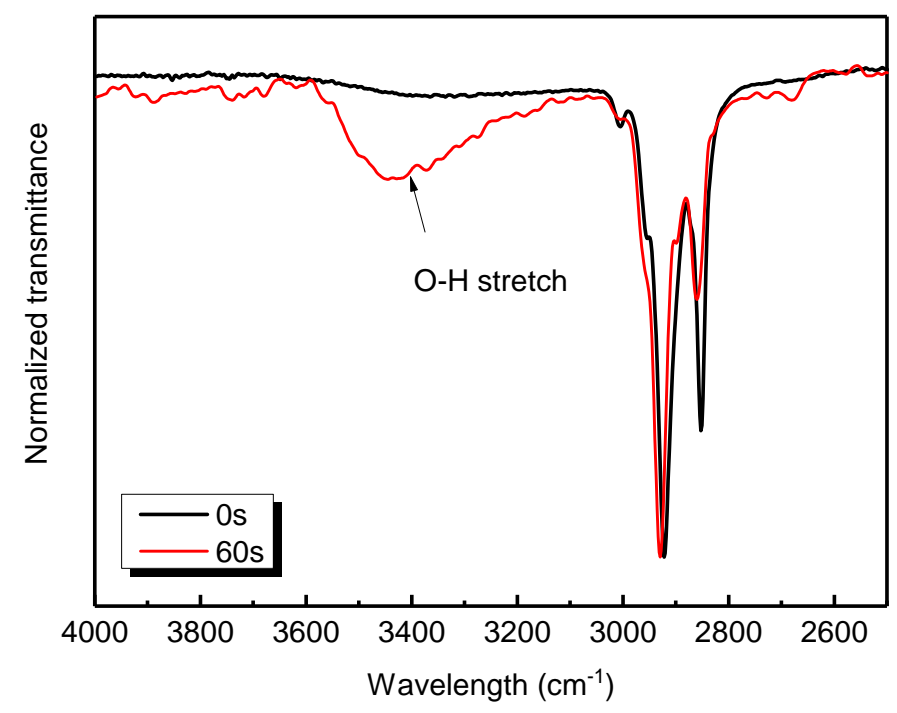

Figure 32. FTIR spectra of $\mathrm{PbS}$ film at $0 \mathrm{~s}$ and $60 \mathrm{~s}$ of UV exposure.

The desorption of original oleate ligand and small amount of replacement of 2hydroxyethanolate on QD surface changes the solubility of QDs in the reaction system; the photoexposed regions are soluble in polar solvents and the unexposed regions are soluble in nonpolar solvents. Therefore, we could get two kinds of patterns with different solvents to wash the QD thin films after transfering. We can efectively creat a negative pattern by using methanol as shown in Figure 33a. Conversely, positive patterns can be formed by washing the photoexposed and transferred QD films with non-polar solvents like toluene as shown in Figure 33b. We fabricated a thicker $\mathrm{PbS}$ film (therotically 10 layers) and cleaned it with methanol. The enlarged film in microscope shows the details of negative patterns in Figure S2. 

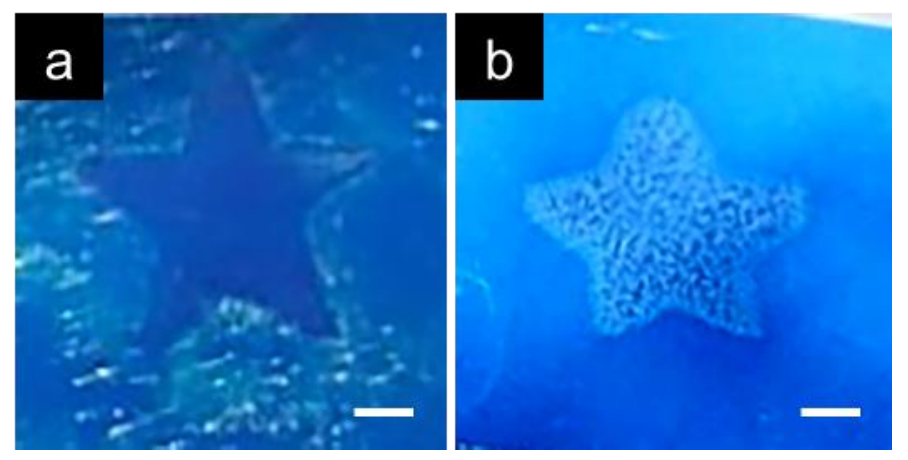

Figure 33. a. Negative pattern on silicon wafer. b. Positive pattern on silicon wafer.

Scale bars: $1 \mathrm{~mm}$.

\subsubsection{Resolution of printed patterns}

Resolution is an important parameter to evaluate the quality of printed patterns. As shown in Figure 33b, the resolution of positive pattern is unsatisfactory. Lots of cracks shows inside the pattern and the star gets out of shape. Since ORCA process involves three operation steps including introducing QD thin films at liquid-liquid interface, UV exposure and transferring QD thin films, changes from every step would influence the resolution of printed patterns. With controlled introducing and transferring steps, we fabricated a designed pattern with different photodosage to study the effect of photodosage on printing resolution. Patterns a-c show in Figure 34 are three negative patterns with different light dosage (15 s, $30 \mathrm{~s}$ and $120 \mathrm{~s})$. Figure $34 \mathrm{~d}-\mathrm{f}$ are the enlarged views under microscope for Figure 34a-c. Pattern with 120 s of UV exposure presents the best resolution, however the edge of square becomes crooked. Although in our study, ORCA process does not show comparable resolution with traditional photolithography, the ability to control assembly structure is extraordinary. Thus, one of our future direction is to modify the printing resolution of ORCA process. Figure 35 shows the other printed patterns on silica wafer with PbS or CdSe QDs. 


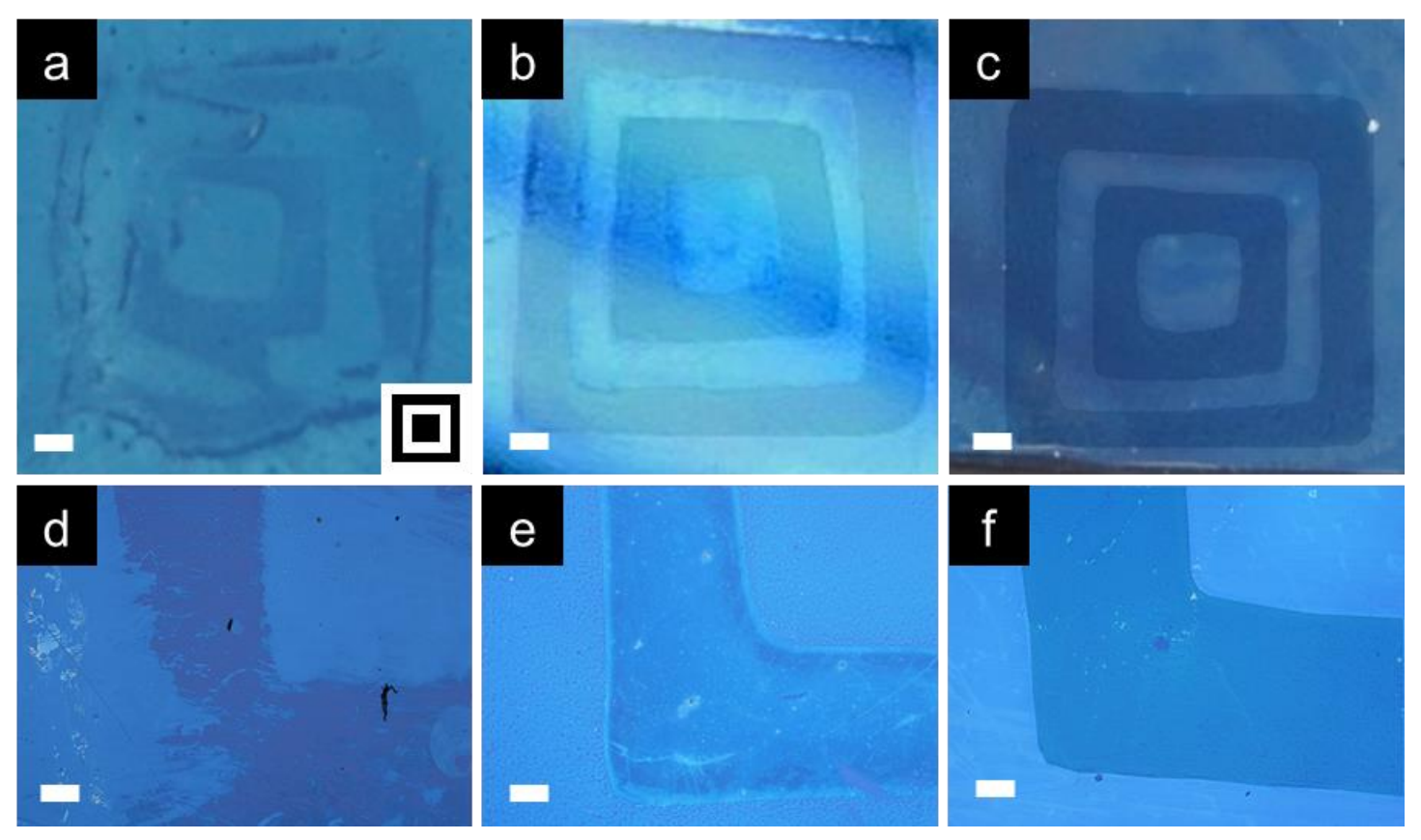

Figure 34. Printed pattern on silica wafer with different photodosage. a. 15 s. b. 30 s.

c. $120 \mathrm{~s}$. Bottom right section in a is the original exposed pattern for $\mathrm{a}, \mathrm{b}$ and c. d-f are cooresponding enlarged views of a-c. Scale bars for a-c are $1 \mathrm{~mm}$, d-f are $0.2 \mathrm{~mm}$.
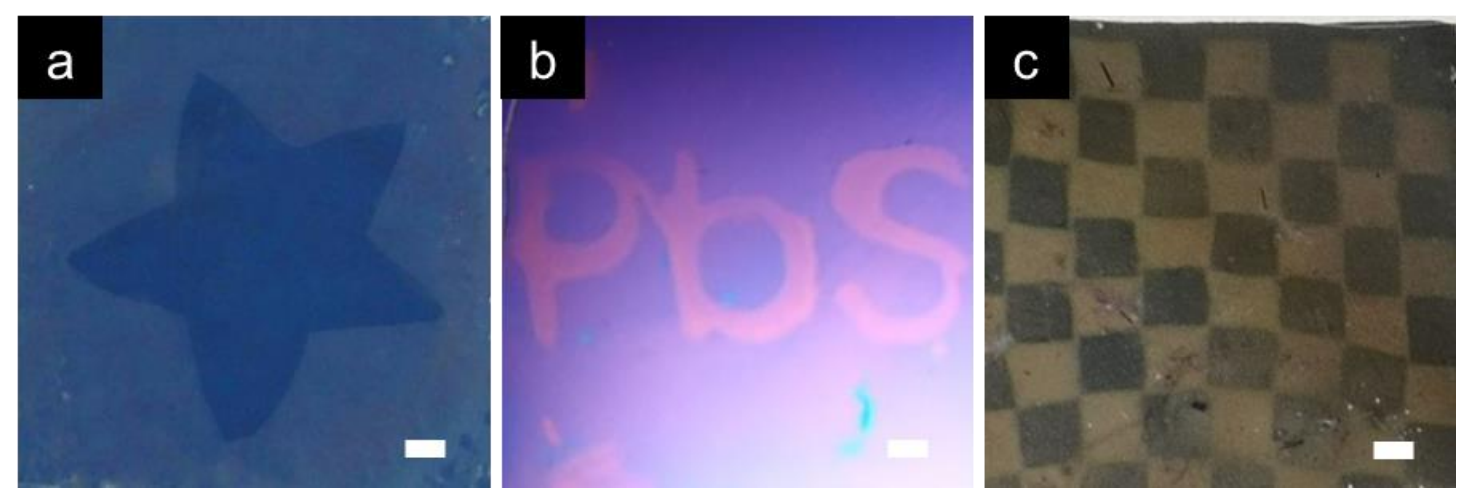

Figure 35. Printed paterns on silica wafer with $\mathrm{PbS}$ or CdSe QDs. a. Star (CdSe). b. $\mathrm{PbS}(\mathrm{PbS})$. c. Checkerboard (PbS). 


\section{Chapter 4 Summary and outlook}

The subject of this thesis is a study of photoinitiated transformation of nanocrystal superlattice polymorphs assembled at a fluid interface with four directions: mechanism of chemistry, macroscale study, microscale study and study of properties. In this chapter we summarize the main conclusions of the work and discuss future perspectives. The general conclusions, especially regarding the use of experimental methods are described first. Secondly, specific conclusions relating the four general directions of the thesis are set out.

\section{1 summary}

This work demonstrates a photochemistry assisted assembly of inorganic nanocrystals called optically-driven reorganization of colloidal assemblies (ORCA). Based on the knowledge established from QD self-assembly at fluid interfaces and direct lithography of inorganic nanocrystals, we designed the three-step procedure of ORCA

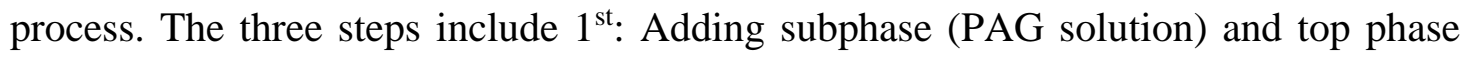

(QD solution); $2^{\text {nd }}$ : UV exposure with covered quartz and $3^{\text {rd. }}$ Transferring. The 
concentration range of PAG solution and $\mathrm{PbS}$ QD solution was decided by detailed experiments. The systematically defined ORCA process shows how QD assembly at the surface of a fluid subphase can be combined with photolithographic techniques to locally control structure transformations in the QD film and print defined 2D patterns within millimeter scale. Photolysis of the PAG within the exposed regions shifts the dynamic equilibrium of the oleate/PbS QD core adduct to reduce ligand coverage. On the one hand, the change of ligand coverage enables ORCA process to printed two kinds of 2D pattens, positive and negative. On the other hand, due to the polyhedral QD shape and facet-specific ligand coverage, the ligand removal is accompanied by a change in the effective sphericity of the ligand shell which drives a change in the QD superlattice structure from predominantly six-fold to four-fold. The extent of this transformation is related to the dosage of the photoexposure which enables us to control the QD superlattice structure via photodosage. Besides, in the two-terminal conductivity experiments, due to the ligand removal and decreasing interparticle distance the electrical conductivity is shown to enhance in higher four-fold symmetry superlattice. This work underscores the potential of applying photochemistry with selfassembly as the guidance for rational design in self-assembled superstructures.

\subsection{Outlook}

For future work, four main topics might be of high interest:

\subsubsection{Improvement of printing resolution of ORCA process}

First, future works should, in general, be devoted to the study of the resolution improvement and limit of ORCA process. Since ORCA process involved three steps, 
first, the parameters of every step would influence the overall resolution, we have to find out the resolution limiting step or limiting parameter. We get some insight from the observations shown in Figure 36. The black dash line in each panel presents the original edge of printed star, and the yellow dash line presents the extended edge after different sitting time on liquid surface. The edge of star became more extended with longer sitting period. We assumed the main factor that contributes to this phenomenon is PAG lateral diffusion. Considering the complex diffusion condition on the liquid surface as shown in Figure 37, which involves subphase solution EG and QD solid, more detailed investigations are required.
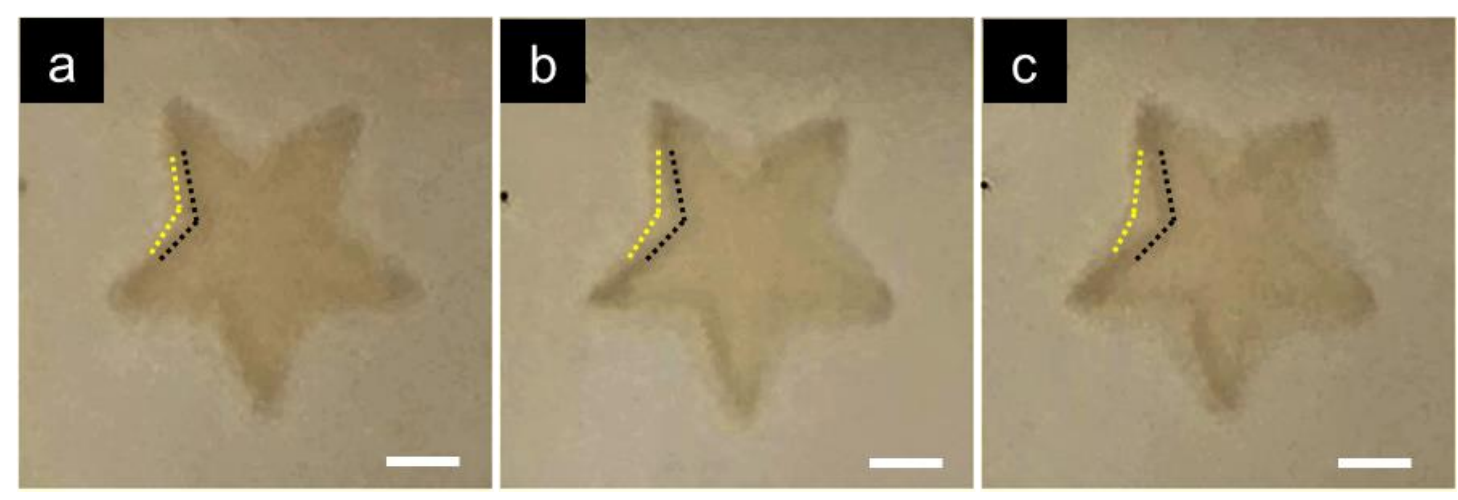

Figure 36. Printed star at the liquid surface after $30 \mathrm{~s}$ of UV exposure with different overall sitting time (Black dash line presents original printed edge and yellow dash line presents extended edge after certain siting time), a. 3 mins. b. 5 mins. c. 7 mins. Scale bars for a-c are $1 \mathrm{~mm}$. 


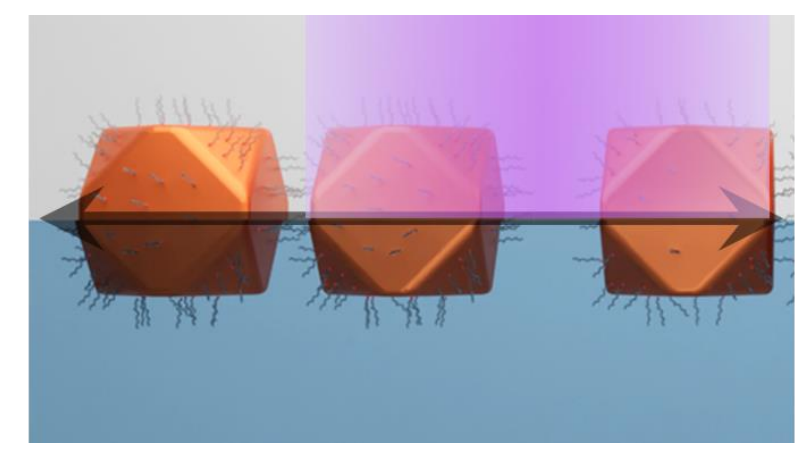

Figure 37. Diffusion condition on the liquid surface.

Besides, it is assumed that the QD mobility on the fluid interface is of highly relevance of the bad resolution we obtained in the experiments. The transferring step would be suffered most by high QD mobility on the liquid surface.

\subsubsection{Build photochemistry library for ORCA process}

As we have discussed in Chapter 3.1.1, the rate limiting step is the ligand displacement on NC surface. Although the ligand removal rates observed in the ORCA process are consistent with expectations, we are thinking about trying other photoacid generators. Thus, it inspired us a future work direction, to build a photochemistry library for ORCA process. 


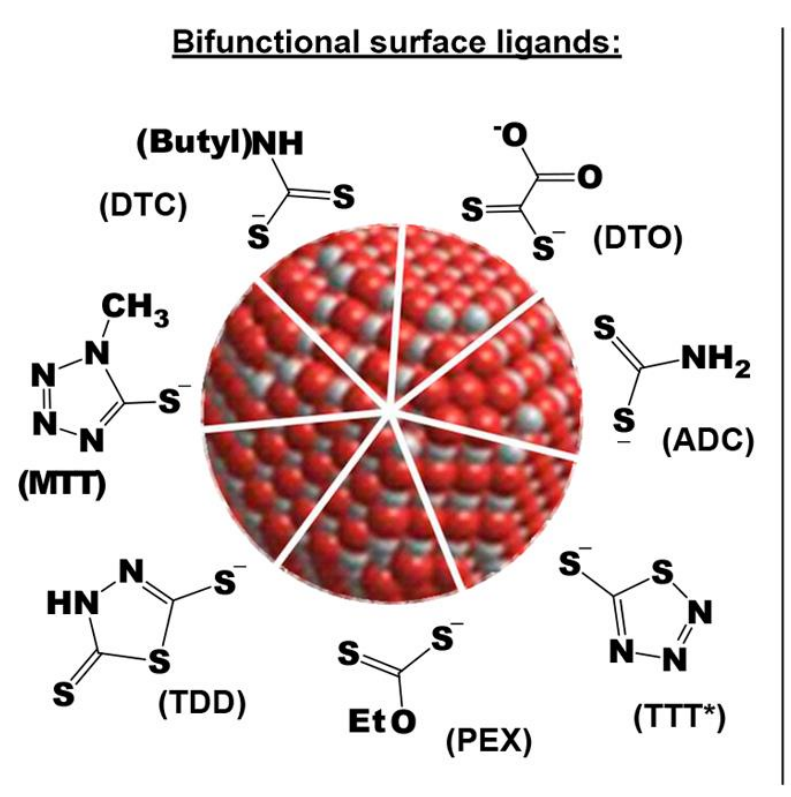

\section{Photochemically active additives:}
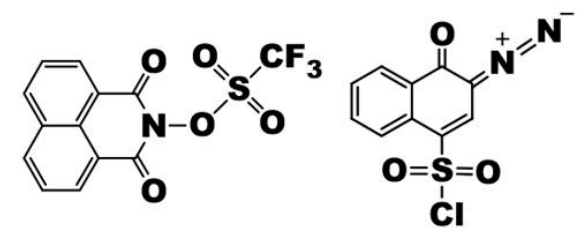

(HNT)

(DNQ)

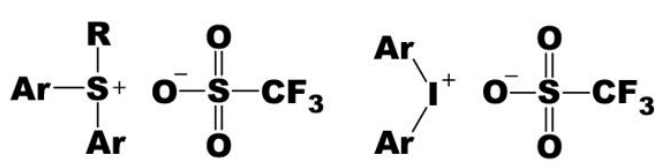

(AST*)

(AIT*)

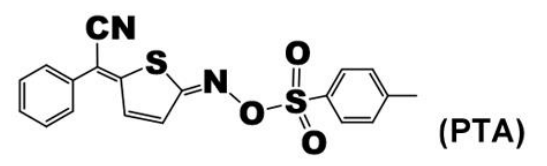

Figure 38. Chemical Structures of Designed Photoactive Ligands Used for DOLFIN ${ }^{62}$

With reference to a new work of Wang et al. (Figure 38), we can design a working chemistry system for ORCA process.

\subsubsection{Layer-by-layer multicomponent of ORCA process}

Layer-by-layer (LbL) assembly provides a versatile tool for engineering materials. There are numerous applications of LbL. It can be used on functional coatings such as coatings for photonics and energy-related applications such as switchable coatings for photoelectrochemistry. ${ }^{63}$ It also has biological applications such as encapsulation platforms for nanodelivery. ${ }^{64,65}$ A further point of interest might be combining ORCA process with $\mathrm{LbL}$ assembly to enable multicomponent superstructures. Figure 38 presents the perspective experimental process of LbL assembly of ORCA. Comparing with ORCA, we can fabricate 3D multilayer nanostructures with higher complexity. We can also tune the properties for the 3D nanostructures by altering nano building blocks for different layers. Novel and enhanced properties might be created through 
designing the shape and thickness of each layer.
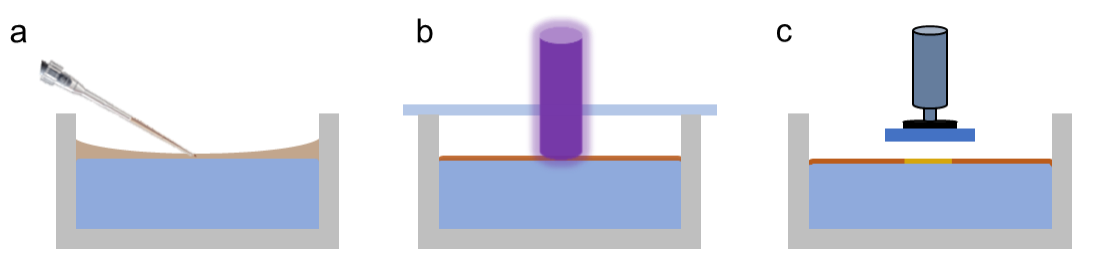

d

Figure 39. Experimental process of LbL assembly of ORCA. a. Adding subphase and top phase. b. UV exposure with covered quartz. c. Transferring. d. LbL atachment.

\subsubsection{ORCA process with two-photon lithography}

Two-photon lithography is a powerful tool to create complex, small-scale 3D structures via polymerization. By focusing a femtosecond laser into photoresist, polymerization can be locally induced within the focal region of the beam. And 3D polymer structures with any virtually geometry can be created by rastering the laser focus throughout the photoresist in three dimensions. Greer's group developed a lithography-based process to create complex 3D nano-architected metals with high resolution. ${ }^{66,67}$ They synthesized hybrid organic-inorganic materials that contain $\mathrm{Ni}$ clusters as a metal-rich photoresist, then use two-photon lithography to sculpt 3D polymer scaffolds, and pyrolyze them to volatilize the organics and finally form the target 3D structure and obtain high resolution. If we exchange the photoresist with materials used in ORCA as shown in Figure 39, we can enable 3D printing of inorganic QDs without polymerization and directly device fabrication. 

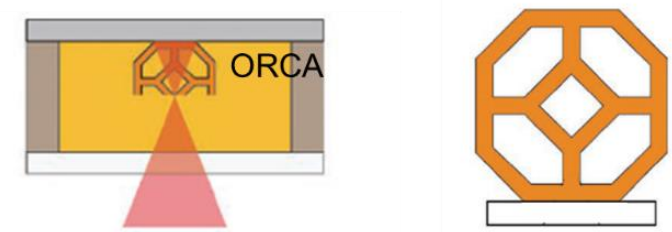

Two-photon lithography

Figure 40. Process for 3D printing of inorganic nanocrystals. 


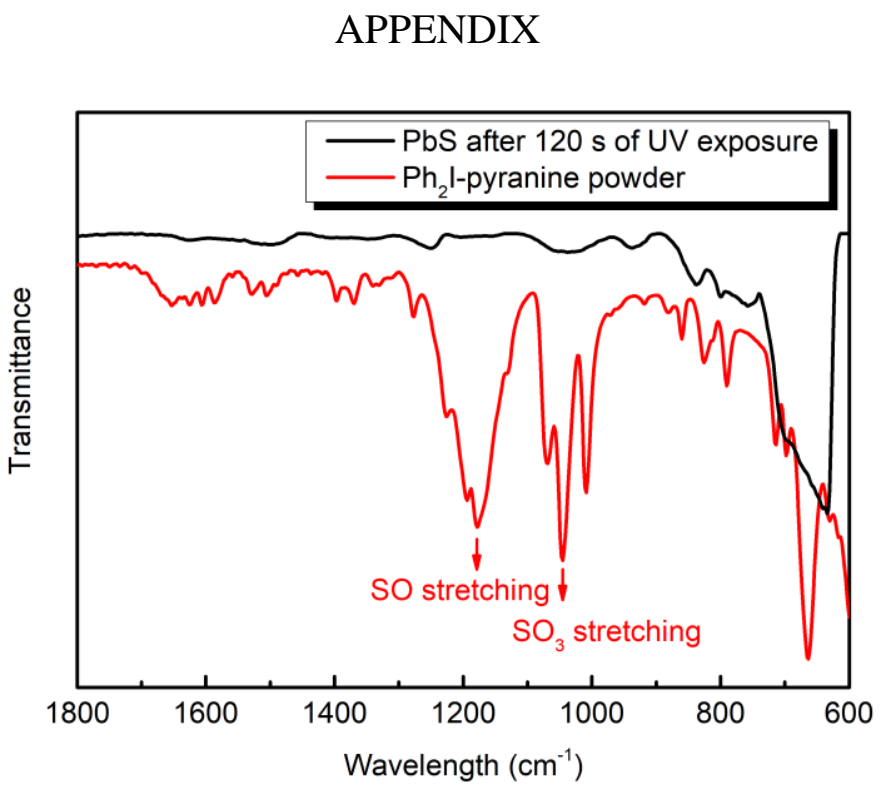

Figure S1. FTIR spectra of PbS QD thin films after $120 \mathrm{~s}$ of UV exposure and $\mathrm{Ph}_{2} \mathrm{I}-$ pyranine
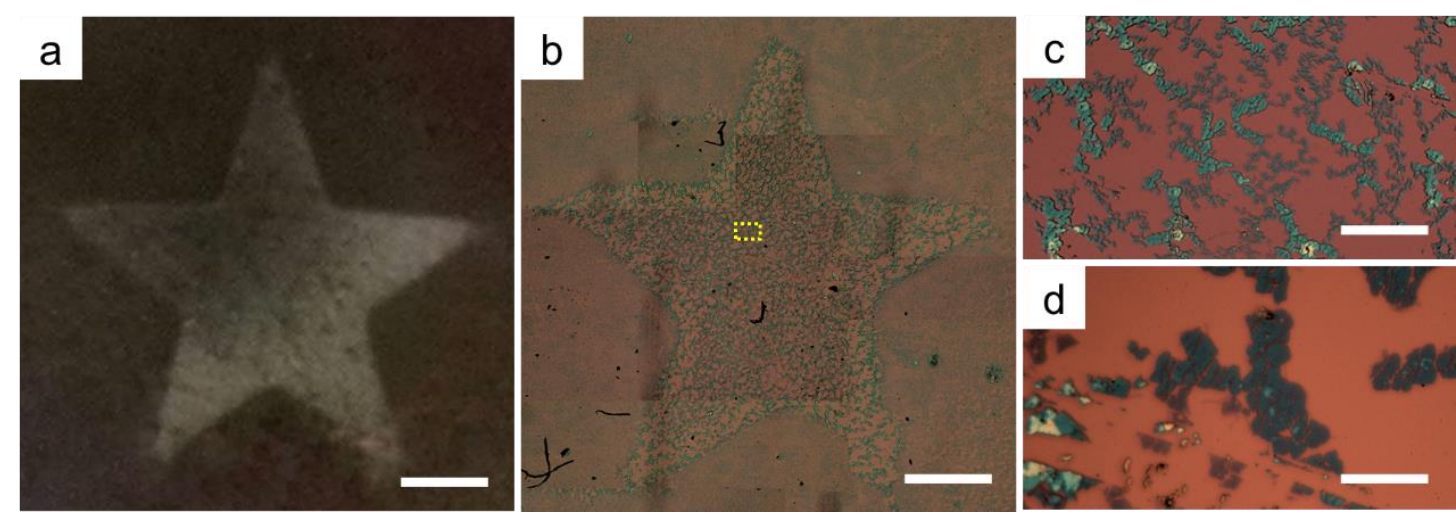

Figure S2. Negative pattern of a thick PbS QD film. a. Negative pattern on silica wafer. b. Negative pattern under microscope. c. Large version of film in the yellow dash-line box. d. Large version of film in the yellow dash-line box. Darker areas are empty. Scale bars: a and b are $1 \mathrm{~mm}$, c is $0.1 \mathrm{~mm}$ and $\mathrm{d}$ is $0.05 \mathrm{~mm}$. 


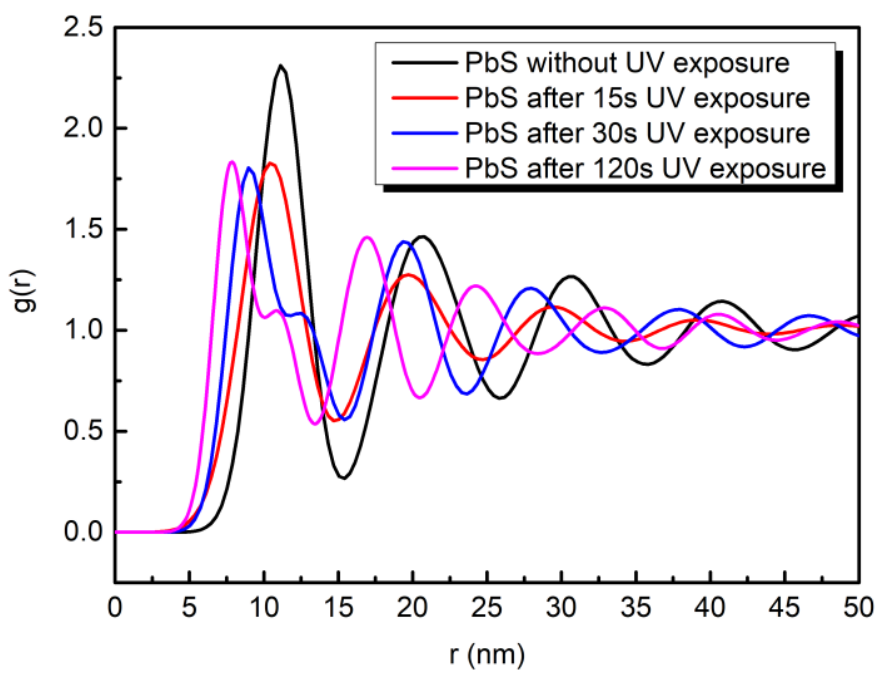

Figure S3. Radial distribution function analysis of TEM images. 


\section{REFERENCE}

1. Markovich, G.; Collier, C. P.; Henrichs, S. E.; Remacle, F.; Levine, R. D.; Heath, J. R., Architectonic Quantum Dot Solids. Accounts of Chemical Research 1999, $32(5), 415-423$.

2. Jun, Y.-w.; Seo, J.-w.; Oh, S. J.; Cheon, J., Recent advances in the shape control of inorganic nano-building blocks. Coordination Chemistry Reviews 2005, 249 (17), 1766-1775.

3. Alivisatos, A. P., Semiconductor Clusters, Nanocrystals, and Quantum Dots. Science 1996, 271 (5251), 933-937.

4. Murray, C. B.; Kagan, C. R.; Bawendi, M. G., Synthesis and Characterization of Monodisperse Nanocrystals and Close-Packed Nanocrystal Assemblies. Annual Review of Materials Science 2000, 30 (1), 545-610.

5. Valizadeh, A.; Mikaeili, H.; Samiei, M.; Farkhani, S. M.; Zarghami, N.; kouhi, M.; Akbarzadeh, A.; Davaran, S., Quantum dots: synthesis, bioapplications, and toxicity. Nanoscale Research Letters 2012, 7 (1), 480.

6. Bera, D.; Qian, L.; Tseng, T.-K.; Holloway, P. H., Quantum Dots and Their Multimodal Applications: A Review. Materials 2010, 3 (4), 2260-2345.

7. Jamieson, T.; Bakhshi, R.; Petrova, D.; Pocock, R.; Imani, M.; Seifalian, A. M., Biological applications of quantum dots. Biomaterials 2007, 28 (31), 4717-4732.

8. Evers, W. H.; De Nijs, B.; Filion, L.; Castillo, S.; Dijkstra, M.; Vanmaekelbergh, D., Entropy-driven formation of binary semiconductor-nanocrystal superlattices. Nano Lett 2010, 10 (10), 4235-41. 
9. Whitham, K.; Smilgies, D.-M.; Hanrath, T., Entropic, Enthalpic, and Kinetic Aspects of Interfacial Nanocrystal Superlattice Assembly and Attachment. Chemistry of Materials 2018, 30 (1), 54-63.

10. Sandeep, C. S. S.; Azpiroz, J. M.; Evers, W. H.; Boehme, S. C.; Moreels, I.; Kinge, S.; Siebbeles, L. D. A.; Infante, I.; Houtepen, A. J., Epitaxially Connected PbSe Quantum-Dot Films: Controlled Neck Formation and Optoelectronic Properties. ACS Nano 2014, 8 (11), 11499-11511.

11. Simon, P.; Bahrig, L.; Baburin, I. A.; Formanek, P.; Röder, F.; Sickmann, J.; Hickey, S. G.; Eychmüller, A.; Lichte, H.; Kniep, R.; Rosseeva, E., Interconnection of Nanoparticles within 2D Superlattices of PbS/Oleic Acid Thin Films. Advanced Materials 2014, 26 (19), 3042-3049.

12. Whitham, K.; Hanrath, T., Formation of Epitaxially Connected Quantum Dot Solids: Nucleation and Coherent Phase Transition. The Journal of Physical Chemistry Letters 2017, 8 (12), 2623-2628.

13. Talapin, D. V.; Lee, J.-S.; Kovalenko, M. V.; Shevchenko, E. V., Prospects of Colloidal Nanocrystals for Electronic and Optoelectronic Applications. Chemical Reviews 2010, 110 (1), 389-458.

14. Boles, M. A.; Engel, M.; Talapin, D. V., Self-Assembly of Colloidal Nanocrystals: From Intricate Structures to Functional Materials. Chemical Reviews 2016, $116(18), 11220-11289$.

15. Cho, K.-S.; Talapin, D. V.; Gaschler, W.; Murray, C. B., Designing PbSe Nanowires and Nanorings through Oriented Attachment of Nanoparticles. Journal of the American Chemical Society 2005, 127 (19), 7140-7147. 
16. Evers, W. H.; Goris, B.; Bals, S.; Casavola, M.; de Graaf, J.; van Roij, R.; Dijkstra, M.; Vanmaekelbergh, D., Low-Dimensional Semiconductor Superlattices Formed by Geometric Control over Nanocrystal Attachment. Nano Letters 2013, 13 (6), 2317-2323.

17. Walravens, W.; De Roo, J.; Drijvers, E.; Ten Brinck, S.; Solano, E.; Dendooven, J.; Detavernier, C.; Infante, I.; Hens, Z., Chemically Triggered Formation of Two-Dimensional Epitaxial Quantum Dot Superlattices. ACS Nano 2016, 10 (7), 6861-6870.

18. Cademartiri, L.; Ghadimi, A.; Ozin, G. A., Nanocrystal Plasma Polymerization: From Colloidal Nanocrystals to Inorganic Architectures. Accounts of Chemical Research 2008, 41 (12), 1820-1830.

19. Ashfold, M. N. R.; Claeyssens, F.; Fuge, G. M.; Henley, S. J., Pulsed laser ablation and deposition of thin films. Chemical Society Reviews 2004, 33 (1), 23-31.

20. Baumgardner, W. J.; Choi, J. J.; Bian, K.; Fitting Kourkoutis, L.; Smilgies, D.M.; Thompson, M. O.; Hanrath, T., Pulsed Laser Annealing of Thin Films of SelfAssembled Nanocrystals. ACS Nano 2011, 5 (9), 7010-7019.

21. Treml, B. E.; Jacobs, A. G.; Bell, R. T.; Thompson, M. O.; Hanrath, T., $\mu-$ Rainbow: CdSe Nanocrystal Photoluminescence Gradients via Laser Spike Annealing for Kinetic Investigations and Tunable Device Design. Nano Letters 2016, 16 (2), $967-$ 972.

22. Whitham, K.; Hanrath, T., On the formation of epitaxially connected quantum dot solids: nucleation and coherent phase transition. Journal of Physical Chemistry Letters 2017, ASAP (12), 2623-2628. 
23. Wang, Y.; Fedin, I.; Zhang, H.; Talapin, D. V., Direct optical lithography of functional inorganic nanomaterials. Science 2017, 357 (6349), 385-388.

24. Ocier, C. R.; Smilgies, D.-M.; Robinson, R. D.; Hanrath, T., Reconfigurable Nanorod Films: An in Situ Study of the Relationship between the Tunable Nanorod Orientation and the Optical Properties of Their Self-Assembled Thin Films. Chemistry of Materials 2015, 27 (7), 2659-2665.

25. Choi, J. J.; Bealing, C. R.; Bian, K.; Hughes, K. J.; Zhang, W.; Smilgies, D.-M.; Hennig, R. G.; Engstrom, J. R.; Hanrath, T., Controlling Nanocrystal Superlattice Symmetry and Shape-Anisotropic Interactions through Variable Ligand Surface Coverage. Journal of the American Chemical Society 2011, 133 (9), 3131-3138.

26. Hanrath, T.; Choi, J. J.; Smilgies, D.-M., Structure/processing relationships of highly ordered lead salt nanocrystal superlattices. ACS nano 2009, 3 (10), 2975-2988.

27. Dong, A. G.; Chen, J.; Vora, P. M.; Kikkawa, J. M.; Murray, C. B., Binary nanocrystal superlattice membranes self-assembled at the liquid-air interface. Nature 2010, 466 (7305), 474-477.

28. Colvin, V. L.; Goldstein, A. N.; Alivisatos, A. P., Semiconductor Nanocrystals Covalently Bound to Metal-Surfaces with Self-Assembled Monolayers. J Am Chem Soc 1992, 114 (13), 5221-5230.

29. Coe-Sullivan, S.; Steckel, J. S.; Woo, W. K.; Bawendi, M. G.; Bulovic, V., Large-area ordered quantum-dot monolayers via phase separation during spin-casting. Adv Funct Mater 2005, 15 (7), 1117-1124. 
30. Zhang, J.; Crisp, R. W.; Gao, J.; Kroupa, D. M.; Beard, M. C.; Luther, J. M., Synthetic Conditions for High-Accuracy Size Control of PbS Quantum Dots. The Journal of Physical Chemistry Letters 2015, 6 (10), 1830-1833.

31. Chen, J.; Song, J. L.; Sun, X. W.; Deng, W. Q.; Jiang, C. Y.; Lei, W.; Huang, J. H.; Liu, R. S., An oleic acid-capped CdSe quantum-dot sensitized solar cell. Applied Physics Letters 2009, 94 (15), 153115.

32. Tarumoto, N.; Miyagawa, N.; Takahara, S.; Yamaoka, T., Diphenyliodonium Salts with Pyranine Conk as an Environment-friendly Photo-acid Generator and Their Applications to Chemically Amplified Resists. 2005, 37 (8), 545-549.

33. Binder, W. H., Supramolecular Assembly of Nanoparticles at Liquid-Liquid Interfaces. Angewandte Chemie International Edition 2005, 44 (33), 5172-5175.

34. Wang, L.; Sahabudeen, H.; Zhang, T.; Dong, R., Liquid-interface-assisted synthesis of covalent-organic and metal-organic two-dimensional crystalline polymers. npj 2D Materials and Applications 2018, 2 (1), 26.

35. Rabani, E.; Reichman, D. R.; Geissler, P. L.; Brus, L. E., Drying-mediated self-assembly of nanoparticles. Nature 2003, 426 (6964), 271-274.

36. Moreels, I.; Lambert, K.; Smeets, D.; De Muynck, D.; Nollet, T.; Martins, J. C.; Vanhaecke, F.; Vantomme, A.; Delerue, C.; Allan, G.; Hens, Z., Size-Dependent Optical Properties of Colloidal PbS Quantum Dots. ACS Nano 2009, 3 (10), 30233030.

37. Whitham, K.; Yang, J.; Savitzky, B. H.; Kourkoutis, L. F.; Wise, F.; Hanrath, T., Charge transport and localization in atomically coherent quantum dot solids. Nature Materials 2016, 15, 557. 
38. Hassinen, A.; Moreels, I.; De Nolf, K.; Smet, P. F.; Martins, J. C.; Hens, Z., Short-Chain Alcohols Strip X-Type Ligands and Quench the Luminescence of PbSe and CdSe Quantum Dots, Acetonitrile Does Not. Journal of the American Chemical Society 2012, 134 (51), 20705-20712.

39. Balazs, D. M.; Dirin, D. N.; Fang, H.-H.; Protesescu, L.; ten Brink, G. H.; Kooi, B. J.; Kovalenko, M. V.; Loi, M. A., Counterion-Mediated Ligand Exchange for PbS Colloidal Quantum Dot Superlattices. ACS Nano 2015, 9 (12), 11951-11959.

40. Zarghami, M. H.; Liu, Y.; Gibbs, M.; Gebremichael, E.; Webster, C.; Law, M., p-Type PbSe and PbS Quantum Dot Solids Prepared with Short-Chain Acids and Diacids. 2010, 4 (4), 2475-2485.

41. Chang, J.; Ogomi, Y.; Ding, C.; Zhang, Y. H.; Toyoda, T.; Hayase, S.; Katayama, K.; Shen, Q., Ligand-dependent exciton dynamics and photovoltaic properties of PbS quantum dot heterojunction solar cells. Phys Chem Chem Phys 2017, $19(9), 6358-6367$.

42. Wellek, R. M.; Mitchell, R. D.; Moore, J. W., Diffusion coefficients of ethylene glycol and cyclohexanol in the solvents ethylene glycol, diethylene glycol, and propylene glycol as a function of temperature. Journal of Chemical \& Engineering Data 1971, 16 (1), 57-60.

43. Moreels, I.; Justo, Y.; De Geyter, B.; Haustraete, K.; Martins, J. C.; Hens, Z., Size-Tunable, Bright, and Stable PbS Quantum Dots: A Surface Chemistry Study. ACS Nano 2011, 5 (3), 2004-2012.

44. Hassinen, A.; Moreels, I.; de Mello Donegá, C.; Martins, J. C.; Hens, Z., Nuclear Magnetic Resonance Spectroscopy Demonstrating Dynamic Stabilization of 
CdSe Quantum Dots by Alkylamines. The Journal of Physical Chemistry Letters 2010, 1 (17), 2577-2581.

45. Liu, Y.; Gibbs, M.; Puthussery, J.; Gaik, S.; Ihly, R.; Hillhouse, H. W.; Law, M., Dependence of carrier mobility on nanocrystal size and ligand length in $\mathrm{PbSe}$ nanocrystal solids. Nano letters 2010, 10 (5), 1960-1969.

46. Nag, A.; Kovalenko, M. V.; Lee, J.-S.; Liu, W.; Spokoyny, B.; Talapin, D. V., Metal-free Inorganic Ligands for Colloidal Nanocrystals: S2-, HS-, Se2-, HSe-, Te2-, HTe-, TeS32-, $\mathrm{OH}-$, and $\mathrm{NH} 2-$ as Surface Ligands. Journal of the American Chemical Society 2011, 133 (27), 10612-10620.

47. Beard, M. C.; Midgett, A. G.; Law, M.; Semonin, O. E.; Ellingson, R. J.; Nozik, A. J., Variations in the Quantum Efficiency of Multiple Exciton Generation for a Series of Chemically Treated PbSe Nanocrystal Films. Nano Letters 2009, 9 (2), 836-845.

48. Urban, J. J.; Talapin, D. V.; Shevchenko, E. V.; Murray, C. B., Self-Assembly of PbTe Quantum Dots into Nanocrystal Superlattices and Glassy Films. Journal of the American Chemical Society 2006, 128 (10), 3248-3255.

49. Grisorio, R.; Debellis, D.; Suranna, G. P.; Gigli, G.; Giansante, C., The Dynamic Organic/Inorganic Interface of Colloidal PbS Quantum Dots. Angewandte Chemie International Edition 2016, 55 (23), 6628-6633.

50. Grisorio, R.; Debellis, D.; Suranna, G. P.; Gigli, G.; Giansante, C., The Dynamic Organic/Inorganic Interface of Colloidal PbS Quantum Dots. Angew. Chem., Int. Ed. 2016, 55 (23), 6628-33. 
51. Bealing, C. R.; Baumgardner, W. J.; Choi, J. J.; Hanrath, T.; Hennig, R. G., Predicting Nanocrystal Shape through Consideration of Surface-Ligand Interactions. ACS Nano 2012, 6 (3), 2118-2127.

52. Abelson, A.; Qian, C.; Salk, T.; Luan, Z.; Fu, K.; Zheng, J.-G.; Wardini, J. L.; Law, M., Collective topo-epitaxy in the self-assembly of a 3D quantum dot superlattice. Nature Materials 2020, 19 (1), 49-55.

53. Whitham, K.; Yang, J.; Savitzky, B. H.; Kourkoutis, L. F.; Wise, F.; Hanrath, T., Charge transport and localization in atomically coherent quantum dot solids. Nat Mater 2016, 15 (5), 557-63.

54. Baumgardner, W. J.; Whitham, K.; Hanrath, T., Confined-but-Connected Quantum Solids via Controlled Ligand Displacement. Nano Letters 2013, 13 (7), 3225-3231.

55. Geuchies, J. J.; van Overbeek, C.; Evers, W. H.; Goris, B.; de Backer, A.; Gantapara, A. P.; Rabouw, F. T.; Hilhorst, J.; Peters, J. L.; Konovalov, O.; Petukhov, A. V.; Dijkstra, M.; Siebbeles, L. D. A.; van Aert, S.; Bals, S.; Vanmaekelbergh, D., In situ study of the formation mechanism of two-dimensional superlattices from PbSe nanocrystals. Nature Materials 2016, 15, 1248.

56. van Huis, M. A.; Kunneman, L. T.; Overgaag, K.; Xu, Q.; Pandraud, G.; Zandbergen, H. W.; Vanmaekelbergh, D., Low-Temperature Nanocrystal Unification through Rotations and Relaxations Probed by in Situ Transmission Electron Microscopy. Nano Letters 2008, 8 (11), 3959-3963. 
57. Abelson, A.; Qian, C.; Salk, T.; Luan, Z.; Fu, K.; Zheng, J.-G.; Wardini, J. L.; Law, M., Collective topo-epitaxy in the self-assembly of a 3D quantum dot superlattice. Nat. Mater. 2019, 1-7.

58. Whitham, K., Structure and Electronic Properties of Lead Selenide Nanocrystal Solids. 2016.

59. Grisorio, R.; Quarta, D.; Fiore, A.; Carbone, L.; Suranna, G. P.; Giansante, C., The dynamic surface chemistry of colloidal metal chalcogenide quantum dots. Nanoscale Advances 2019, 1 (9), 3639-3646.

60. Vaz, R. V.; Gomes, J. R. B.; Silva, C. M., Molecular dynamics simulation of diffusion coefficients and structural properties of ketones in supercritical CO 2 at infinite dilution. The Journal of Supercritical Fluids 2016, 107, 630-638.

61. Kagan, C. R.; Murray, C. B., Charge transport in strongly coupled quantum dot solids. Nature Nanotechnology 2015, 10, 1013.

62. Wang, Y.; Pan, J.-A.; Wu, H.; Talapin, D. V., Direct Wavelength-Selective Optical and Electron-Beam Lithography of Functional Inorganic Nanomaterials. ACS Nano 2019.

63. Zhao, S.; Caruso, F.; Dähne, L.; Decher, G.; De Geest, B. G.; Fan, J.; Feliu, N.; Gogotsi, Y.; Hammond, P. T.; Hersam, M. C.; Khademhosseini, A.; Kotov, N.; Leporatti, S.; Li, Y.; Lisdat, F.; Liz-Marzán, L. M.; Moya, S.; Mulvaney, P.; Rogach, A. L.; Roy, S.; Shchukin, D. G.; Skirtach, A. G.; Stevens, M. M.; Sukhorukov, G. B.; Weiss, P. S.; Yue, Z.; Zhu, D.; Parak, W. J., The Future of Layer-by-Layer Assembly: A Tribute to ACS Nano Associate Editor Helmuth Möhwald. ACS Nano 2019, 13 (6), 6151-6169. 
64. Zhang, R.; Shang, J.; Xin, J.; Xie, B.; Li, Y.; Möhwald, H., Self-assemblies of luminescent rare earth compounds in capsules and multilayers. Advances in Colloid and Interface Science 2014, 207, 361-375.

65. Skorb, E. V.; Möhwald, H., "Smart” Surface Capsules for Delivery Devices. Advanced Materials Interfaces 2014, 1 (6), 1400237.

66. Vyatskikh, A.; Delalande, S.; Kudo, A.; Zhang, X.; Portela, C. M.; Greer, J. R., Additive manufacturing of 3D nano-architected metals. Nature Communications 2018, $9(1), 593$.

67. Yee, D. W.; Lifson, M. L.; Edwards, B. W.; Greer, J. R., Additive Manufacturing of 3D-Architected Multifunctional Metal Oxides. Advanced Materials 2019, 31 (33), 1901345. 